Illinois State University

ISU ReD: Research and eData

Theses and Dissertations

$6-21-2021$

\title{
Characterizing Self-Inserting Carbon-Fiber Microelectrode Arrays for Fast-Scan Cyclic Voltammetry
}

Brad M. Smith

Illinois State University, bmsmit3@gmail.com

Follow this and additional works at: https://ir.library.illinoisstate.edu/etd

\section{Recommended Citation}

Smith, Brad M., "Characterizing Self-Inserting Carbon-Fiber Microelectrode Arrays for Fast-Scan Cyclic Voltammetry" (2021). Theses and Dissertations. 1470.

https://ir.library.illinoisstate.edu/etd/1470

This Thesis is brought to you for free and open access by ISU ReD: Research and eData. It has been accepted for inclusion in Theses and Dissertations by an authorized administrator of ISU ReD: Research and eData. For more information, please contact ISUReD@ilstu.edu. 


\section{CHARACTERIZING SELF-INSERTING CARBON-FIBER MICROELECTRODE ARRAYS FOR FAST-SCAN CYCLIC VOLTAMMETRY}

\section{BRAD M. SMITH}

\section{Pages}

The ability to monitor brain neurotransmitters with high fidelity is critical for establishing the neural underpinnings of behavior and pathology. Fast-scan cyclic voltammetry (FSCV) is an electroanalytical technique that relies on sweeping electrical potentials to drive rapid oxidation and reduction of electroactive neurochemicals. FSCV applications are typically limited by their ability to measure from one to two recording sites using a well-established microsensor, the carbon-fiber microelectrode (CFM). When coupled to FSCV, the CFM provides exquisite spatial, temporal, and chemical resolution in vivo. Recent advances have led to the development of self-inserting (i.e., without the requirement for a shuttle) carbon-fiber microelectrode arrays (CF-MEAs) capable of measuring up to sixteen sites simultaneously. Here we characterize CF-MEAs for measuring dopamine (DA), a neurotransmitter critical for locomotion, motivation, and cognition that also plays a substantive role in the pathologies of Parkinson's disease, substance use disorder, and schizophrenia. In general, performance of CF-MEAs for FSCV DA monitoring assessed with flow injection analysis, a "beaker" technique that directs a laminar bolus of dopamine to the sensing surface, compared favorably with those of the conventional CFM. Measurements in the urethaneanesthetized rat also suggest that CF-MEAs are capable of capturing heterogeneous DA signals in the striatum, a brain region densely innervated by DA neurons and heavily implicated in reward learning, with high fidelity to conventional CFMs but simultaneously at multiple sites across a 
much broader measurement field. We conclude that CF-MEAs perform favorably in comparison to CFMs with regard to temporal response, DA sensitivity, background signal, signal-to-noise ratio, and peak oxidative potential. Moreover, CF-MEAs have a distinct advantage over CFMs to characterize the heterogeneous nature of DA signaling in vivo through their ability to simultaneously monitor a $1.5 \mathrm{~mm}$ mediolateral span of the brain in $100-\mu \mathrm{m}$ increments.

KEYWORDS: carbon fiber microelectrode (CFM); carbon fiber microelectrode array (CF-MEA); flow injection analysis (FIA); dopamine (DA); fast-scan cyclic voltammetry (FSCV); amphetamine (AMPH) 


\title{
CHARACTERIZING SELF-INSERTING CARBON-FIBER MICROELECTRODE ARRAYS \\ FOR FAST-SCAN CYCLIC VOLTAMMETRY
}

BRAD M. SMITH

\author{
A Thesis Submitted in Partial \\ Fulfillment of the Requirements \\ for the Degree of \\ MASTER OF SCIENCE \\ School of Biological Sciences \\ ILLINOIS STATE UNIVERSITY
}

2021 
(C) 2021 Brad M. Smith 


\section{CHARACTERIZING SELF-INSERTING CARBON-FIBER MICROELECTRODE ARRAYS FOR FAST-SCAN CYCLIC VOLTAMMETRY}

BRAD M. SMITH

COMMITTEE MEMBERS:

Paul A. Garris, Chair

Joseph M. Casto

Wolfgang Stein 


\section{ACKNOWLEDGMENTS}

I would like to offer my heartfelt gratitude to Dr. Paul A. Garris for taking a chance on a non-traditional student that had been absent from science for nearly a decade. The level of compassion, dedication, and expertise you have shown is second to none. You have been beyond supportive of my academic and professional aspirations, and for that I am grateful. I would also like to thank Dr. Joseph Casto and Dr. Wolfgang Stein for their support and guidance as both faculty and members of my committee. Finally, I would like to acknowledge Dr. Cynthia Chestek, Dr. Paras Patel, and Julianna Richie at University of Michigan for the outstanding collaboration over the past two years.

To Mom, Dad, Amy, Tom, Kim, and Seth: your unconditional love and support mean the world to me, and none of this would have been possible without you. Maybe now I'll finally be done with school. Then again, maybe not.

B.M.S. 


\section{CONTENTS}

Page

ACKNOWLEDGMENTS

CHAPTER I: INTRODUCTION 1

$\begin{array}{ll}\text { CHAPTER II: METHODS } & 8\end{array}$

$\begin{array}{ll}\text { Animals } & 8\end{array}$

$\begin{array}{ll}\text { Sensors } & 8\end{array}$

Fast-scan Cyclic Voltammetry 9

Flow Injection Analysis $\quad 9$

$\begin{array}{ll}\text { Surgery } & 10\end{array}$

Data Analysis $\quad 11$

$\begin{array}{ll}\text { Statistics } & 12\end{array}$

$\begin{array}{ll}\text { Chemicals } & 13\end{array}$

$\begin{array}{ll}\text { Drugs } & 13\end{array}$

CHAPTER III: RESULTS - CHARACTERIZATION OF CF-MEAs WITH FLOW

$\begin{array}{ll}\text { INJECTION ANALYSIS } & 14\end{array}$

$\begin{array}{ll}\text { Sensor Positioning } & 14\end{array}$

Performance Metrics 16

Temporal Response $\quad 16$

$\begin{array}{ll}\text { Sensitivity } & 19\end{array}$

Peak Oxidative Potential of Dopamine $\quad 20$

$\begin{array}{ll}\text { Root-Mean-Square Noise } & 21\end{array}$

$\begin{array}{ll}\text { Total Background Current } & 22\end{array}$ 
Signal-to-Noise Ratio

Principle Component Regression

CHAPTER IV: RESULTS - IN VIVO CHARACTERIZATION OF CF-MEAs

Background

CF-MEAs Capture Heterogeneity of Electrically Evoked Dopamine Signals

In Vivo CF-MEA Characteristics

CF-MEAs Capture Effects of AMPH on Baseline and Phasic Dopamine Signals

CHAPTER V: CONCLUSION

REFERENCES

APPENDIX: FIGURES 


\section{CHAPTER I: INTRODUCTION}

Monitoring neurotransmitters in the brain is crucial for establishing the neural underpinnings of behavior and pathology. Although several techniques have been developed for this purpose, microdialysis and voltammetry have arguably dominated the modern era of neurochemical measurements (Watson et al., 2006; Robinson et al., 2008; Sandberg and Garris, 2010). In microdialysis, a sample of extracellular fluid is physically removed by the probe for external analysis with techniques such as high-performance liquid chromatography coupled to electrochemical detection or gas chromatography-mass spectrometry, whereas analytes are electrochemically measured directly at the implanted microsensor in situ in voltammetry. A radically different analytical approach, genetically encoded indicators, has been more recently developed and received considerable attention for its potential to further advance neurochemical measurements (Lin and Schnitzer, 2016; Patriarchi et al., 2019; Jing et al., 2019; Sabatini and Tiam, 2020; Yocky and Covey, 2020). In this scheme, analyte binding to a brain cell-expressed indicator (e.g., based on a neurotransmitter receptor) alters fluorescence that is detected by fiber photometry, one-photon microendoscopy, or two-photon microscopy.

Fast-scan cyclic voltammetry (FSCV) at a carbon-fiber microelectrode (CFM) has long been considered the gold standard for real-time neurochemical measurements at a microsensor (Venton and Cao, 2020; Puthongkham and Venton, 2020). Overall, FSCV at a CFM is noted for its high temporal, chemical, and spatial resolution. Temporal resolution is provided by application of a voltage scan every $100 \mathrm{~ms}$, during which an analyte measurement occurs. Chemical resolution is provided by the voltammogram, a profile of measured current versus applied that functions as a chemical signature, collected during each scan. The CFM is also an excellent sensor for FSCV. Its carbon surface is well suited for performing electrochemistry in the brain (Robinson et al., 2008), 
and its size $(\mathrm{r}=3.5 \mu \mathrm{m})$ provides cell-approximate spatial resolution with minimal tissue trauma during implantation (Jaquins-Gerstl and Michael, 2015).

Another attractive analytical characteristic of FSCV at a CFM is versatility. Although most work to date has focused on dopamine (DA), a neurotransmitter involved in motivation, movement, and cognition (Schultz 2007), in the rat striatum, there have been exciting developments establishing FSCV at a CFM for: (1) non-DA neurotransmitters (e.g., serotonin, norepinephrine, epinephrine, histamine, octopamine, adenosine, nitric oxide, and neuropeptides), as well as a variety of neurochemicals (e.g., hydrogen peroxide, ascorbic acid, uric acid, oxygen, and $\mathrm{pH}$ ); (2) non-striatal brain regions (e.g., amygdala, cortex, raphe nucleus, bed nucleus of the stria terminalis, thalamus, substantia nigra pars reticulata and compacta, ventral tegmental area, hippocampus, spinal cord, and median eminence); (3) non-rat animal models (e.g., European starling, zebra finch, mouse, vole, Syrian hamster, guinea pig, monkey, human, lamprey, zebrafish, fruit fly, and honey bee) (Garris et al., 1994; Rice et al., 1994; Gale and Perkel, 2006; Greco et al., 2006; Robinson et al., 2008; Park and West, 2009; Huffman and Venton, 2009; Hashemi et al., 2011; Chang et al., 2012; Glanowska et al., 2012; Fortin et al., 2015; Smith et al., 2015; Pyakurel et al., 2016; Srejic et al., 2015; Resendez et al., 2016; Shin et al., 2017; Noga et al., 2017; Jarriault et al., 2018; Roberts and Sombers, 2018; Saylor et al., 2019; Stagkourakis et al., 2019; Tan et al., 2021). Versatility is also conferred because FSCV does not require the transgenic manipulation (Patriarchi et al., 2018; Sun et al., 2018) or contrast agent infusions needed for other neurochemical sensing techniques (Li and Hasanoff, 2020).

Because of different measurement strategies, microdialysis, FSCV at a CFM, and genetically encoded indicators exhibit different analytical characteristics (Wang et al., 2018). In general, microdialysis exhibits superior sensitivity and chemical resolution while FSCV at a CFM 
excels at temporal and spatial resolution. Two critical technical advancements have reduced the performance gap between FSCV at a CFM and microdialysis. Sensitivity of FSCV has been increased by extending the potential limits of the voltage scan to overoxidize the carbon surface of the CFM and increase analyte adsorption (Heien et al., 2003). Chemical resolving power of FSCV has been enhanced with a chemometrics approach called principal component regression (PCR) (Heien et al., 2005; Keithley et al., 2009). These improvements have led to the detection of behaviorally relevant sub-second to second DA signals called transients (Day et al., 2007) and more slowly occurring alterations in basal DA levels (Howe et al., 2013) measured in units of concentration change relative to a zeroed background. Moreover, absolute concentrations of basal DA have now been measured using a CFM with alternative waveforms such as fast-scan adsorption-controlled voltammetry (Atcherley et al., 2013, 2015) and fast-cyclic square-wave voltammetry (Park et al., 2018; Oh et al., 2018).

Although direct quantitative comparisons are not yet available, the DA-receptor based indicators of $\mathrm{GRAB}_{\mathrm{DA}}$ and dLight (Patriarchi et al., 2018; Sun et al., 2018) appear to exhibit superior temporal and chemical resolution and sensitivity for DA measurements compared to FSCV at a CFM. Comparisons of spatial resolution depend upon the scheme for fluorescent detection. For example, the probe in fiber photometry is considerably larger than the CFM (>100 $\mu \mathrm{m}$ versus $8 \mu \mathrm{m}$ in diameter, respectively). On the other hand, 2-photon microscopy can detect DA release from a single DA fiber in a mouse striatal slice (Sun et al., 2018) and within 17- $\mu \mathrm{m}^{2}$ regions of interest in the cortex of an awake, head-fixed mouse (Patriarchi et al., 2018). Activity of single DA neurons is also detected using the $\mathrm{Ca}^{2+}$ indicator GCaMP with 1-photon microenodoscopy in a freely moving mouse, but a large $(\approx 500 \mathrm{um})$ gradient index lens must be implanted above the target region (da Silva et al., 2018). An exciting application of this approach 
was monitoring the activity of the same DA neuron across days. Thus, while powerful, the enhanced spatial resolution of genetically encoded indicators and the ability for longitudinal measurements of the same neuron come with some restrictions. In contrast to FSCV at a CFM, which can be calibrated pre- or post-experiment and even in situ (Schuweiler et al., 2018), GRAB $_{\mathrm{DA}}$ and dLight measurements are not easily converted to concentration. Other limitations of genetically encoded indicators are photobleaching, avoidance of pharmacological agents acting at the DA receptor, and the requirement that the animal model is amenable to transgenic modification. Both genetically encoded indicators and FSCV at a CFM exhibit a similar, somewhat restricted capability for simultaneous multianalyte monitoring, which is considerably less than that for microdialysis.

A CFM acutely implanted with a detachable micromanipulator was initially used for FSCV measurements of DA in the freely moving rat (Garris et al., 1997). The subsequent development of a chronically implantable CFM permitted longitudinal measurements at the same recording site over months (Clark et al., 2010). Despite the profound impact of these technical advances on investigations of DA-behavior relationships (e.g., Phillips et al., 2003; Day et al., 2007; Gan et al., 2010; Flagel et al., 2011), the number of recording sites in a single animal was typically limited to one or two. By contrast, fiber photometry with arrays (Guo et al., 2015) or distributed probes (Kim et al., 2016) and subdivision of the viewing field into regions of interest in 2-photon microscopy (Patriarchi et al., 2018; Sun et al., 2018) affords simultaneous neurochemical interrogation of multiple sites. Guo et al. (2015) have performed three channel fiber photometry with success, but the large diameter $(\sim 200 \mu \mathrm{m})$ of optical fibers makes recording with high spatial resolution challenging. 
The well-established use of FSCV at a CFM has led to the development of multi-site arrays that take advantage of the temporal and spatial resolving power of FSCV. While several multi-site recording devices have been developed and tested, each has physical properties or technical challenges that reduce their utility. One such example is a planar probe that employs pyrolyzed photoresist electrodes (PPEs) (Zachek et al., 2009). While performance of PPEs was favorable in in vitro characterization, relatively slow response time to DA as well as the overall size ( $\sim 200 \mu \mathrm{m})$ suggests that further work is needed prior to routine in vivo utilization. Carbon fiber multielectrode arrays (CF-MEAs) have been in development for over a decade, with multiple designs performing favorably during characterization. Initial designs were utilized for electrophysiology applications in the cortex (Patel et al., 2015). While this application required fiber lengths much shorter than are necessary for midbrain measurements, the fragility of carbon fibers necessitated use of structural support to facilitate insertion into the cortex. One way demonstrated to support CF-MEA implantation was a single-width $(\approx 30 \times 24 \mu \mathrm{m})$, beveled-tip silicon support structure that was successful for acute cortical electrophysiological recording. Alternatively, a biodissolvable polyethylene glycol (PEG)-based substrate was used to facilitate fiber insertion while maintaining the small diameter of the carbon fiber and supported chronic cortical electrophysiology recording. This PEG-based insertion shuttle has applications for both FSCV and electrophysiological measurements. Indeed, the use of PEG-based substrates has been used successfully on CF-MEAs capable of FSCV measurements in the midbrain (Schwerdt et al., 2017; Schwerdt et al., 2018), but dissolution of PEG can present technical challenges that complicate insertion. To overcome the challenges associated with PEG-based substrates, engineers at University of Michigan developed rectangular $(\sim 1.3 \mathrm{~mm}$ x $0.2 \mathrm{~mm})$ guide cannulas for implantation to protect CF-MEAs during placement (Patel et al., 2020). While the guide cannula 
is highly effective in facilitating fiber insertion, its size leads to a significant level of tissue disruption during insertion.

Here, in an effort to minimize tissue trauma caused by guide cannula insertion and to avoid complications with PEG dissolution, we assess the performance of newly developed siliconsupported CF-MEAs for the purpose of obtaining DA measurements using FSCV in the striatum, a deep subcortical structure. The silicon support structure is tapered from 55.5 to $15.5 \mu \mathrm{m}$ (thickness of $\sim 20 \mu \mathrm{m}$ ), leaving $500 \mu \mathrm{m}$ of only carbon fiber beyond the tip. The newly developed CF-MEA was compared to the performance of the conventional CFM. Flow injection analysis (FIA) was used to assess sensor temporal response, sensitivity, root-mean-square (RMS) noise, total background current (TBC), and signal-to-noise ratio (SNR). Follow-up measurements using a urethane-anesthetized rat model assessed the ability of CF-MEAs to monitor DA in the striatum in vivo. DA signals, consisting of electrically evoked transient-like signals, changes in baseline DA concentration, and phasic DA transients, were used to assess CF-MEA performance. The conventional CFM has been widely used in brain slices and in vivo to monitor electrically, optically, pharmacologically, and behaviorally-evoked DA signals for studies focusing on DA neuroscience and pharmacology (Robinson et al., 2008; Sandberg and Garris, 2010; Puthongkham and Venton, 2020). Thus, performance was not only characterized in terms of consistency between sites of CF-MEAs and between CF-MEAs of the same type but also in terms of differences between CF-MEAs and CFMs. Based on our analysis of in vitro and in vivo data, we conclude that silicon-supported CF-MEAs perform favorably in comparison to CFMs with regard to temporal response, DA sensitivity, background signal, SNR, and peak oxidative potential of DA. Moreover, silicon-supported CF-MEAs are shown to have a distinct advantage over CFMs to characterize the 
heterogeneous nature of DA signaling in the striatum in vivo through their ability to simultaneously monitor a $1.5 \mathrm{~mm}$ mediolateral span of the dorsal striatum in $100-\mu \mathrm{m}$ increments. 


\title{
CHAPTER II: METHODS
}

\begin{abstract}
Animals
Adult male Sprague-Dawley rats (300-350g) were purchased from Envigo (Indianapolis, IN, USA) and housed in the temperature-controlled Felmley Science Annex with a 12-hour light/dark cycle. Food and water was provided ad libitum. All procedures were carried out in accordance with protocols approved by the Illinois State University Institutional Animal Care and
\end{abstract} Use Committee (IACUC).

\section{Sensors}

CFMs (Fig. 1A, far left) were constructed by aspirating an individual carbon fiber $(7-\mu \mathrm{m}$ diameter, Cytec Engineering Materials, West Patterson, NJ, USA) into a borosilicate capillary tube (1.2 mm outer diameter, $0.8 \mathrm{~mm}$ inner diameter, Sutter Instrument, Novato, CA, USA) and pulled using a vertical micropipette puller (Narishige, Tokyo, Japan). After separation, the distal end of the capillary tube was fractured to create an opening of $\sim 20 \mu \mathrm{m}$ in diameter. The capillary was sealed with epoxy (Miller-Stephenson Chemical, Morton Grove, IL USA) and allowed to cure at room temperature for 72 hours. The carbon fiber was then trimmed to a length of $50 \mu \mathrm{m}$ beyond the epoxy seal, and electrical connection between the carbon fiber and lead wire was established by inserting and melting a small piece of bismuth alloy (Small Parts, Inc., Miramar, FL, USA) with a heat gun (Master Appliance Corp., Racine, WI, USA).

Both 8-site and 16-site CF-MEAs (Fig. 1A) were provided by the Chestek Lab (University of Michigan, Ann Arbor, MI, USA). The CF-MEAs used in this study are custom fabricated selfinsertable devices designed to measure both electrophysiological and neurochemical signals. To briefly summarize the manufacturing process, printed circuit boards are manufactured with either 8 or 16 traces/trenches to accommodate electrical connection of individual carbon fibers. 
Individual carbon fibers are coated with the insulation Parylene $\mathrm{C}$ and reinforced by a silicon shank that decreases in diameter stepwise toward the exposed carbon-fiber tip (Fig. 1A INSETS). Laser ablation of the Parylene $\mathrm{C}$ re-exposes and increases the surface area of $50 \mu \mathrm{m}$ of carbon fiber, the same length of fiber exposed on traditional CFMs, and oxygen plasma ashing is performed to maximize surface area. Additional CF-MEA fabrication details and software specifications can be found in Patel et al. (2015) and Patel et al. (2020).

\section{Fast-Scan Cyclic Voltammetry}

FSCV is recorded by scanning a triangular waveform $(400 \mathrm{~V} / \mathrm{s})$ from $-0.4 \mathrm{~V}$ to $+1.3 \mathrm{~V}$ at a sampling rate of $10 \mathrm{~Hz}$. The sensing surface is held at $-0.4 \mathrm{~V}$ between scans. Applied potentials are referenced to an $\mathrm{Ag} / \mathrm{AgCl}$ electrode, constructed from chloridizing the exposed tip of a Tefloncoated silver wire (Stoelting, Wood Dale, IL, USA). Both CFMs and CF-MEAs are connected to a headstage (Fig. 1A), which is routed via a lead cable (Fig. 1B) to a breakout box (Fig. 1C). The breakout box interfaces between the headstage and two PCIe-7841 data acquisition boards (National Instruments, Austin, TX, USA) installed in a PC computer and is powered by a standalone power supply (BK Precision, Yorba Linda, CA, USA; Fig. 1D). All data are collected using the LabView-based (National Instruments, Austin, TX, USA) Wolverine FSCV program (Chestek Lab, University of Michigan, Ann Arbor, MI, USA) and analyzed using HDCV (University of North Carolina, Chapel Hill, NC, USA).

\section{Flow Injection Analysis}

The FIA flow cell (Fig. 2) (Rheodyne, Rohnert Park, CA, USA) operates using a nitrogendriven pneumatic actuator that is triggered by a user-defined transistor-transistor logic (TTL) square wave pulse generated in the Wolverine FSCV data acquisition program (Chestek Lab, University of Michigan, Ann Arbor, MI, USA). Tris buffer is continuously pumped into a custom- 
built flow cell reservoir using a syringe pump (Harvard Apparatus, Cambridge, MA, USA) at a rate of $240 \mathrm{~mL} / \mathrm{min}$. DA solution is pre-loaded into an injection loop and is delivered as a bolus in the flow stream during a user-defined period when triggered by the TTL pulse. The bolus of known-concentration analyte allows for extensive analysis of performance of both CFMs and CFMEAs.

\section{Surgery}

Rats were anesthetized using urethane (Acros Organics, Geel, Belgium) dissolved in 0.9\% (150 mM) normal saline (1.6 g/kg i.p.) and subsequently immobilized in a stereotaxic frame (David Kopf Instruments, Tujunga, CA, USA). A linear incision was made in the scalp to expose the skull, and burr holes were drilled with a high-speed rotary tool (Foredom, Bethel, CT, USA) based on anatomical reference points (Paxinos and Watson, 1986) to accommodate placement of the stimulating electrode (AP -4.6, ML +1.3, DV -8.0 relative to bregma), reference electrode (contralateral superficial cortex), and sensors $(\mathrm{AP}+1.2, \mathrm{ML}+1.5, \mathrm{DV}-4.0$ and $\mathrm{AP}+1.2, \mathrm{ML}+3.0$, DV -4.0, both relative to bregma). Computer-generated electrical stimulation was passed through an optical isolator and constant-current generator (Digitimer Limited, Letchworth Garden City, United Kingdom). Stimulation pulses were applied to a twisted bipolar electrode (Plastics One, Roanoke, VA, USA), with tips polished and separated by $1 \mathrm{~mm}$. Dorsoventral depth of the stimulating electrode optimized atop the medial forebrain bundle until robust electrically evoked DA release was observed at a single CFM. Stimulus trains consisted of biphasic pulses (2 ms for each phase) delivered at an intensity of $\pm 300 \mu \mathrm{A}$, a frequency of $60 \mathrm{~Hz}$, and a duration ranging from $0.4 \mathrm{~s}$ ( 24 pulses) to $3 \mathrm{~s}$ (180 pulses). Stimulus parameters were selected to elicit DA signals comparable in dynamics and amplitude to DA transients (Robinson and Wightman, 2007). In 
experiments requiring intravenous (i.v.) administration of drugs, venous access was established via cutdown and direct visualization of the femoral vein to ensure i.v. patency.

\section{Data Analysis}

Cyclic voltammograms were filtered in HDCV using a lowpass Bessel setting. Temporal response of sensors was recorded as the amount of time from baseline to $85 \%$ of normalized maximal current observed during FIA injections. Sensitivity (nA/ $\mu \mathrm{M}$ DA) was determined by exposing sensors to DA boluses at varying concentrations and calculating the resulting slope of a linear regression curve. Root-mean-square (RMS) noise was calculated using current tracings taken at the peak oxidative potential for DA in ten consecutive measurements at the beginning of FSCV recordings. Total background current (TBC) was calculated by summing the absolute value current of all 1,000 data points in the cyclic voltammogram at the beginning of FSCV recordings. Finally, signal-to-noise ratio (SNR) was calculated by dividing signal maximal current by RMS noise.

Background subtraction for in vivo electrically evoked transient-like signal analysis occurred by averaging ten cyclic voltammograms at the beginning of recordings. Background subtraction for in vivo non-evoked signal analysis occurred by averaging ten cyclic voltammograms immediately prior to the observed event. Training set data was captured in HDCV, and principle component regression (PCR), a chemometrics approach combining principle component analysis and inverse least-squares regression, was utilized to quantify changes in DA concentration and isolate the effects of change in $\mathrm{pH}$ and baseline drift. Principle component regression output was considered valid when $\mathrm{Q}$ scores did not exceed $\mathrm{Q}_{\alpha}$ as calculated by HDCV. Hangup, defined as adsorption of DA to the sensing surface, was removed and data were fit to the 3-parameter restricted diffusion model of Michael and co-workers (Walters et al., 2015): 


$$
\begin{aligned}
& \frac{\mathrm{dDA}_{\mathrm{ic}}}{\mathrm{d} t}=R_{\mathrm{p}} f-\mathrm{DA}_{\mathrm{ic}} k_{\mathrm{T}} \\
& \frac{\mathrm{d}[\mathrm{DA}]_{\mathrm{oc}}}{\mathrm{d} t}=\frac{\mathrm{DA}_{\mathrm{ic}} k_{\mathrm{T}}}{V_{\mathrm{oc}}}-[\mathrm{DA}]_{\mathrm{oc}} k_{\mathrm{U}}
\end{aligned}
$$

where $\mathrm{DA}_{\mathrm{ic}}$ is the amount of DA (in moles) in the inner compartment, $R_{\mathrm{p}}$ is the moles of DA released per stimulus pulse, $f$ is the stimulus frequency, $k_{\mathrm{T}}$ is a first-order rate constant for DA transport from inner to outer compartment, $V_{\mathrm{oc}}$ is the volume of the outer compartment, $k_{\mathrm{U}}$ is the first-order rate constant for DA uptake, and $[\mathrm{DA}]_{\mathrm{oc}}$ is the concentration of DA in the outer compartment. The simulated response was calculated from fitted parameters for $R_{\mathrm{p}}, k_{\mathrm{T}}$, and $k_{\mathrm{U}}$. Non-evoked DA signals were analyzed using MiniAnalysis (Synaptosoft, Decatur, GA, USA). Non-evoked signals with a SNR of greater than five times RMS noise were identified as DA transients.

Evoked signal types were identified in the method of Taylor et al. (2015). These electrically evoked signals (180 p, $60 \mathrm{~Hz}, \pm 300 \mu \mathrm{A})$ include both fast (types 1, 2, 3, and 4) and slow responses. Type I signals increase in a linear duration for the entire duration of the 3-s stimulation, and type II signals increase for the duration of the 3-s stimulation but do so in a non-linear fashion. Type III signals (not observed in this study) reach a maximal change in current prior to the end of the stimulation, and type IV signals increase in a biphasic fashion for the entire duration of the stimulation. A signal was considered a slow response if it failed to reach detection limit within 200 ms of the onset of the stimulation.

\section{Statistics}

FIA data was collected in replicates of three. Data was analyzed using one-way nested ANOVA (CF-MEA-8s and CF-MEA-16s) and one-way ANOVA (CFMs and between sensor 
type) to examine the characteristics both within and between sensor types. Post-hoc TukeyKramer analysis allowed for determination of which sites or sensors differed significantly. Fidelity of PCR-resolved FIA data was assessed using a Pearson correlation coefficient. In vivo kinetic parameters pre- and post-drug were compared with a paired t-test. All statistical tests utilized a significance value of $\mathrm{p}=0.05$.

\section{Chemicals}

To determine analyte response characteristics via FIA, a 5-mM DA stock solution was created by dissolving DA hydrochloride (Sigma-Aldrich, St. Louis, MO, USA) in a $0.15 \mathrm{M}$ perchloric acid solution. The resulting solution was protected from light and stored at $4^{\circ} \mathrm{C}$ until and between use. A modified Tris buffer was utilized during all FIA measurements and consisted of $15 \mathrm{mM}$ Tris, $140 \mathrm{mM} \mathrm{NaCl}, 3.25 \mathrm{mM} \mathrm{KCl}, 1.2 \mathrm{mM} \mathrm{CaCl}_{2}, 1.25 \mathrm{mM} \mathrm{NaH}_{2} \mathrm{PO}_{4}, 1.2 \mathrm{mM} \mathrm{MgCl}_{2}$, and $2.0 \mathrm{mM} \mathrm{Na}_{2} \mathrm{SO}_{4}$ in nanopure water brought to a physiologic $\mathrm{pH}$ of 7.4 with a small quantity of $\mathrm{NaOH}$. The Tris buffer was stored at $4^{\circ} \mathrm{C}$ when not in use and was brought to room temperature prior to data collection. $0.25-, 0.5-, 0.75-$, and $1.0-\mu \mathrm{M}$ DA solutions were created by micropipetting aliquots of $5 \mathrm{mM}$ DA stock solution into $50 \mathrm{~mL}$ of prepared Tris buffer measured in a volumetric flask. $\mathrm{pH} 6.9,7.15,7.65$, and 7.90 buffer was made by adding small quantities of hydrochloric acid or sodium hydroxide. DA-containing solutions used for analysis were protected from light and replaced every 30 minutes to mitigate the effects of DA degradation at room temperature.

\section{Drugs}

D-amphetamine hemisulfate (Sigma-Aldrich, St. Louis, MO, USA) was dissolved in 0.9\% normal saline and administered via i.v. $(1 \mathrm{mg} / \mathrm{kg})$ or i.p $(10 \mathrm{mg} / \mathrm{kg})$. Raclopride tartrate (Sigma-Aldrich, St. Louis, MO, USA) was administered $2 \mathrm{mg} / \mathrm{kg}$ i.p in a cocktail with D-amphetamine hemisulfate. 


\section{CHAPTER III: RESULTS - CHARACTERIZATION OF CF-MEAs WITH FLOW INJECTION}

ANALYSIS

\section{Sensor Positioning}

In conventional FIA, a CFM is centered in a flowing stream of buffer at the interface between the tubing outlet and buffer reservoir of the flow cell to record the fastest response to the analyte bolus (Fig. 3, top right). The initial response of a CFM to a 5-s DA bolus is fastest in the outlet center (1.2 $\mathrm{mm}$ diameter), but appreciably slower when the sensor is positioned more laterally as a result of both laminar flow and drag, i.e. both friction between fluid layers and friction between fluid and the tubing wall. The overall width of the CF-MEAs creates limitations regarding placement within the outlet. While the width of the 8-site CF-MEA (CF-MEA-8; $1.12 \mathrm{~mm}$; Fig. 3, middle right) can be positioned within the $1.2 \mathrm{~mm}$ diameter of the tubing outlet, the width of the 16-site CF-MEA (CF-MEA-16; 1.5 mm; Fig. 3, bottom right) exceeds FIA tubing diameter and leads to concern over potential damage of lateral recording sites. Because the CF-MEA-8 can safely be positioned at the flow injection interface, this sensor type was used to determine a height above the outlet that provided a similar temporal response as the interface (Fig. 4A-H). Fig. 4A demonstrates the positioning of the CF-MEA-8 above the tubing outlet in the flow injection apparatus, with channels 4 and 5 closest to the center of the tubing outlet. The CF-MEA-8 was moved dorsally in $0.5-\mathrm{mm}$ increments from $0.0-\mathrm{mm}($ Fig. 4B) to $+2.5-\mathrm{mm}$ above the tubing outlet (Fig. 4G) to determine the effect of distance from the tubing outlet on temporal response to the DA bolus. While all distances tested reached $85 \%$ maximal current within $500 \mathrm{~ms}$, a 1-mm height above the interface demonstrated the fastest temporal response (300 ms to $85 \%$ maximal current, Fig. 4H) and was used for all subsequent sensor response times in this study. Temporal responses 
were normalized to maximal current to characterize response time. Absolute current was used for all other analyses.

The effects of laminar flow and drag based on lateral sensor position are demonstrated in the CFM and CF-MEA-16 in Fig. 5. Sensor placement is depicted in Fig. 5A (top), and the leading edge of the bolus is equivalently recorded when the CFM and central site of a CF-MEA-16 are positioned at similar locations (Fig. 5A, middle and INSET). Background-subtracted voltammograms recorded by FSCV for both sensors are shown underneath the temporal responses, either serially in the pseudocolor plot or individually as a cyclic voltammogram (Fig. 5A, bottom). With an oxidative peak $\approx+0.6 \mathrm{~V}$ and a reductive peak $\approx-0.2 \mathrm{~V}$, voltammograms are characteristic for DA. Figure 5B compares sequential responses recorded when the center site of a CF-MEA-16 was positioned at $100-\mu \mathrm{m}$ increments across the tubing outlet (left) versus responses recorded equivalently but simultaneously at each site of the CF-MEA-16, which are also spaced 100- $\mu \mathrm{m}$ apart (right). In both sets of measurements, effects of laminar flow and drag on the DA bolus is evident at $100-\mu \mathrm{m}$ spatial resolution. A CF-MEA-8 exhibited comparable responses to laminar flow and drag of the DA bolus (Fig. 4I, J, and K, top) and also recorded voltammograms characteristic for DA (Fig. 4K, bottom).

Recording of similar dynamics of the DA bolus by the same CF-MEA site incrementally positioned across the outlet and all CF-MEA sites simultaneously demonstrates that individual site response is more related to position in the measurement field than array. This finding is critical for attributing CF-MEA brain recordings of DA signals with different temporal responses to neural origins (vide infra). 


\section{Performance Metrics}

Several analytical characteristics of DA measurements were assessed with FIA to compare the performance of the CFM, CF-MEA-8, and CF-MEA-16 and are depicted in Figures 6-14. A 5-s bolus of $1-\mu \mathrm{M}$ DA was recorded $1 \mathrm{~mm}$ above the tubing outlet (see Figs. 4A and $\mathbf{5 A}$ ) and analyzed for the following characteristics: response time (Figs. 6-8), sensitivity (Figs. 9 and 10), peak oxidative potential of DA (Fig. 11) RMS noise (Fig. 12), TBC (Fig. 13), and SNR (Fig. 14). The ability to resolve interfering analytes with PCR using the CFM, CF-MEA-8, and CF-MEA16 is demonstrated in Figure 15.

\section{Temporal Response}

Two testing strategies were used to assess temporal response, the elapsed time to $85 \%$ normalized maximal current, in Figures 6-8. For the first strategy, each site of the CF-MEA and each CFM were centered above the outlet. Measurements collected with the first procedure are called individually centered responses times. While time consuming, which limited the analysis to one CF-MEA-16 and CF-MEA-8, these measurements are the most accurate for temporal response because of the effects of laminar flow and drag (Figs. 6 and 7). For the second strategy, each site of CF-MEA was not centered above the outlet. Rather, the position of the CF-MEA-16 was adjusted mediolaterally twice, with one position used to assess sites 1 to 8 (i.e., sites 4 and 5 centered above the outlet), and a second position used to assess sites 9 to 16 (i.e., sites 12 and 13 centered above the outlet). One position was used to assess all CF-MEA-8 sites simultaneously (i.e., sites 4 and 5 centered above the outlet). Measurements collected with the second strategy are called group-centered responses times. This strategy is more time efficient and permitted the analysis of several arrays (4 CF-MEA-16s and 3 CF-MEA-8s; Fig. 8). One important caveat with the second strategy is that array sites were not positioned identically. 
Figure 6 shows individually centered temporal responses normalized to maximal current (top) and in absolute current (bottom) for CFMs (A), individual sites of a CF-MEA-8 (B), and individual sites of a CF-MEA-16 (C). The small contribution of site differences in temporal response due solely to the DA measurement is depicted in Figure 6C (top), which compares the fastest response of each CF-MEA-16 site. All responses reach 85\% maximum within $400 \mathrm{~ms}$ (INSET). Differences in site sensitivity to DA, as shown by recordings of absolute current for the CF-MEA-16 (Fig. 6C, bottom), are rectified by calibration.

Figure 7 shows the statistical analysis of normalized individually centered response times. There was a significant effect of site on response time for the CF-MEA-16 $\left(\mathrm{F}_{(14,30)}=4.41, \mathrm{p}=\right.$ 0.003) and CF-MEA-8 $\left(\mathrm{F}_{(5,12)}=4.16, \mathrm{p}=0.02\right)$, and of sensor on response time for CFMs $\left(\mathrm{F}_{(3,8)}=\right.$ $4.61, \mathrm{p}=0.037)$. However, post-hoc analysis indicated robust consistency of response time across array site and CFM. This analysis is illustrated by the panels showing Tukey-Kramer groupings for the CF-MEA-16 (A), CF-MEA-8 (B), and CFM (C). Solid black boxes within a vertical grouping indicate that values are not statistically significantly different from each other for each type of sensor. Three large clusters of statistically similar response times (13 out of 15 sites for two clusters and 10 out of 15 sites for the third cluster) were found for the CF-MEA-16, and all sites of the CF-MEA-8 exhibited statistically similar response times. With two clusters of similar response times, each with three out of four sensors, CFMs were more variable than the CF-MEA8 but more similar to the CFM-MEA-16. Average response time for the CF-MEA-16, CF-MEA8, and CFM is plotted in D. Statistical analysis failed to find significant $\left(F_{2,22}=3.422, p=0.51\right)$ effect of sensor type on response time. Thus, analysis of individually centered temporal responses indicates excellent consistency of response time between individual sites of each array type and between arrays and CFMs. 
Statistical analysis of normalized group-centered response times is shown in Figure 8. There was a significant effect of array on response time for CF-MEA-16s $\left(\mathrm{F}_{(3,120)}=37.52, \mathrm{p}<\right.$ $0.0001)$ and CF-MEA-8s $\left(\mathrm{F}_{(21,48)}=27.71, \mathrm{p}<0.0001\right)$. There was also a significant effect of site on response time in the nested analysis for CF-MEA-16s $\left(\mathrm{F}_{(56,120)}=5.39, \mathrm{p}<0.0001\right)$ and CFMEA-8s $\left(\mathrm{F}_{(21,48)}=18.69, \mathrm{p}<0.0001\right)$. Panels show Tukey-Kramer groupings for the post-hoc analysis of sites for CF-MEA-16s (A) and CF-MEA-8s (B). Black boxes, indicating statistically similar responses times for groupings, can span across arrays of each type. Tukey-Kramer groupings are the same for all four CF-MEA-16s, and a different set of Tukey-Kramer groupings is the same for all three CF-MEA-8s. Large clusters of statistically similar response times were found within individual CF-MEA-16s (e.g., 14 out of 15 sites for CF-MEA-16(1) and CF-MEA16(2); 13 out of 15 sites for CF-MEA-16(3), and 11 out of 15 sites for CF-MEA-16(4)), and across these arrays (e.g., 40 out of 45 sites across CF-MEA-16(1), (2), and (3); 43 out 60 sites across all sensors). Taken together, these results indicate excellent consistency of response times for the CFMEA-16.

Clusters of statistically similar response times were observed for CF-MEA-8s (e.g., 10 out 24 sites across all arrays), but they appeared smaller compared to those of CF-MEA-16s. The decrease in cluster size for the CF-MEA- 8 when sites are group centered could be due to the larger intra-site spacing (160 $\mu \mathrm{m}$ compared with $100 \mu \mathrm{m}$ for the CF-MEA-16) and the effects of this spacing on temporal response due to laminar flow and drag. Average response times for the CFMEA-16, CF-MEA-8, and CFM are plotted in C top. There was a significant effect of sensor on response time $\left(\mathrm{F}_{7,80}=4.79, \mathrm{p}<0.001\right)$. However, there were two large clusters in which 7 out of 8 sensors and 6 out of 8 sensors exhibited statistically similar response times according to Tukey- 
Kramer groupings (C. bottom). Thus, response times were not only largely consistent within a sensor type but across sensor type as well.

\section{Sensitivity}

Figure 9 compares calibration curves for individual sites of four CF-MEA-16s (A), individual sites of a representative CF-MEA-8 (B), and four CFMs (C). Curves are the best-fit line to five DA concentrations $(0,0.25,0.5,0.75$, and $1 \mu \mathrm{M}$; average of three replicates $)$, the slope $(\mathrm{nA} / \mu \mathrm{M})$ of which is used to determine the sensitivity at each sensing site. Background-subtracted cyclic voltammograms are overlaid for each array site or CFM in top left INSET. Current surrounding the peak oxidative potential for DA is expanded in bottom right INSET. Variability in slope appeared less for the CF-MEA-16s compared to the CF-MEA-8 and CFMs.

The statistical analysis of sensor sensitivity is depicted in Figure 10. There was a significant effect of array on sensitivity for CF-MEA-16s $\left(\mathrm{F}_{(3,120)}=479, \mathrm{p}<0.0001\right)$ and CF-MEA$8 \mathrm{~s}\left(\mathrm{~F}_{(2,48)}=935, \mathrm{p}<0.0001\right)$, and of sensor on sensitivity for CFMs $\left(\mathrm{F}_{(3,8)}=9.02, \mathrm{p}=0.006\right)$. There was also a significant effect of site on sensitivity in the nested analysis for CF-MEA-16s $\left(\mathrm{F}_{(56,120)}\right.$ $=19.53, \mathrm{p}<0.0001)$ and CF-MEA-8s $\left(\mathrm{F}_{(21,48)}=54.44, \mathrm{p}<0.0001\right)$. Panels show Tukey-Kramer groupings for the post-hoc analysis of sites for CF-MEA-16s (A) and CF-MEA-8s (B), and of sensors for CFMs (C). There were clusters of statistically similar sensitivity within individual CFMEA-16s (e.g., 11 out of 15 sites for CF-MEA-16(4); 10 out of 15 sites for CF-MEA-16(3); 9 out of 15 sites for CF-MEA-16(2)), and across arrays (e.g., 21 out of 30 sites across CF-MEA-16(3) and (4); 31 out of 45 sites across CF-MEA-16(2), (3), and (4)).

Clusters of statistically similar sensitivity were also observed for the CF-MEA-8s (e.g., 8 out 24 sites across all arrays), but they appeared less robust compared to those of the CF-MEA16s. With two clusters of similar sensitivity, 2 out of 4 and 3 out of 4 sites, the consistency of 
CFMs appeared similar to that of CF-MEA-16s and greater than that of CF-MEA-8s. Overall, consistency of sensitivity for arrays and CFMs did not appear as robust as for temporal response (see Figs. 7 and 8). Average sensitivity for the CF-MEA-16, CF-MEA-8, and CFM is plotted in D. top. There was a significant effect of sensor on sensitivity $\left(\mathrm{F}_{7,80}=34.93\right.$, $\left.\mathrm{p}<0.001\right)$. CFMs exhibited a significantly higher sensitivity compared to arrays (on average, 27 and 34\% compared to CF-MEA-16s and CF-MEA-8s, respectively; mean sensitivity for CFM-MEA-16s, CF-MEA8s, and CFMs was 8.0, 7.3, and $11 \mathrm{nA} / \mu \mathrm{M}$, respectively). The single solid black box for the first grouping indicates that CFMs are significantly different from all arrays (D. bottom). Across arrays, there was a cluster of 4 out 7 sites with statistically similar sensitivity according to Tukey-Kramer groupings, supportive of consistency of sensitivity across arrays.

\section{Peak Oxidative Potential of Dopamine}

Figure 11 shows the statistical analysis of peak oxidative potential for DA (see Fig. 9 for background-subtracted cyclic voltammogram overlays). Three replicate measurements were taken at each sensing site and analyzed using one-way nested ANOVA (CF-MEA-8s and CF-MEA-16s) and one-way ANOVA (CFMs and between sensor type). There was a significant effect of array on peak oxidative potential for CF-MEA-16s $\left(\mathrm{F}_{(3,120)}=830\right.$, $\left.\mathrm{p}<0.0001\right)$ and CF-MEA-8s $\left(\mathrm{F}_{(2,48)}=\right.$ $1315, \mathrm{p}<0.0001)$, and of sensor on peak oxidative potential for CFMs $\left(\mathrm{F}_{(3,8)}=38, \mathrm{p}<0.0001\right)$. There was also a significant effect of site on peak oxidative potential in the nested analysis for CFMEA-16s $\left(\mathrm{F}_{(56,120)}=2.06, \mathrm{p}=0.0005\right)$ and CF-MEA-8s $\left(\mathrm{F}_{(21,48)}=9.21, \mathrm{p}<0.0001\right)$. Panels show Tukey-Kramer groupings for the post-hoc analysis of sites for CF-MEA-16s (A) and CF-MEA-8s (B) and of sensor for CFMs (C). There were large clusters of statistically similar peak oxidative potentials within individual CF-MEA-16s (e.g., 15 out of 15 sites for CF-MEA-16(1), (2), (3), and (4)) but only one large cluster across CF-MEA-16s (e.g., 29 out of 30 sites across CF-MEA-16(1) 
and (2)). Similarly, there were large clusters of statistically similar peak oxidative potentials within individual CF-MEA-8s (e.g., 8 out of 8 sites for CF-MEA-8(1); 6 out 8 sites for CF-MEA-8(2) and (3)) but more limited clustering across CF-MEA-8s (e.g., 7 out of 16 sites across CF-MEA$8(1)$ and (3)). There was also a large cluster of statistically similar peak oxidative potentials across CFMs (e.g., 3 out of 4 sensors). Peak oxidative potential thus appeared to be highly consistent within an individual array regardless of array type and between CFMs but less consistent across arrays of the same type. Average peak oxidative potential for the CF-MEA-16, CF-MEA-8, and CFM is plotted in D. There was a significant effect of sensor on peak oxidative potential $\left(\mathrm{F}_{7,80}=\right.$ $154, p=0.0001$ ), and there were only two small clusters of statistically similar values for 3 out 8 sensors, including 3 arrays as well as 2 arrays and the CFMs (Fig. 11D, bottom). Thus, peak oxidative potential of CFMs was similar to that for some arrays. The robust consistency of this aspect of electrochemistry across sites within an array will be important for streamlining the timeconsuming technique of PCR for resolving DA from multianalyte FSCV recordings. It should be noted that data were collected over multiple days, and it is possible that electrochemical variation between sensors is due to testing variation (i.e. reference electrode, Tris buffer) rather than difference in sensor performance.

\section{Root-Mean-Square Noise}

The results of RMS noise analysis are shown in Figure 12. RMS noise was calculated using ten consecutive current tracings collected at the peak oxidative potential for DA. There was a significant effect of array on RMS noise for CF-MEA-16s $\left(\mathrm{F}_{(3,120)}=20.75, \mathrm{p}<0.0001\right)$ and CFMEA-8s $\left(\mathrm{F}_{(2,48)}=12.5, \mathrm{p}<0.0001\right)$ but not of sensor on RMS noise for CFMs $\left(\mathrm{F}_{(3,8)}=0.96, \mathrm{p}=\right.$ 0.50). There was also a significant effect of site on RMS noise in the nested analysis for CF-MEA$16 \mathrm{~s}\left(\mathrm{~F}_{(56,120)}=28.94, \mathrm{p}<0.0001\right)$ and CF-MEA-8s $\left(\mathrm{F}_{(21,48)}=5.67, \mathrm{p}<0.0001\right)$. Panels show Tukey- 
Kramer groupings for the post-hoc analysis of sites for CF-MEA-16s (A) and CF-MEA-8s (B). No groupings are shown for CFMs $(\mathbf{C})$ because no significant main effect was found. There were large clusters of statistically similar RMS noise within individual CF-MEA-16s (e.g., 15 out of 15 sites for CF-MEA-16(1) and (2); 14 out of 15 sites for CF-MEA-16(4); 12 out of 15 sites for CFMEA-16(3)), and across CF-MEA-16s (e.g., 30 out of 30 sites across CF-MEA-16(1) and (2); 56 out of 60 sites across all arrays). There were also large clusters of statistically similar RMS noise within individual CF-MEA-8s (e.g., 8 out of 8 sites for CF-MEA-8(2); 6 out of 8 sites for CFMEA-8(1) and (2)), and across CF-MEA-8s (e.g., 20 out of 24 sites across all arrays). These results, in combination with no main effect of sensor for RMS noise of CFMs, indicates robust consistency of RMS noise within sensor type. Average RMS noise for the CF-MEA-16, CF-MEA8, and CFM is plotted in D. There was no significant effect of sensor on RMS noise $\left(F_{7,80}=0.672\right.$, $p=0.695)$, supportive of the consistency of this analytical characteristic across sensor type.

\section{Total Background Current}

Figure 13 shows the statistical analysis of TBC. TBC was determined by summing the absolute value of current readings at all 1,000 points in the background cyclic voltammogram. There was a significant effect of array on TBC for CF-MEA-16s $\left(\mathrm{F}_{(3,120)}=14603, \mathrm{p}<0.0001\right)$ and CF-MEA-8s $\left(\mathrm{F}_{(2,48)}=4414, \mathrm{p}<0.0001\right)$, and of sensor on TBC for CFMs $\left(\mathrm{F}_{(3,8)}=3318, \mathrm{p}<\right.$ 0.0001). There was also a significant effect of site on TBC in the nested analysis for CF-MEA-16s $\left(\mathrm{F}_{(56,120)}=10672, \mathrm{p}<0.0001\right)$ and CF-MEA-8s $\left(\mathrm{F}_{(21,48)}=798, \mathrm{p}<0.0001\right)$. Panels show TukeyKramer groupings for the post-hoc analysis of sites for CF-MEA-16s (A) and CF-MEA-8s (B), and of sensors for CFMs (C). Clusters of statistically similar TBCs were small and sparse within and among arrays and between CFMs. While TBC appeared to be the least consistent analytical characteristic within sensor type, it should be considered that this result may be due to the low 
variability of the stable TBC, which increases statistical power for identifying differences. Average TBC for the CF-MEA-16, CF-MEA-8, and CFM is plotted in D. While there was a significant effect of sensor on $\mathrm{TBC}\left(\mathrm{F}_{7,80}=2.30, \mathrm{p}=0.034\right)$, post-hoc analysis failed to identify a significant pairwise difference. Thus, despite poor consistency of TBC within sensor type, no sensor was found to be significantly different from another.

\section{Signal-to-Noise Ratio}

The statistical analysis of SNR is depicted in Figure 14. SNR was calculated by dividing maximal current of the signal produced by $1 \mu \mathrm{M}$ DA bolus by RMS noise. There was a significant effect of array on SNR for CF-MEA-8s $\left(\mathrm{F}_{(2,48)}=10.55, \mathrm{p}<0.0002\right)$ but not for CF-MEA-16s $\left(\mathrm{F}_{(3,120)}=2.03, \mathrm{p}=0.114\right)$ or of sensor on SNR for CFMs $\left(\mathrm{F}_{(3,8)}=1.36, \mathrm{p}=0.32\right)$. There was also a significant effect of site on SNR in the nested analysis for CF-MEA-16s $\left(\mathrm{F}_{(56,120)}=2.18, \mathrm{p}<\right.$ 0.0001) and CF-MEA-8s $\left(\mathrm{F}_{(21,48)}=2.72, \mathrm{p}<0.0001\right)$. Panels show Tukey-Kramer groupings for the post-hoc analysis of sites for CF-MEA-16s (A) and CF-MEA-8s (B). No groupings are shown for CFMs (C) because no significant main effect was found. There were large clusters of statistically similar SNR within individual CF-MEA-16s (e.g., 15 out of 15 sites for CF-MEA16(1) and (2); 14 out of 15 sites for CF-MEA-16(3) and (43)), and across CF-MEA-16s (e.g., 30 out of 30 sites across CF-MEA-16(1) and (2); 44 out of 45 sites across CF-MEA(1), (2), and (4); 56 out of 60 sites across all arrays). There were also large clusters of statistically similar RMS noise within individual CF-MEA-8s (e.g., 8 out of 8 sites for CF-MEA-8(1), (2), and (3)), and across CF-MEA-8s (e.g., 22 out of 24 sites across all arrays). SNR thus appeared to be the most consistent analytical characteristic within sensor type. Average SNR for the CF-MEA-16, CFMEA-8, and CFM is plotted in D. In agreement with consistency within sensor type, statistical analysis failed to find a significant effect of sensor on $\operatorname{SNR}\left(\mathrm{F}_{7,80}=1.75, \mathrm{p}=0.11\right)$. Interestingly, 
SNR was therefore the most consistent analytical characteristic overall; however, this runs counter to our finding that sensitivity varied significantly between sensor type. This finding is important for in vivo measurements because SNR determines detection limit.

\section{Principle Component Regression}

The ability of CF-MEAs to resolve a mixed analyte signal recorded by FSCV into individual analytes using the approach of PCR is demonstrated in Figure 15. PCR is a combined approach utilizing principle component analysis and inverse least-squares regression that has been applied to resolve DA from a multianalyte raw FSCV record obtained by a CFM (Heien et al., 2004; Keithley et al., 2009). For this test, PCR was used to resolve individual responses for DA (Fig. 15A) and a change in $\mathrm{pH}(\Delta \mathrm{pH}$; Fig. 15B $)$ from mixtures of DA and $\Delta \mathrm{pH}$ (Fig. 15C-D). The pH of brain extracellular fluid is dynamic and changes with neuronal activity (Venton et al., 2003). While $\mathrm{H}^{+}$is not electroactive, the oxidation and reduction of chemical groups on the surface of the carbon fiber are $\mathrm{pH}$ sensitive (Kawagoe et al., 1993). Thus, $\Delta \mathrm{pH}$ will generate a temporal response and characteristic voltammogram (Fig. 15B). More importantly, $\Delta \mathrm{pH}$ distorts the DA current measured at the peak oxidative current for DA in the temporal response when both analytes are present (Fig. 15C-D). The two mixtures of DA $(1-\mu \mathrm{M})$ and $\Delta \mathrm{pH}$ were selected because the acidic $\mathrm{pH}$ (7.15) adds to the DA temporal response while the basic $\mathrm{pH}$ (7.65) subtracts from the DA temporal response (Fig. 15C-D). The $\mathrm{pH}$ of the calibration buffer flowing across the sensor prior to analyte injection in FIA is 7.4. As a result, $\Delta \mathrm{pH}$ responses represent the difference from a baseline $\mathrm{pH}$ of 7.4 .

As shown in Figure 15C and D, a single representative site on a CF-MEA-16 or CF-MEA8 was able to revolve DA and $\Delta \mathrm{pH}$ from a mixture of the two, similar to the performance of a CFM

for PCR. Agreement between observed (measured) and predicted (PCR-resolved) responses for 
the two analytes was statistically significant for all sensors as assessed by the Pearson correlation coefficient. This result underscores the ability of CF-MEAs to operate successfully for FSCV in the chemically complex extracellular milieu of the brain. 


\section{CHAPTER IV: RESULTS - IN VIVO CHARACTERIZATION OF CF-MEAs}

\section{Background}

The measurement of DA signaling in the midbrain provides insight into the neurological basis of learning and behavior. The striatum is a midbrain region heavily innervated by DA neurons, and these DA neurons signal in distinct tonic and phasic modes in vivo to signal target neurons (Schultz, 2007; Dreyer et al., 2010). In the tonic mode, DA neurons discharge irregularly at low frequencies $(\approx 5 \mathrm{~Hz})$ (Grace and Bunney, 1984) and establish a baseline level of extracellular DA. This DA tone enables movement, cognition, and motivation (Schultz, 2007). In response to certain stimuli, including novelty, salient stimuli, social interaction, and reward, DA neurons fire in bursts of action potentials at higher frequencies $(>15 \mathrm{~Hz})$ to signal and reinforce the value of performing advantageous behaviors (Floresco et al., 2003; Robinson et al., 2011; Hamid et al., 2016; Mohebi et al., 2019). During this phasic mode of signaling, action potential bursts lead to sub-second to second increases in extracellular DA called transients that ride on top of DA tone (Garris and Keefe, 2015) and are detectable by conventional FSCV. DA transients drive the learning of predictive cues and play a significant role in reward learning (Covey et al., 2014) suggesting alteration of their frequency, amplitude, and duration will lead to modification of behavior. Indeed, drugs of abuse, particularly amphetamine (AMPH), are known to increase the frequency, amplitude, and duration of DA transients.

\section{CF-MEAs Capture Heterogeneity of Electrically Evoked Dopamine Signals}

The urethane-anesthetized preparation was used to assess the performance of the CF-MEA16 in vivo and demonstrates the heterogeneity of electrically evoked DA signals in the striatum

(Fig. 16A). The heterogeneous nature of the striatum results, at least in part, from a patchwork of distinct DA kinetic domains that are characterized by a variety of DA signal types. Fast and slow 
kinetic domains in the striatum are distinguishable by examining the amplitude and temporal profile of electrically evoked DA signals (Moquin and Michael 2009). Striatal DA signals can be categorized into fast and slow domains, with fast DA signals further subdivided into four different types based on release kinetics (Types 1-4; Taylor et al. 2015). In fast domains, DAT favors both rapid DA release and uptake. In the slow domain, DAT favors non-action potential-dependent DA efflux and slow DA uptake. The resulting high levels of DA tone caused by these factors inhibit DA release by acting on autoreceptors. Conventionally, CFMs have been optimized in vivo for a single fast DA signal type (Type 1), and comparatively little work has been done to characterize the remaining types more fully. Understanding of these previously unexplored signal types may provide further insight into the underlying mechanisms of reward learning. Moreover, the striatum is also anatomically divided into patch and matrix compartments, each with distinct afferent (input) and efferent (output) connections and more importantly, distinct DA dynamics (Brimblecombe \& Cragg, 2015, 2017; Salinas et al., 2016). DA neurons innervate both patch and matrix compartments but are denser in patch. Additionally, DA release differs between patch and matrix compartments in distinct anatomic locations. In the dorsal striatum, DA release is greater in the matrix, whereas DA release is greater in the patch in the ventral striatum (Brimblecombe \& Cragg, 2015).

A CF-MEA-16 was implanted in the dorsomedial striatum and a stimulating electrode was implanted in the medial forebrain bundle to electrically activate ascending DA axons. Previous work using FSCV at a CFM in anesthetized rats has demonstrated a profound heterogeneity of electrically evoked DA signal amplitude and dynamics in the striatum (May and Wightman, 1989; Garris et al., 1994) that have more recently been classified by type (Walters et al., 2015; Taylor et al., 2015). With a similar recording site spacing $(100 \mu \mathrm{m})$ as the striatal 
heterogeneity previously described by the CFM, the CF-MEA-16 is potentially well suited for capturing the different dynamics and amplitudes of these DA signals with similar high fidelity but simultaneously across a much larger measurement field. Thus, monitoring the heterogeneity of evoked striatal DA signals is a robust test of the CF-MEA-16 that will also provide additional insight into the heterogeneity of DA neurotransmission in the striatum.

Figure 16A describes measurements of electrically evoked transient-like DA signals as the CF-MEA-16 is lowered in $100 \mu \mathrm{m}$-increments ventrally in the striatum. The position of the stimulating electrode, which applied 0.4-s (24 pulse) trains, was initially optimized using a CFM and fixed in the medial forebrain bundle for all measurements. The heat map (top) visibly shows heterogeneity of the maximal concentration of DA evoked by the electrical stimulation ([DA $]_{\max }$ ) within the recorded portion of the striatum. Each row represents a simultaneous recording at each site (100- $\mu$ m resolution) of the CF-MEA-16, while columns represent recordings taken separately as the CF-MEA-16 is lowered in 100- $\mu \mathrm{m}$ increments. Recordings from a CFM lowered earlier in the experiment through approximately the middle of this recorded region demonstrate similar heterogeneity. Dynamic information about the DA signals is revealed by individual temporal responses at select sites (Fig. 16, bottom). Consistent with similar response times demonstrated by FIA (see Fig 6), in vivo signals of similar amplitude and recorded by an individual site on the CFMEA-16 and a CFM substantively overlay.

Differences in the temporal responses electrically evoked by a longer stimulus train ( 3 versus $0.4 \mathrm{~s}$ ) form the basis for categorizing striatal DA signals into fast and slow, with fast signals further subdivided into types 1 to 4 (Taylor et al., 2015; Fig. 17). Fast and slow domains in the striatum exhibit distinct regulation of extracellular DA levels and pharmacological profiles (Moquin and Michael, 2009; Moquin and Michael, 2011; Taylor et al., 2012). The CF-MEA-16 
uniquely identified fast and slow types of electrically evoked DA signals and all but one (type 3) of the fast types (Fig. 17A) across a broad region of the dorsal striatum. Figure 17B illustrates the temporal and dynamic profiles of the kinetically diverse DA signals observed, with Types 1,2 , and 4 fast signals leading to increased concentrations of DA detected within $200 \mathrm{~ms}$. The slow domain identified takes appreciably longer to rise above the detection limit.

This striatal heterogeneity also extends more laterally as demonstrated by a different CFMEA-16 implanted in another rat (Fig. 18A), indicating that even the expanded number of recording sites for FSCV offered by the CF-MEA-16 compared to a CFM does not fully capture the richness of striatal DA signals. All but one recording location in the striatum in which 0.4-s trains failed to detect DA (Fig. 18A) produced measurable DA signals when evoked by 3-s trains (Fig 18B). Surprisingly, most recording sites with undetectable DA signals were type 1, which exhibited the highest $[\mathrm{DA}]_{\max }$ evoked by 0.4-s trains (Fig. 18C). Additional work is needed to investigate these interesting and surprising findings.

\section{In Vivo CF-MEA Characteristics}

Of critical importance is determining both the stability of sensors over time as well as establishment of dynamic sensing range. Signals recorded at the same site over a period of $25 \mathrm{~min}$ are stable (Fig. 19A). Moreover, the punctate nature of the stability heat map suggests that gradual changes in DA sensitivity over time are not responsible for the differences in $[\mathrm{DA}]_{\max }$ observed during depth studies (see Figs. 16A and 18A). The ability of the CF-MEA-16 to record signals of different amplitudes faithfully in vivo is supported by site-specific increases in DA concentration with increasing stimulus pulse number (Fig. 19B).

Prior FIA characterization of CF-MEA-16s demonstrated similar SNR for DA monitoring with FSCV as CFMs. This result is critical for establishing the potential of CF-MEAs for in vivo 
monitoring of all DA signals previously characterized by CFMs, particularly signals with low amplitudes near the detection limit. To assess whether this similarity of SNR holds true after implantation in the brain, in vivo SNRs for the CF-MEA-16 and CFM were compared. SNRs in Figure 20A and B top were calculated from DA signals electrically evoked by 0.4-s trains and shown in Figure 16A and Figure 18A for two different pairings of a CF-MEA-16 and CFM in different animals, respectively. Measurements with the single CFM or three CFMs were collected first. After CFM removal, the array was implanted to a similar mediolateral, anteroposterior, and dorsoventral location in the striatum. Not unexpectedly, there is heterogeneity of SNR that appears to mirror $[\mathrm{DA}]_{\max }$ for both CF-MEA-16 and CFM. For a given pair of sensors, the heterogeneity of SNR also appears similar for the CF-MEA-16 and CFM, at least for some sub-regions of the striatum.

To quantitatively compare SNR between the two sensor types, values for the three most middle sites of each array were averaged. These three sites approximate the mediolateral location of the single CFM in Figure 20A and the middle of the three CFMs in Figure 20B. The other two CFMs in Figure 20B are located either more medial or lateral to the array. Although equivalent recording locations are approximate for each array and associated CFM, the location of the stimulating electrode is fixed for all measurements in each animal, which promotes comparison SNRs. Figures 20A and B bottom show average SNR for each pairing of CF-MEA-16 and CFM. SNR was not different between the array and CFM in Figure 20A $\left(\mathrm{t}_{22}=1.378 ; \mathrm{p}=0.18\right)$ or Figure 20B $\left(\mathrm{t}_{18}=0.7413 ; \mathrm{p}=0.47\right)$. These in vivo results for SNR are consistent with those from FIA and thus highlight the similarity of this important analytical characteristic between the CF-MEA-16 and CFM for monitoring striatal DA signals in vivo. 


\section{CF-MEAs Capture Effects of AMPH on Baseline and Phasic Dopamine Signals}

The psychostimulant amphetamine (AMPH) is a potent inhibitor of DAT that exhibits therapeutic efficacy in the treatment of attention deficit disorder (ADD)/ attention deficit hyperactivity disorder (ADHD) and narcolepsy (Sulzer et al., 2005; Arnsten, 2006). Because DAT inhibition increases extracellular levels of brain DA, particularly in those regions involved in reward learning (DiChiara and Imperato, 1988), AMPH is also known to exhibit significant abuse potential (Rothman and Baumann, 2006; Berman et al. 2008;). While the mechanism of action by which AMPH increases brain DA levels has been extensively studied for decades (Sulzer et al., 2005), more recent work has aimed at providing insight into the presynaptic mechanisms of DA release and uptake (Covey et al., 2014). The complexity of DA signaling is increased in the presence of AMPH due to its ability to both block and reverse the function of DAT as well as its ability to augment vesicular DA release (Avelar et al., 2013). At this point, AMPH-induced changes in DA concentration are incompletely understood and hypothesized to be a result of action-potential independent DA efflux, action-potential dependent exocytotic release events, or a combination of the two.

As expected, AMPH modulates electrically evoked DA signals in the striatum. The effects of AMPH (1.0-mg/kg i.v.) on electrically evoked transient-like DA signals in the striatum of the anesthetized rat and assessed by a CF-MEA-16 are shown in Figure 21. While there is heterogeneity in the pre-AMPH DA signals (Fig. 21A, left) as previously shown above for these 0.4-s stimulus trains (see Figs. 16A and 18A), AMPH increased the amplitude of temporal responses at all sites (Fig. 21A, right). The drug-induced increase in signal amplitude is voltammetrically attributed to DA. A heat map highlights the spatially heterogenous nature of these AMPH effects, both in terms of absolute and relative increases in $[D A]_{\max }($ Fig. 21B, first 
panel). Similar to effects on amplitude as demonstrated by the raw recordings, AMPH increased $[\mathrm{DA}]_{\max }$ at all sites.

Extracellular DA levels are tightly controlled by the presynaptic mechanisms of DA release and uptake, which can be resolved using mathematical means from electrically evoked DA signals measured by FSCV at a CFM (Wightman et al., 1988;Wu et al., 2001). After signal conditioning to remove the effects of DA adsorption to the surface of the carbon fiber (i.e., "hang-up"), temporal responses recorded by the CF-MEA-16 were kinetically analyzed for three parameters, $R_{\mathrm{p}}, \mathrm{k}_{\mathrm{U}}$, and $\mathrm{k}_{\mathrm{T}}$, describing DA release, uptake, and diffusion, respectively (Walters et al., 2014; Walters et al., 2015). Similar to [DA $]_{\max }$ (Fig. 21B, first panel), AMPH increased the release term, $\mathrm{R}_{\mathrm{p}}$, at all sites (Fig. 21B, second panel). In contrast, AMPH decreased the uptake term, $\mathrm{k}_{\mathrm{U}}$, at all sites (Fig. 21B, third panel), consistent with its established mechanism of a competitive DA uptake inhibitor (Sulzer et al., 2005). Previous in vivo studies using FSCV at a CFM to record electrically evoked transient-like DA signals have also reported AMPH-induced increases in DA release and decreases in DA uptake (Daberkow et al., 2013;Covey et al., 2013). The decrease in the diffusion term $\mathrm{k}_{\mathrm{T}}$, with AMPH (Fig. 21B, fourth panel) is a novel finding, but it has been described previously for another competitive DA uptake inhibitor, GBR12909, using an identical kinetic model (Walters et al., 2020). However, analysis with an expanded model attributed this decrease in $\mathrm{k}_{\mathrm{T}}$ to DA uptake. Nevertheless, it is interesting to note that, like [DA $]_{\max }$, parameters, and the effects of AMPH on these parameters were spatially heterogeneous. This result underscores the spatial coverage afforded by CF-MEAs for pharmacological investigations of DA presynaptic mechanisms in vivo but with the exquisite analytical characteristics of the CFM.

Using FSCV at a CFM, AMPH has been show to increase DA baseline via an unknown mechanism as well as the frequency, amplitude, and duration of DA transients (Daberkow et al., 
2013; Covey et al., 2013; Covey et al., 2016). In contrast to electrically evoked measurements using FSCV, whose chemical identify is established by voltammograms (vide supra), phasic DA signals and changes in DA baseline require more robust means to determine analyte identify. In particular, changes in extracellular $\mathrm{pH}$ reflecting neuronal activity and sensor drift distort the voltammetric measurement of DA over longer periods of time and must be isolated using PCR. Demonstrating the ability of CF-MEAs to monitor changes in DA baseline as well as phasic DA signals is thus critical for establishing this sensor approach for investigations targeting naturally occurring and drug-evoked DA signals.

Pre-drug (i.e., non-drug) recordings from a CF-MEA-16 implanted in the striatum of an anesthetized rat failed to detect either DA transients or changes in baseline DA levels when resolved by PCR (Fig. 22A). In sharp contrast, i.v. administration of 1.0-mg/kg AMPH robustly activated DA transients (post-AMPH; Fig. 22B and C). DA transients occurred synchronously in rapid succession, to the extent causing a gradual increase in extracellular DA, three minutes after drug administration (Fig. 22B). Interestingly, five minutes after drug administration, individual transients were detectable at some, but not all, sites of the CF-MEA-16 (Fig. 22C).

To assess the ability to monitor longer-duration changes in extracellular DA, AMPH (10 $\mathrm{mg} / \mathrm{kg}$ ) was combined with the $\mathrm{D}_{2} \mathrm{DA}$ antagonist raclopride $(2.0 \mathrm{mg} / \mathrm{kg})$ and administrated i.p. AMPH has been shown to induce inhibition of burst firing in DA cells by increasing extracellular DA levels, particularly by activating $\mathrm{D}_{2} \mathrm{DA}$ receptors, and this effect is compounded in the anesthetized rat preparation. Both effects can be overcome with administration of raclopride (Shi et al., 2000). Indeed, a cocktail of psychostimulant and D2 DA antagonist administered via this route has previously been shown to elicit a variety of DA signals, including some with a duration of several seconds (Robinson et al., 2014). This drug cocktail elicited a large ( $>0.5 \mu \mathrm{M})$ and 
extended (several minutes) increase in DA baseline that was detected at all sites of the CF-MEA16, albeit to varying degrees, (Fig. 22D). In contrast, a shorter duration $(\approx 30$ s $)$ and smaller $(\approx 200$ to $300 \mu \mathrm{M}$ ) increase in DA baseline was only detected at a few recording sites (Fig. 22E). 


\section{CHAPTER V: CONCLUSION}

Thorough FIA characterization suggests these self-insertable CF-MEAs are generally similar to conventional CFMs in terms of response time, RMS noise, TBC, and SNR. One difference across sensors was the peak oxidative potential aspect of electrochemistry. Although peak oxidative potential differed across sensors, it was similar between CFMs and some arrays. Another difference across sensors was sensitivity, which was somewhat greater for CFMs compared to all arrays $(\approx 30 \%$ on average $)$, and this result runs counter to the findings that RMS noise and SNR ratio did not vary across sensors in either in vivo or FIA applications. While we do not have a ready explanation for this discrepancy, it is entirely possible that differences in RMS noise between sensors escaped our statistical analysis. Nevertheless, it should be emphasized that as the determinant of detection limit, the critical analytical characteristic of SNR was similar between arrays and CFMs. It is also important to consider the robust consistency of SNR within and between sites of arrays, indicating that each site and array will bring a similar detection limit to monitoring DA signals.

Collectively considering the analysis of temporal response and SNR, results indicate that CF-MEAs are capable of detecting DA signals of the same dynamics and amplitude as CFMs. Moreover, the robust consistency of the arrays with regard to these analytical characteristics afford the important advantage that each site of an array and each array will be as similarly capable as the CFM for monitoring DA signals. Overall, the analytical characteristics of CF-MEA-8 and CFMEA-16 for DA monitoring with FSCV thus compare favorably with those of the CFM.

In vivo, CF-MEAs identified fast and slow types of electrically evoked DA signals and four out of five previously described signal types across a broad region of the dorsal striatum. Moreover, we have demonstrated heterogeneity in both electrically evoked $[\mathrm{DA}]_{\max }$ as well as the 
kinetic parameters associated with modeling phasic DA signaling events. Finally, we have shown that AMPH modulated electrically evoked transient-like DA signals, changes in baseline DA concentration, and phasic DA transients are robustly detectable by CF-MEAs. Taken together, these results demonstrate that CF-MEAs afford a similar utility as CFMs for monitoring brain DA signals in a temporally, chemically, and spatially resolved manner while providing the transformative advantage of substantively expanded spatial coverage for superior characterization.

Given the favorable performance of CF-MEAs in this study, the use of this class of microsensors in future full-scale studies is seemingly justified. While we observed changes in baseline DA levels in the presence of AMPH, it should be emphasized that the mechanism of AMPH action on increasing DA tone is not established. Future investigations utilizing CF-MEAs may help reconcile discrepant results regarding action potential-dependent and independent actions of AMPH on DA signaling (Sulzer et al., 2005;Covey et al., 2014). Additional work is also needed to characterize the utility of CF-MEAs more fully in the freely behaving animal. 


\section{REFERENCES}

Arnsten AF (2006) Stimulants: Therapeutic actions in ADHD. Neuropsychopharmacology $31: 2376-2383$.

Atcherley CW, Laude ND, Parent KL, Heien ML (2013) Fast-scan controlled-adsorption voltammetry for the quantification of abolute concentrations and adsorption dynamics. Langmuir 29:14885-14892.

Atcherley CW, Wood KM, Parent KL, Hashemi P, Heien ML (2015). The coaction of tonic and phasic dopamine dynamics. Chem Commun 51:2235-2238.

Avelar AJ, Juliano SA, Garris PA (2013) Amphetamine augments vesicular dopamine release in the dorsal and ventral striatum through different mechanisms. J Neurochem 125:373-385.

Bermann S, O’Neill J, Fears S, Bartzokis G, London ED (2008) Abuse of amphetmines and structural abnormalities in brain. Ann N Y Acad Sci 1141:195-220.

Brimblecombe KR, Cragg SJ (2015) Substance P weights striatal dopamine transmission differently within the striosome-matrix axis. J Neurosci 35:9017-9023.

Brimblecombe KR, Cragg SJ (2017) The striosome and matrix compartments of the striatum: a path through the labyrinth from neurochemistry toward function. ACS Chem Neurosci $8: 235-242$.

Chang SY, Kim I, Marsh MP, Jang DP, Hwang SC, Van Gompel JJ, Goerss SJ, Kimble CJ, Bennet KE, Garris PA, Blaha CD, Lee KH (2012) Wireless fast-scan cyclic voltammetry to monitor adenosine in patients with essential tremor during deep brain stimulation. Mayo Clin Proc 87:760-765. 
Clark JJ, Sandberg SG, Wanat MJ, Gan JO, Horne EA, Hart AS, Akers CA, Paker JG, Willuhn I, Martinez V, Evans SB, Stella N, Phillips PEM (2010) Chronic microsensors for longitudinal, subsecond dopamine detection in behaving animals. Nat Methods 7:126129.

Covey DP, Juliano SA, Garris PA (2013) Amphetamine elicits opposing actions on readily releasable and reserve pools for dopamine. PLoS One 8:e60763.

Covey DP, Roitman MF, Garris PA (2014) Illicit dopamine transients: Reconciling actions of abused drugs. Trends Neurosci 37:200-210.

Covey DP, Bunner KD, Schuweiler DR, Cheer JF, Garris PA (2016) Amphetamine elevates nucleus accumbens dopamine via an action potential-dependent mechanism that is modulated by endocannabinoids. Eur J Neurosci 43:1661-1673.

Daberkow DP, Brown HD, Bunner KD, Kraniotis SA, Doellman MA, Ragozzino ME, Garris PA, Roitman MF (2013) Amphetamine paradoxically augments exocytotic dopamine release and phasic dopamine signals. J Neurosci 33:452-463.

Day JJ, Roitman MF, Wightman RM, Carelli RM (2007) Associative learning mediates dynamic shifts in dopamine signaling in the nucleus accumbens. Nat Neurosci 10:1020-1028.

Da Silva JA, Tecuapetla F, Paixao V, Costa RM (2018) Dopamine neuron activity before action initiation gates and invigorates future movements. Nature 554:244-248.

Di Chiara G, Imperato A (1988) Drugs abused by humans preferentially increase synaptic dopamine concentrations in the mesolimbic system of freely moving rats. P Natl Acad Sci USA 85:5274-5278. 
Dreyer JK, Herrik KF, Berg RW, Hounsgaard JD (2010) Influence of phasic and tonic dopamine release on receptor activation. J Neurosci 30:14273-14283.

Everitt BJ, Robbins TW (2005) Neural system of reinforcement for drug addiction: from action to habits to compulsion. Nat Neurosci 8:1481-1489.

Flagel SB, Clark JJ, Robinson TE, Mayo L, Czuj A, Willuhn I, Akers CA, Clinton SM, Phillips PEM, Akil H (2011) A selective role for dopamine in stimulus-reward learning. Nature 469:53-57.

Floresco SB, West AR, Ash B, Moore H, Grace AA (2003) Afferent modulation of dopamine neuron firing differentially regulates tonic and phasic dopamine transmission. Nat Neurosci 6:968-973.

Fortin SM, Cone JJ, Ng-Evans S, McCutcheon JE, and Roitman MF (2016) Sampling phasic dopamine signaling with fast-scan cyclic voltammetry in awake behaving rats. Curr Prot Neurosci 70:70.25.1-7.25.20

Gale SD, Perkel DJ (2006) Physiological Properties of Zebra Finch Ventral Tegmental Area and Substantia Nigra Pars Compacta Neurons. J neurophysiol 96:2295-2306.

Gan JO, Walton ME, Phillips PEM (2010) Dissociable cost and benefit encoding of future rewards by mesolimbic dopamine. Nat Neurosci 13:25-27.

Garris PA, Ciolkowski EL, Wightman RM (1994) Heterogeneity of evoked dopamine overflow within the striatal and striatoamygdaloid regions. Neuroscience 59:417-427.

Garris PA, Christensen JRC, Rebec GV, Wightman RM (1997) Real-time measurement of electrically evoked extracellular dopamine in the striatum of freely moving rats. J Neurochem 68:152-161 
Garris PA, Keefe KA (2015) Voltammetric analysis of loss and gain of dopamine function. In Compendium of in vivo monitoring real-time molecular neuroscience. Wilson GS, Michael AC, Eds. World Scientific Publishing Co.:Hackensack, NJ.

Glanowska KM, Venton BJ, Moenter SM (2012) Fast Scan Cyclic Voltammetry (FSCV) as a novel method for detection of real time gonadotropin-releasing hormone $(\mathrm{GnRH})$ release in mouse brain slices. J Neurosci 32:14664-14669.

Grace AA and Bunney BS (1984) The control of firing pattern in nigral dopamine neurons: burst firing. J Neurosci 4:2877-2890.

Grace AA, Floresco SB, Goto Y, Lodge DJ (2007) Regulation of firing of dopaminergic neurons and control of goal-directed behaviors. Trends Neurosci 30:220-227.

Greco PG, Meisel RL, Heidenreich BA, Garris PA (2006) Voltammetric measurement of electrically evoked dopamine levels in the striatum of the anesthetized Syrian hamster. J Neuro Meth 152:55-64.

Guo Q, Zhou J, Feng Q, Lin R, Gong H, Luo Q, Zeng S, Luo M, Fu L (2015) Multi-channel fiber photometry for population neuronal activity recording. Biomed Opt Express 6:246854

Hamid AA, Pettibone JR, Mabrouk OS, Hetrick VL, Shmidt R, Vander Weele CM, Kennedy RT, Aragona BJ, and Berke JD (2016) Mesolimbic dopamine signals the value of work. Nat Neursci 19:117-126.

Hashemi P, Dankoski EC, Wood KM, Ambrose RE, Wightman RM (2011) In vivo electrochemical evidence for simultaneous 5-HT and histamine release in the rat substantia nigra pars reticulata following medial forebrain bundle stimulation. J Neurochem 118:749759. 
Heien MLAV, Phillips PEM, Stuber GD, Seipel AT, Wightman RM (2003) Overoxidation of carbon-fiber microelectrodes enhances dopamine adsorption and increases sensitivity. Analyst 128:1413-1419.

Heien MLAV, Johnson MA, Wightman RM (2004) Resolving neurotransmitters detected by fast-scan cyclic voltammetry. Anal Chem 76:5697-5704.

Heien MLAV, Khan AS, Ariansen JL, Cheer JF, Phillips PEM, Wassum KM, and Wightman RM (2005) Real-time measurement of dopamine fluctuations after cocaine in the brain of behaving rats. PNAS 102:10023-10028

Howe MW, Tierney PL, Sandberg SG, Phillips PEM, Graybiel AM (2013) Prolonged dopamine signaling in striatum signals proximity and value of distant rewards. Nature 500:575-579.

Huffman ML, Venton BJ (2009) Carbon-fiber microelectrodes for in vivo applications. Analyst 134:18-24.

Jaquins-Gerstl A, Michael AC (2015) A review of the effects of FSCV and microdialysis measurements on dopamine release in the surrounding tissue. Analyst 140:3696-3708.

Jarriault D, Fuller J, Hyland BI, Mercer AR (2018) Dopamine release in mushroom bodies of the honey bee (Apis mellifera L.) in response to aversive stimulation. Sci Rep 8:16277.

Jing M, Zhang Y, Wang H, and Li Y (2019) G-Protein-coupled receptor-based sensors for imaging neurochemicals with high sensitivity and specificity. J Neurochem 151:279-288.

Kawagoe KT, Garris PA, Wightman RM (1993) pH-Dependent processes at Nafion-coated carbon-fiber microelectrodes. J Electroanal Chem 359:193-197.

Keiflin R, Janak PH (2015) Dopamine Prediction Errors in Reward Learning and Addiction: From Theory to Neural Circuitry. Neuron 88:247-263. 
Keithley RB, Heien ML, Wightman RM (2009) Multivariate concentration determination using principal component regression with residual analysis. Trends Analyt Chem 28:11271136.

Kim MH, Yoon H, Choi SH, Zhao F, Kim J, Song KD, Lee U (2016) Miniaturized and wireless optical neurotransmitter sensor for real-time monitoring of dopamine in the brain. Sensors 16:1894.

Lammel S, Lim BK, Malenka RC (2014) Reward and aversion in a heterogeneous midbrain dopamine system. Neuropharmacology 76:121-128.

Li N, Jasanoff A (2020) Local and global consequences of reward-evoked striatal dopamine release. Nature 580:239-244.

Lin MZ, Schnitzer MJ (2016) Genetically encoded indicators of neuronal activity. Nat Neurosci 19:1142-1153.

May LJ, Wightman RM (1989) Heterogeneity of stimulated dopamine overflow within rat striatum as observed with in vivo voltammetry. Brain Res 487:311-320.

Mohebi A, Pettibone, JR, Hamid AA,Wong JMT, Vinson LT, Patriarchi T, Tian L, Kennedy RT, and Berke JD (2019) Dissociable dopamine dynamics for learning and motivation. Nature 570:65-70.

Moquin KF, Michael AC (2009) Tonic autoinhibition contributes to the heterogeneity of evoked dopamine release in the rat striatum. J Neurochem 110:1491-1501.

Moquin KF, Michael AC (2011) An inverse correlation between the apparent rate of dopamine clearance and tonic autoinhibition in subdomains of the rat striatum: a possible role of transporter-mediated dopamine efflux. J Neurochem 117:133-142. 
Noga BR, Turkson RP, Xie S, Taberner A, Pinzon A, Hentall ID (2017) Monoamine Release in the Cat Lumbar Spinal Cord during Fictive Locomotion Evoked by the Mesencephalic Locomotor Region. Front Neural Circuits 11:59.

Oh Y, Heien ML, Park C, Kang YM, Kim J, Boschen SL, Shin H, Cho HU, Blaha CD, Bennet KE, Lee HK, Jung SJ, Kim IY, Lee KH, Pyo Jang D (2018) Tracking tonic dopamine levels in vivo using multiple cyclic square wave voltammetry. Biosens Bioelectron $15: 174-182$.

Park DJ, West AR (2009) Regulation of striatal nitric oxide synthesis by local dopamine and glutamate interactions. J Neurochem 111:1457-1465.

Park C, Oh Y, Shin S, Kim J, Kang Y, Sim J, Cho HU, Lee HK, Jung SJ, Blaha CD, Bennet KE, Heien ML, Lee KK, Kim IY, Pyo Jang D (2018) Fast cyclic square-wave voltammetry to enhance neurotransmitter selectivity and sensitivity. Anal Chem 90:13348-13355.

Patel PR, Na K, Zhang H, Kozai TDY, Kotov NA, Yoon E, Chestek CA (2015) Insertion of linear $8.4 \mu \mathrm{m}$ diameter 16 channel carbon fiber electrode arrays for single unit recordings. J Neural Eng 12:046009.

Patel PR, Popov P, Caldwell CM, Welle EJ, Egert D, Pettibone JR, Roossien DH, Becker JB, Berke JD, Chestek CA (2020) High density carbon fiber arrays for chronic electrophysiology, fast scan cyclic voltammetry, and correlative anatomy. J Neural Eng 17:056029.

Patriarchi T, Cho JR, Merten K, Howe MW, Marley A, Xiong W, Folk RW, Broussard GJ, Liang R, Jang MJ, Zhong H, Dombeck D, von Zastrow M, Nimmerjahn A, Gradinaru V, William JT, Tian L (2018) Ultrafast neuronal imaging of dopamine dynamics with designed genetically encoded sensors. Science 360:eaat4422. 
Patriarchi T, Cho JR, Merten K, Marly A, Broussard GJ, Liang R, Williams J, Nimmerjahn A, von Zastrow M, Gradinaru V, Tian L (2019) Imaging neuromodulators with high spatiotemporal resolution using genetically encoded indicators. Nat Protoc 14:3471-3505

Paxinos G, Watson C (1986) The rat brain in stereotaxic coordinates. New York: Academic Press.

Phillips PEM, Stuber GD, Heien MLAV, Wightman RM, Carelli RM (2003) Subsecond dopamine release promotes cocaine seeking. Nature 422:614-618.

Puthongkham P, Venton BJ (2020) Recent advances in fast-scan cyclic voltammetry. Analyst 145:1087-1102.

Pyakurel P, Champaloux EP, Venton BJ (2016) Fast-scan cyclic voltammetry (FSCV) detection of endogenous octopamine in Drosophila melanogaster ventral nerve cord. ACS Chem Neurosci 7:1112-1119.

Resendez SL, Keyes PC, Day JJ, Hambro C, Austin CJ, Maina FK, Eidson LN, Porter-Stransky KA, Nevarez N (2016) Dopamine and opioid systems interact within the nucleus accumbens to maintain monogamous pair bonds. eLife 5:e15325.

Rice ME, Richards CD, Nedergaard S, Hounsgaard J, Nicholson C, Greenfield SA (1994) Direct monitoring of dopamine and 5-HT release in substantia nigra and ventral tegmental area in vitro. Exp Brain Res 100:395-406.

Roberts JG, Sombers LA (2018) Fast-scan cyclic voltammetry: chemical sensing in the brain and beyond. Anal Chem 90:490-504.

Robinson DL, Hermans A, Seipel AT, Wightman RM (2008) Monitoring rapid chemical communication in the brain. Chem Rev 108:2554-2584. 
Robinson DL, Zitzman DL, Williams SK (2011) Mesolimbic dopamine transients in motivated behaviors: focus on maternal behavior. Front Psychiatry 2:1-13.

Robinson DL, Wightman RM (2007) Rapid dopamine release in freely moving rats. In: Electrochemical Methods for Neuroscience (Michael AC, Borland LM, eds), pp 17-34. Boca Raton: CRC Press.

Robinson JD, Howard CD, Pastuzyn ED, Byers DL, Keefe KA, Garris PA (2014) Methamphetamine-induced neurotoxicity disrupts pharmacologically evoked dopamine transients in the dorsomedial and dorsolateral striatum. Neurotox Res 26:152-167.

Rothman RB and Baumann MH (2006) Balance between dopamine and serotonin release modulates behavioral effects of amphetamine-type drugs. Ann NY Acad Sci 1074:245260.

Sabatini BL, Tian L (2020) Imaging Neurotransmitter and Neuromodulator Dynamics In Vivo with Genetically Encoded Indicators. Neuron 108:17-32.

Salinas AG, Davis MI, Lovinger DM, Mateo Y (2016) Dopamine dynamics and cocaine sensitivity differ between striosome and matrix compartments of the striatum. Neuropharmacology 108:275-283.

Sandberg SG, Garris PA (2010) Neurochemistry of addiction: monitoring essential neurotransmitters of addiction. In: Advances in the Neuroscience of Addiction (Kuhn CM, Koob GF, eds), pp 101-136. Boca Raton, FL: CRC Press.

Saylor RA, Hersey M, West A, Buchanan AM, Berger SN, Nijhout HF, Reed MC, Best J, Hashemi P (2019) In vivo hippocampal serotonin dynamics in male and female mice: determining effects of acute escitalopram using fast scan cyclic voltammetry. Front Neurosci 13:362 
Schultz W (2007) Behavioral dopamine signals. Trends Neurosci 30:203-210.

Schuweiler DR, Howard CD, Ramsson ES, Garris PA (2018) Improving in Situ electrode calibration with principal component regression for fast-scan cyclic voltammetry. Anal Chem 90:13434-13442.

Schwerdt HN, Kim MJ, Amemori S, Homma D, Yoshida T, Shimazu H, Yerramreddy H, Karasan E, Langer R, Graybiel AM, Cima MJ (2017) Subcellular probes for neurochemical recording from multiple brain sites. Lab Chip 17: 1104-1115.

Schwerdt HN, Zhang E, Kim MJ, Yoshida T, Stanwicks L, Amemori S, Dagdeviren HE, Langer R, Cima MJ, Graybiel AM (2018) Cellular-scale probes enable stable chronic subsecond monitoring of dopamine neurochemicals in a rodent model. Commun Biol 1:144

Shi W, Pun C, Zhang X, Jones MD, Bunney BS (2000) Dual effects of D-Amphetamine on dopamine neurons mediated by dopamine and nondopamine receptors. J Neurosci 20:3504-3511.

Shin M, Field TM, Stucky CS, Furgurson MN, Johnson MA (2017) Ex vivo measurement of electrically evoked dopamine release in zebrafish whole brain. ACS Chem Neurosci 8:1880-1888.

Shu Z, Taylor IM, Michael AC (2013) The dopamine patchwork of the rat nucleus accumbens core. Eur J Neurosci 38:3221-3229.

Smith AR, Garris PA, Casto JM (2015) Real-time monitoring of electrically evoked catecholamine signals in the songbird striatum using in vivo fast-scan cyclic voltammetry. J Chem Neuro 66-67:28-39.

Srejic LR, Hamani C, Hutchison WD (2015) High-frequency stimulation of the medial prefrontal cortex decreases cellular firing in the dorsal raphe. Eur J Neurosci 41:1219-1226. 
Stagkourakis S, Dunevall J, Taleat Z, Ewing AG, Broberger C (2019) Dopamine release dynamics in the tuberinfundibular dopamine system. J Neurosci 39:4009-4022.

Sulzer D, Sonders MS, Poulsen NW, Galli A (2005) Mechanisms of neurotransmitter release by amphetamines: a review. Prog Neurobiol 75:406-433.

Sun F, Zeng J, Jing M, Zhou J, Feng J, Owen SF, Luo Y, Li F, Wang H, Yamaguchi T, Yong Z, Gao Y, Peng W, Wang L, Zhang S, Du J, Lin D, Xu M, Kreitzer AC, Cui G, Li y (2018) A Genetically Encoded Fluorescent Sensor Enables Rapid and Specific Detection of Dopamine in Flies, Fish, and Mice. Cell 12:481-496.

Tan C, Robbins EM, Wu B, Cui XT (2021) Recent advances in in vivo neurochemical monitoring. Micromachines 12:1-32.

Taylor IM, Jaquins-Gerstl A, Sesack SR, Michael AC (2012) Domain-dependent effects of DAT inhibition in the rat dorsal striatum. J Neurochem 122:283-294.

Taylor IM, Nesbitt KM, Walters SH, Varner EL, Shu Z, Bartlow KM, Jaquins-Gerstl AS, Michael AC (2015) Kinetic diversity of dopamine transmission in the dorsal striatum. J Neurochem 133:522-531.

Venton BJ, Wightman RM (2003) Psychoanalytical Electrochemistry: Dopamine and Behavior. Anal Chem 75: 414A-421A

Venton BJ, Michael DJ, Wightman RM (2003) Correlation of local changes in extracellular oxygen and $\mathrm{pH}$ that accompany dopaminergic terminal activity in the rat caudateputamen. J Neurochem 84:373-381.

Venton BJ, Cao Q (2020) Fundamentals of fast-scan cyclic voltammetry for dopamine detection. Analyst 145:1158-1168 
Walters SH, Taylor IM, Shu Z, Michael AC (2014) A novel restricted diffusion model of evoked dopamine. ACS Chem Neurosci 5:776-783.

Walters SH, Robbins EM, Michael AC (2015) Modeling the kinetic diversity of dopamine in the dorsal striatum. ACS Chem Neurosci 6:1468-1475.

Walters SH, Shu Z, Michael AC, Levitan ES (2020) Regional Variation in Striatal Dopamine Spillover and Release Plasticity. ACS Chem Neurosci 11:888-899.

Wang H, Jing M, Li Y (2018) Lighting up the brain: genetically-encoded fluorescent sensors for imaging neurotransmitters and neuromodulators. Curr Opin Neurobiol 50:171-178.

Watson CJ, Venton BJ, Kennedy RT (2006) In vivo measurements of neurotransmitters by microdialysis sampling. Anal Chem 78: 1391-1399.

Wightman RM, Amatore C, Engstrom RC, Hale PD, Kristensen EW, Kuhr WG, May LJ (1988) Real-time characterization of dopamine overflow and uptake in the rat striatum. Neuroscience 25:513-523.

Wightman RM, Heien ML, Wassum KM, Sombers LA, Aragona BJ, Khan AS, Ariansen JL, Cheer JF, Phillips PEM, and Carelli RM (2007) Dopamine release is heterogeneous within microenvironments of the rat nucleus accumbens. Eur J Neuro 26: 2046-2054

Wu Q, Reith ME, Wightman RM, Kawagoe KT, Garris PA (2001) Determination of release and uptake parameters from electrically evoked dopamine dynamics measured by real-time voltammetry. J Neurosci Methods 112:119-133.

Yocky AG and Covey DP (2020) Evolution of in vivo dopamine monitoring techniques. Pharm Biochem Beh 200:173078 
Zachek MK, Takmakov P, Moody B, Wightman RM, McCarty GS (2009) Simultaneous decoupled detection of dopamine and oxygen using pyrolyzed carbon microarrays and fastscan cyclic voltammetry. Anal Chem 81:6258-6265

Zachek MK, Takmakov P, Park J, Wightman RM, McCarty GS (2010) Simultaneous monitoring of dopamine concentration at spatially different brain locations in vivo. Biosens Bioelectron 25:1179-1185 


\section{APPENDIX: FIGURES}

Figure 1: Representative sensors and system components

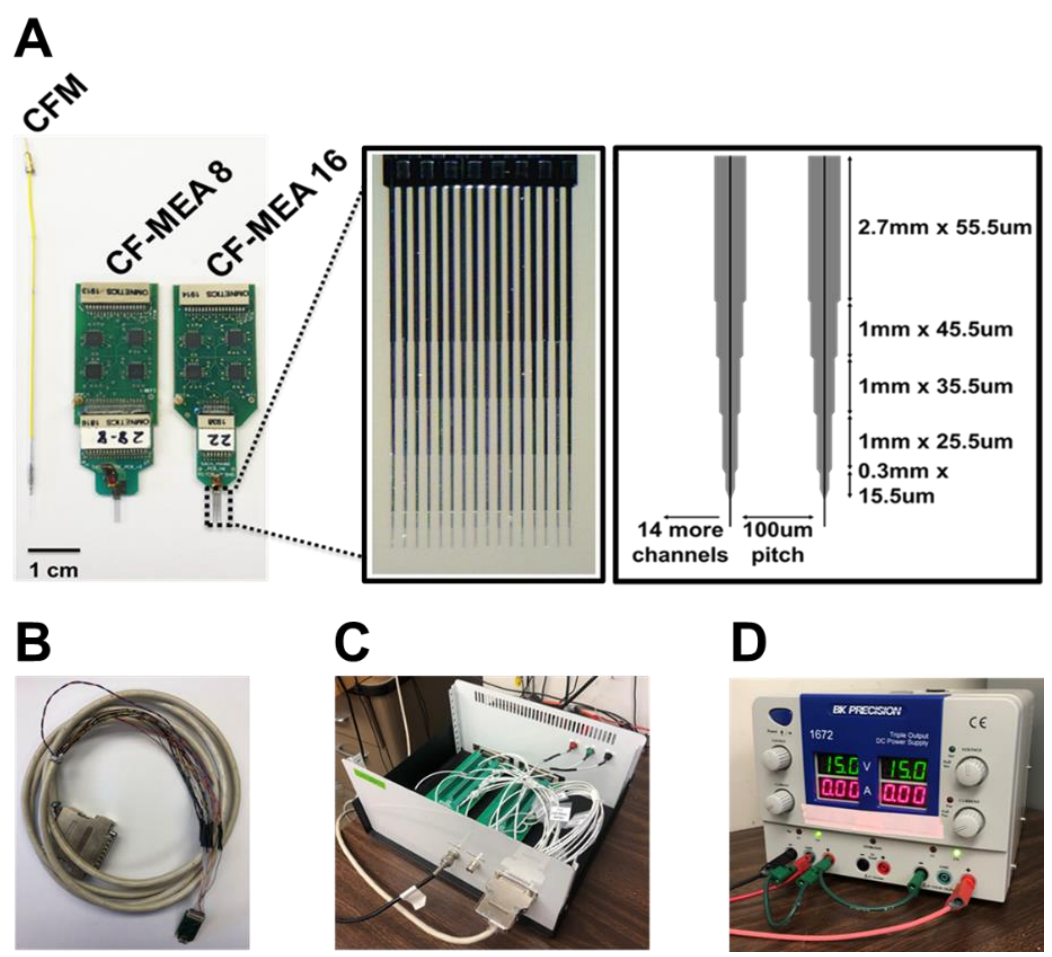

Figure 1. Representative sensors and system components. A. representative CFM, CF-MEA 8, and CF-MEA-16 sensors are shown at left. The CFM has a single carbon fiber $(\sim 7 \mu \mathrm{m}$ diameter, not visible) trimmed to a length of $50 \mu \mathrm{m}$. The borosilicate capillary tube is sealed with epoxy, and electrical connection is established by melting bismuth alloy to secure a copper wire (yellow). A gold pin is soldered in place to facilitate coupling with the headstage. CF-MEA 8 and CF-MEA16 are shown connected via an Omnetics coupler to a headstage (headstage is also shown connected to the lead cable in B). INSETS depict the silicone support structure present in the CFMEA-16. The silicone support reduces in size concentrically as it becomes more distal to the Omnetics connection. The lead cable in $\mathbf{B}$ is connected to the breakout box depicted in $\mathbf{C}$, and the breakout box is powered by a standalone power supply shown in $\mathbf{D}$. 
Figure 2: Flow Injection Apparatus Schematic

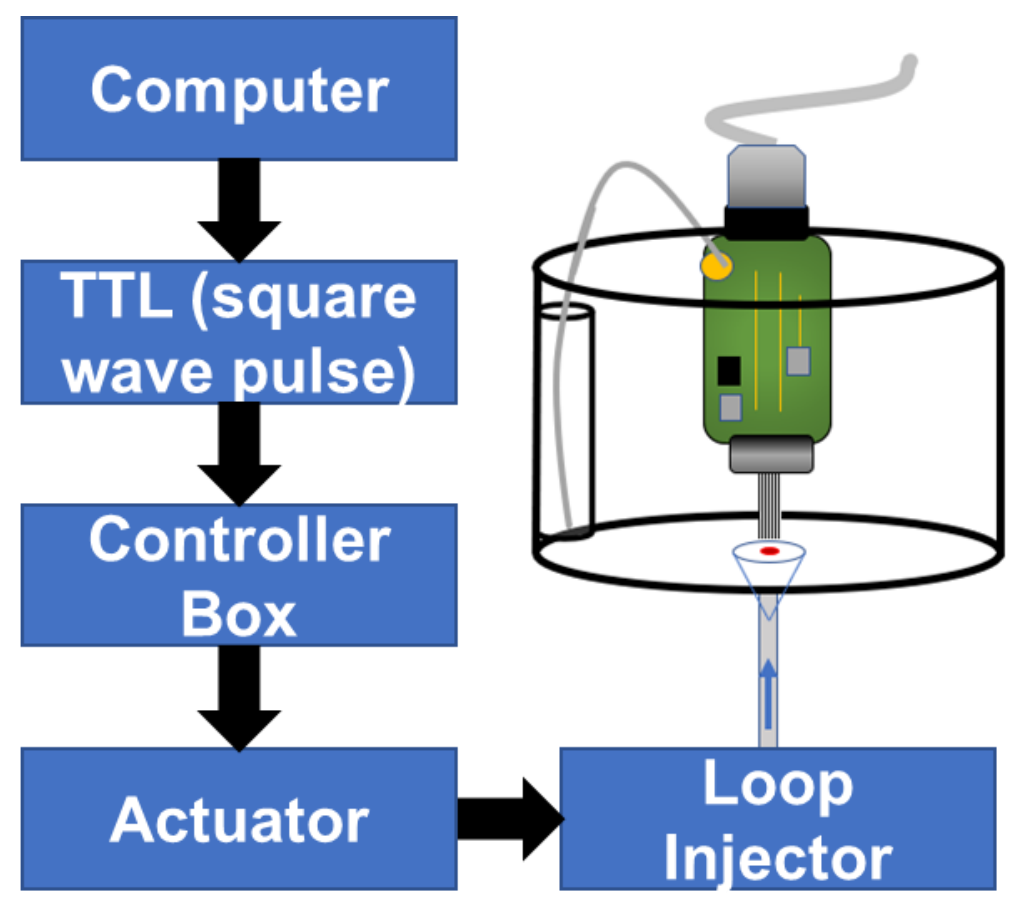

Figure 2: Flow Injection Apparatus Schematic. Components of the flow injection apparatus are depicted including the computer, TTL pulse, controller box, actuator, loop injector, and buffer reservoir. A known concentration of DA is injected via syringe into the loop injector. Tris buffer is continually injected into the reservoir at a user-defined rate. When generated by the computer, the TTL pulse triggers a nitrogen-driven actuator to switch injected solution from Tris buffer to a bolus of DA-containing buffer. 
Figure 3. Flow Injection Interface Placement by Sensor Type
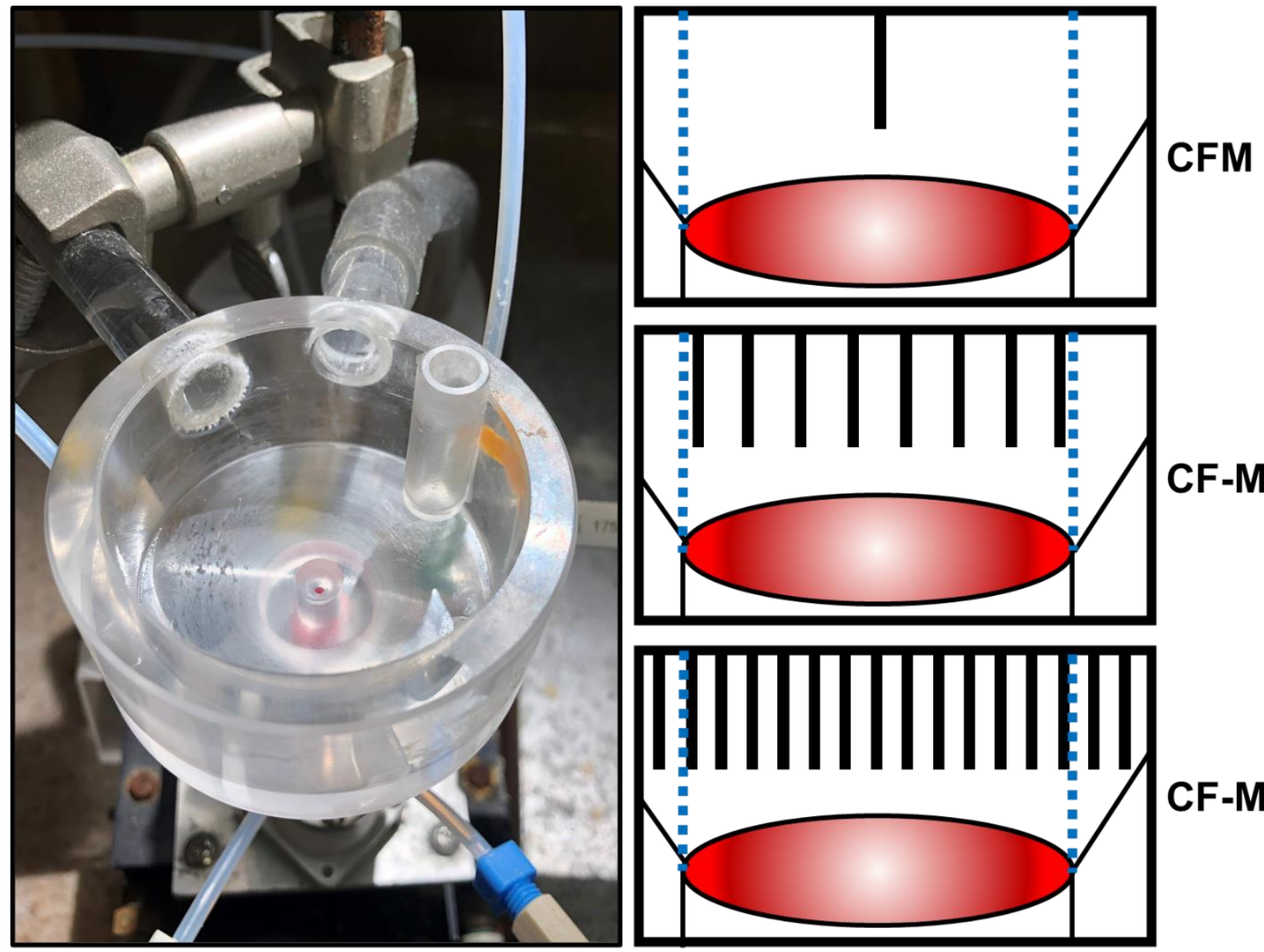

CF-MEA-8

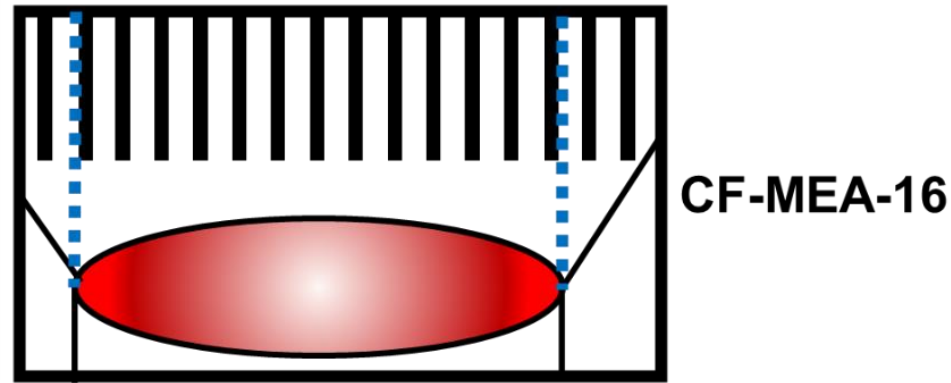

Figure 3. Flow Injection Interface Placement by Sensor Type. A photograph depicting the FIA apparatus reservoir and outlet is shown at left. A conceptual rendering of the sensing surface(s) of a CFM (top right), CF-MEA-8 (middle right), and CF-MEA-16 (bottom right) is shown to highlight considerations associated with CF-MEA-8 and CF-MEA-16 dorsoventral and mediolateral placement at the tubing outlet of the FIA apparatus. 
Figure 4: FIA Characterization of MEA-8

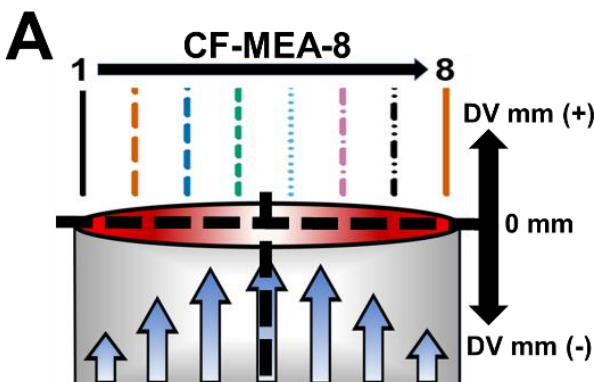

$M L \mathrm{~mm}(+) \underset{ }{0} \mathrm{~mL}$ mm (-)

B

DV $0.0 \mathrm{~mm}$

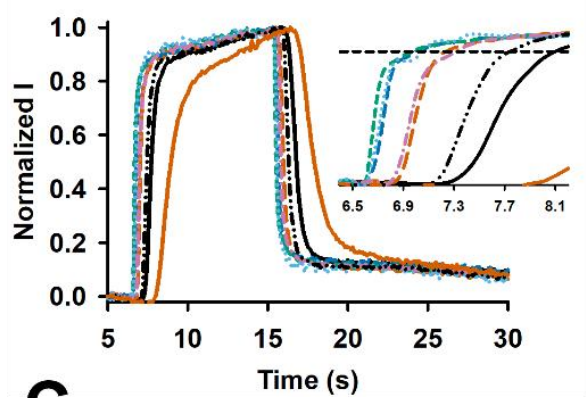

C $\mathrm{DV}+0.5 \mathrm{~mm}$

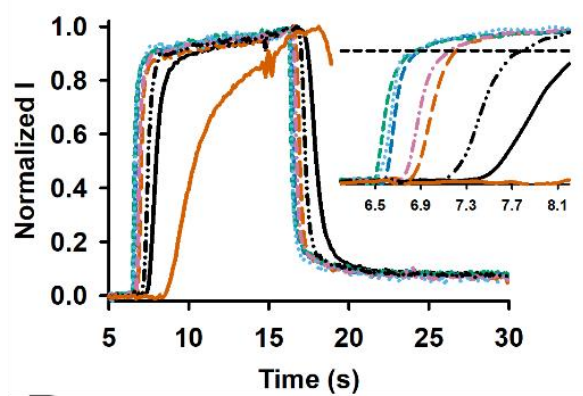

D $\mathrm{DV}+1.0 \mathrm{~mm}$

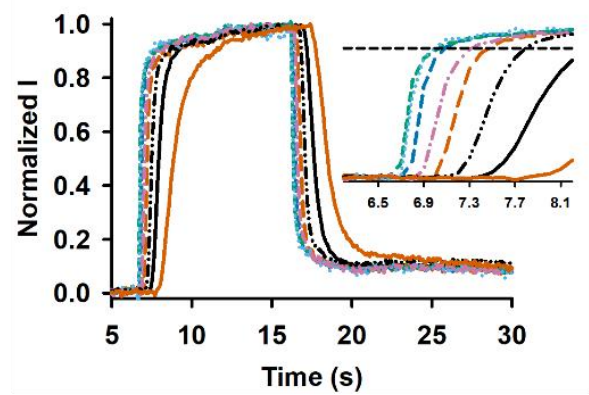

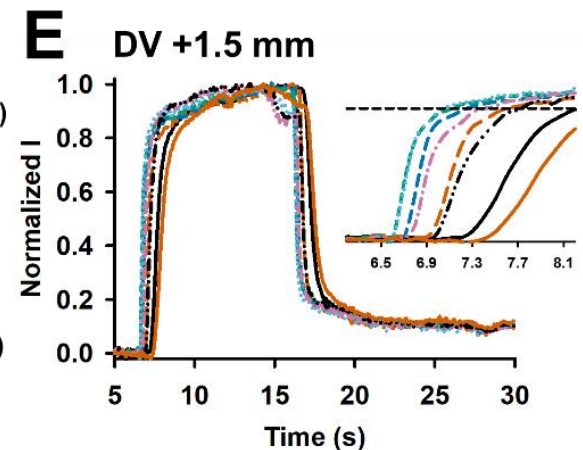

$\mathbf{F}$
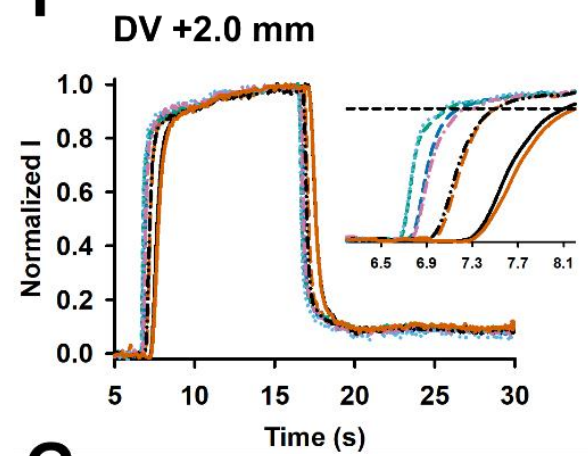

G

DV $+2.5 \mathrm{~mm}$

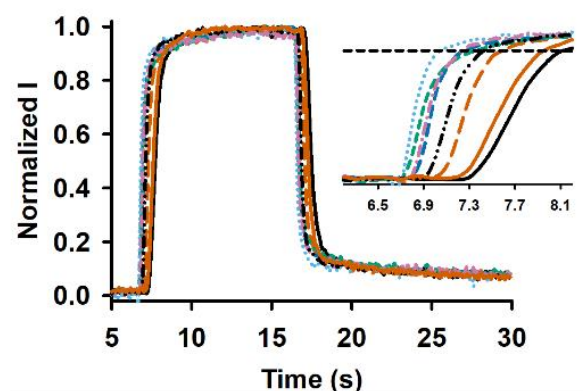

H Fastest site

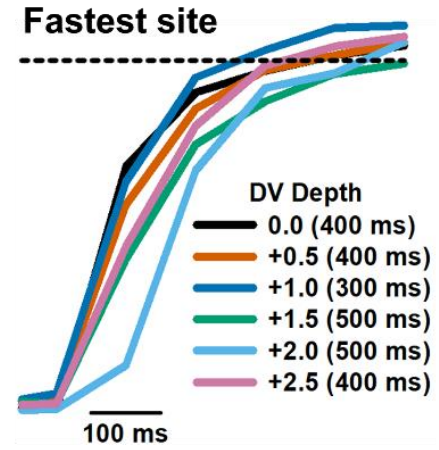

$M L 0.0 \mathrm{~mm}$

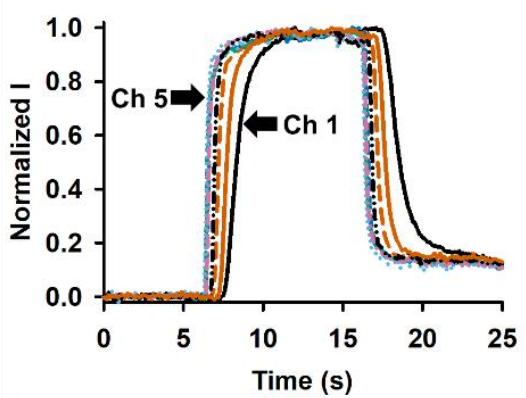

J

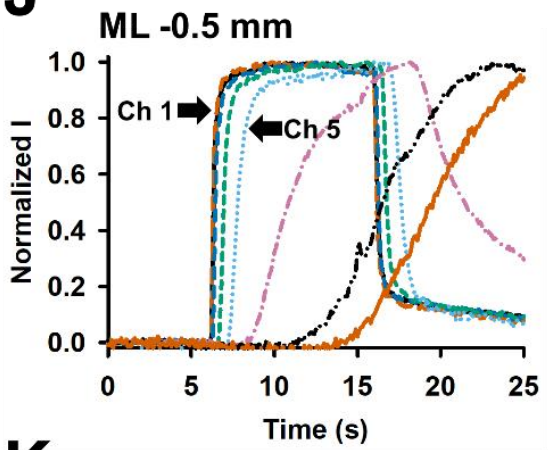

K
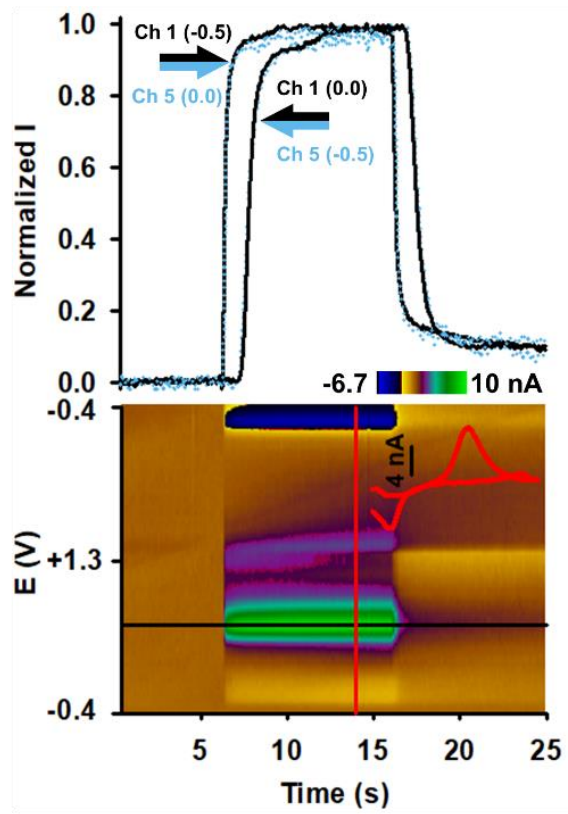
Figure 4. FIA Characterization of MEA-8. A. A conceptual rendering of the measurement of the leading edge of a DA bolus by the CF-MEA-8 is shown. The position of the CF-MEA-8 was adjusted in the mediolateral (ML) and dorsoventral (DV) planes for measurements described in subsequent panels. Color key for recording site applies to panels $\mathbf{B}$ to $\mathbf{G}$. Temporal responses to the DA bolus and normalized to maximal current are shown for the CF-MEA- 8 centered $(\mathrm{ML}=0$ $\mathrm{mm}$ ) above the tubing outlet at a DV position $0.0(\mathbf{B}),+0.5(\mathbf{C}),+1.0(\mathbf{D}),+1.5(\mathbf{E}),+2.0(\mathbf{F})$, and $+2.5(\mathbf{G}) \mathrm{mm}$. INSETs show an expanded view of the response to the leading edge of the DA bolus. H. The fastest response at each DV position is shown. Distance is shown by the adjacent color key. The response collected at DV $+1.0 \mathrm{~mm}$ is as fast if not faster than all other responses. This distance is thus suitable for assessing response time and provides a safe distance above the outlet to position CF-MEAs. Also notice how responses recorded by the lateral sites are more distorted at closer distances compared to far, perhaps due to turbulent flow as the buffer stream leaves the tubing, enters the reservoir, and interacts with reservoir sides. Dashed line in $\mathbf{B}$ to $\mathbf{H}$ is $85 \%$ of maximal current. Temporal responses to the DA bolus and normalized to maximal current are shown for a CF-MEA-8 centered above the tubing outlet $(\mathbf{I} ; \mathrm{ML}=0 \mathrm{~mm})$ and offset by $0.5 \mathrm{~mm}$ $(\mathbf{J} ; \mathrm{ML}=-0.5 \mathrm{~mm})$. Line color follows key in $\mathbf{A}$. The fastest response in $\mathbf{I}$, site 5, is no longer the fastest response in $\mathbf{J}$ when moved away the center of the tubing outlet. Conversely, the slowest response in $\mathbf{I}$, site 1, becomes the fastest response in $\mathbf{J}$ when it is centered above the tubing outlet. Line color follows key in $\mathbf{A}$. K. Responses for sites 1 and 5 in $\mathbf{I}$ and $\mathbf{J}$ are reproduced for clarity (top). Note how well responses from sites 1 and 5 overlap when positioned similarly (within $\approx 160$ $\mu \mathrm{m}$, the distance between sites), above the tubing outlet. The pseudocolor plot and individual voltammogram for the response described by the solid black line (site 1 , ML $-0.5 \mathrm{~mm}$; bottom) indicate that DA is the measured analyte. Horizontal black line of the pseudocolor plot delineates 
current measured at the peak oxidative potential for DA. Vertical dashed red line delineates the location of the individual background-subtracted cyclic voltammogram shown in red. Collectively, results shown in $\mathbf{I}$ to $\mathbf{K}$ demonstrate that the temporal response is more related to position in the measurement field than along the CF-MEA-8. All temporal responses recorded a 10-s, 1- $\mu$ M DA bolus and are single recordings. 
Figure 5: Laminar flow of a DA bolus with FIA
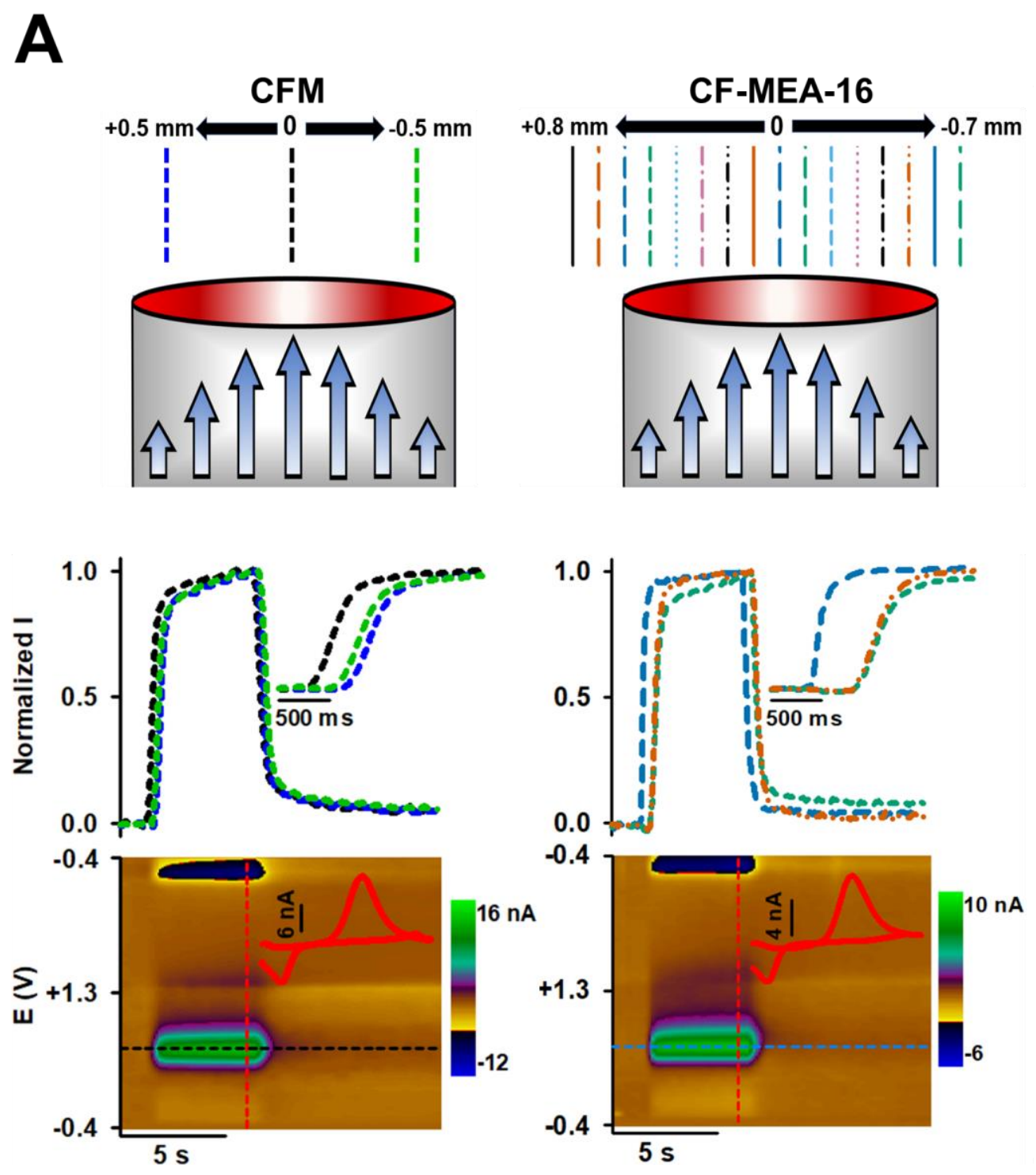


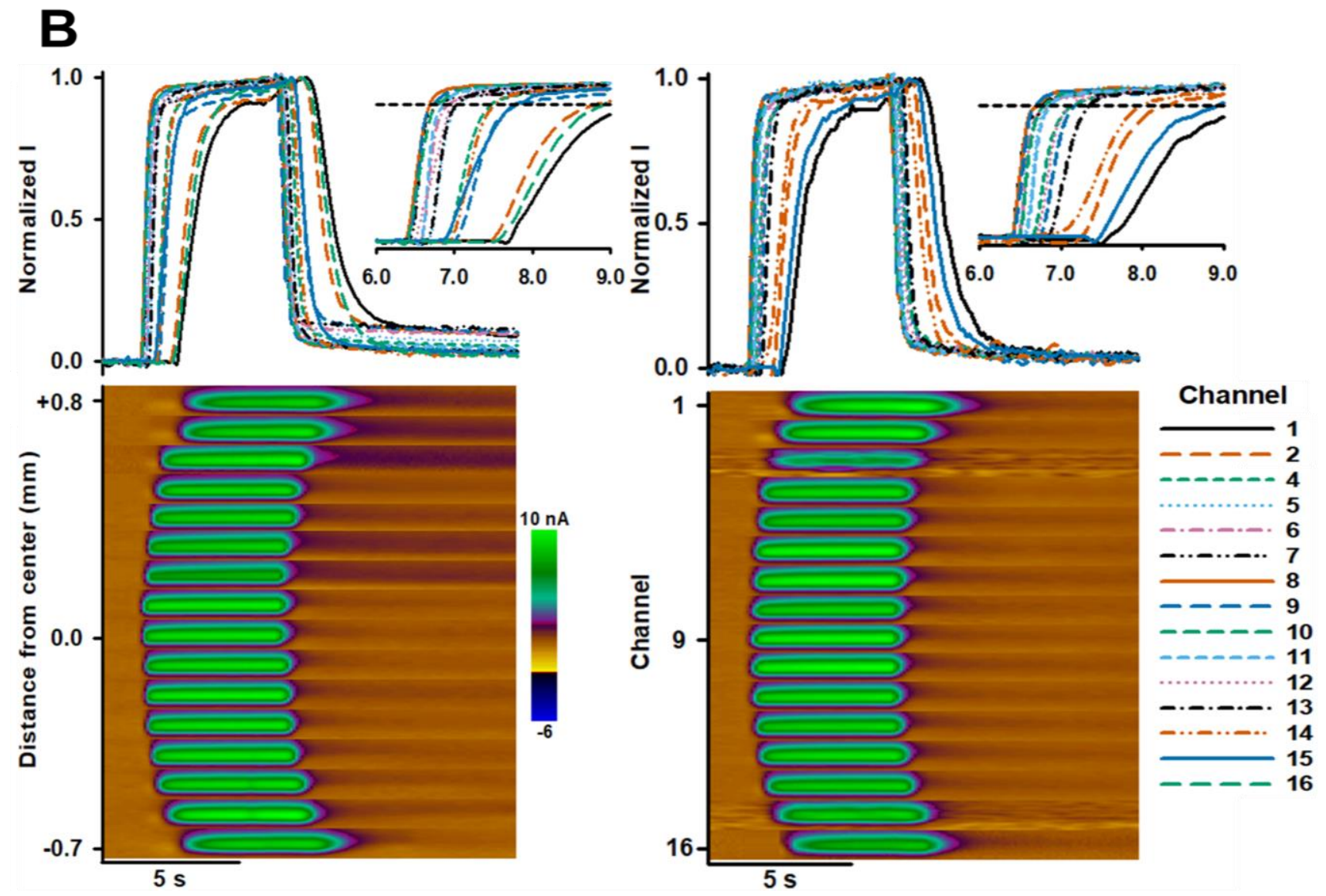

Figure 5. Laminar flow of a DA bolus with FIA. A. CFM and CF-MEA-16 FSCV measurements are shown to the left and right, respectively. Top is a conceptual rendering of the leading edge of a DA bolus as it arrives at the reservoir opening (1.2 mm). The CFM (top left) or CF-MEA-16 (top right) is placed $1 \mathrm{~mm}$ above the opening and positioned at different mediolateral locations. After entering buffer flow ( $4 \mathrm{ml} / \mathrm{min})$ at the injection port, the leading edge of the DA bolus is distorted as it travels the length $(\approx 6 \mathrm{~cm})$ of tubing to the reservoir due to slower velocities of laminar flow nearest the tubing wall resulting from drag. Thus, the leading edge of the DA solution arrives faster in the tubing center versus the edges. Left (middle) shows recordings of the DA bolus by a CFM either placed in the center $(0 \mathrm{~mm})$ of the opening or $0.5 \mathrm{~mm}$ to either side $( \pm)$. Right (middle) shows recordings of the DA bolus by center site 9 of the MEA-16 when placed at similar locations. 
INSETs show the initial response to the DA bolus. Line color follows the key in top. Bottom shows pseudocolor plots (time - $\mathrm{x}$ axis, applied potential - $\mathrm{y}$ axis, measured current $-\mathrm{z}$ axis) displaying all background-subtracted voltammograms serially in time that generated the dashed black (left) and dashed blue (right) temporal responses above. Horizontal dashed lines delineate current of the temporal responses measured at the peak oxidative potential for DA. Vertical dashed red lines delineate the location of the individual background-subtracted cyclic voltammograms shown in red (applied potential - $\mathrm{x}$ axis, measured current - y axis) in the INSETS. B. Responses to a DA bolus were recorded by center site 9 of a MEA-16 as it is positioned at $100-\mu \mathrm{m}$ increments across the tubing outlet (left; line color follows the key in $\mathbf{A}$, top right.) and simultaneously at each site of the same MEA-16 (right; line color follows adjacent key). Temporal responses are expanded in INSETs (top; dashed line is 85\% maximal response). Pseudocolor plots for each recording site show the portion of the applied potential ( +0.28 to $0.96 \mathrm{~V})$ canvasing DA oxidation (bottom). All temporal responses recorded a 5-s, 1- $\mu \mathrm{M}$ DA bolus, are normalized to maximal current, and are the average of 3 replicates. 
Figure 6: Individually centered response times
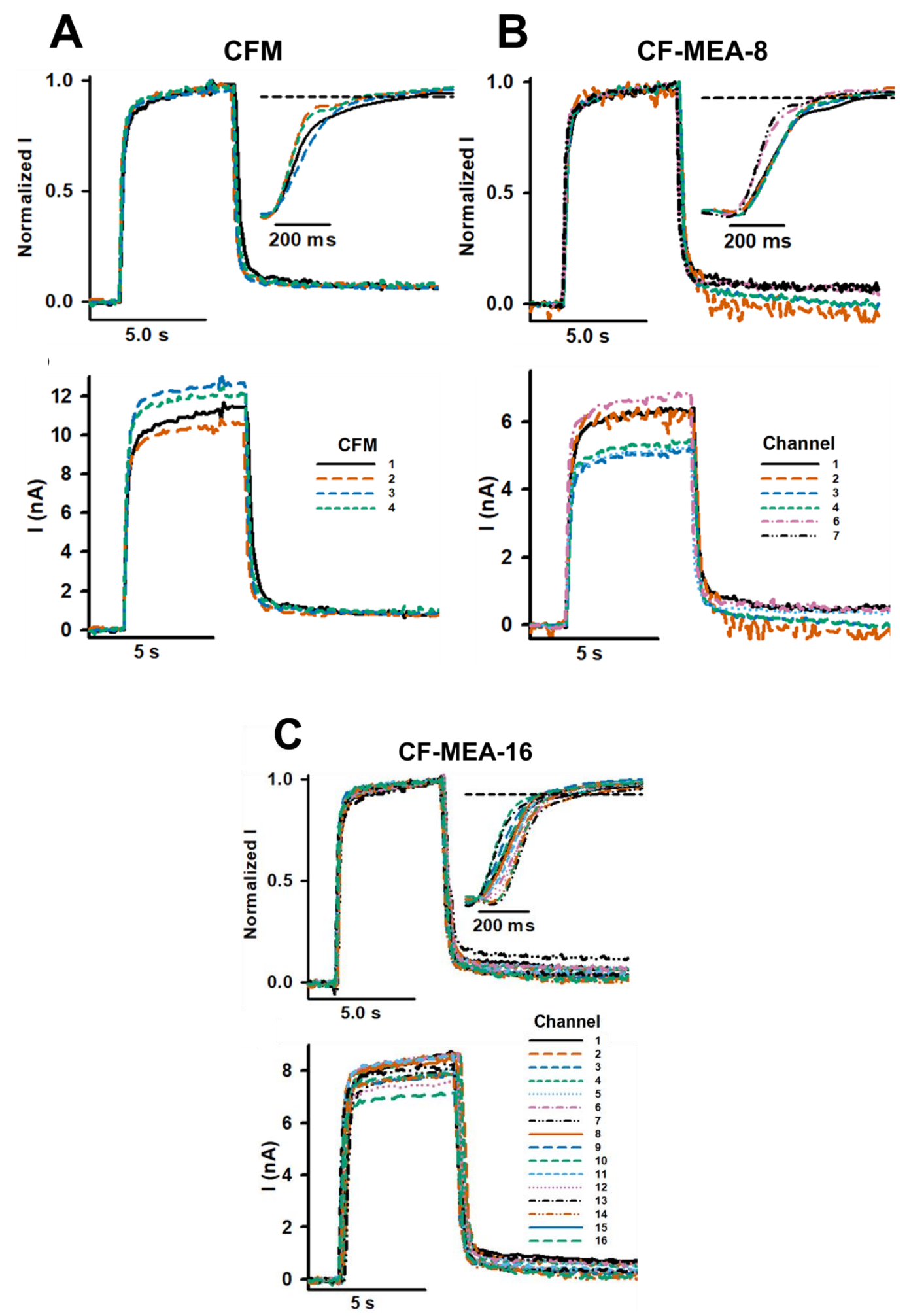
Figure 6: Individually centered response times. Individually centered temporal responses to a 5-s DA bolus normalized to maximal current (top) or in absolute current (bottom) are shown for CFMs (A), and each site of a CF-MEA-8 (B) and CF-MEA-16 (C). INSETs show expanded view of the response to the leading edge of the DA bolus. CFMs and arrays were centered $(\mathrm{ML}=0 \mathrm{~mm})$ above the tubing outlet at DV position of $+1.0 \mathrm{~mm}$. ML position of the CF-MEA- 8 was adjusted in increments of $160 \mu \mathrm{m}$, the distance between sites, to center individual sites. ML position of the CF-MEA-16 was adjusted in increments of $100 \mu \mathrm{m}$, the distance between sites, to center individual sites. Dashed line is $85 \%$ maximum. 
Figure 7: Statistical analysis of individually centered temporal responses

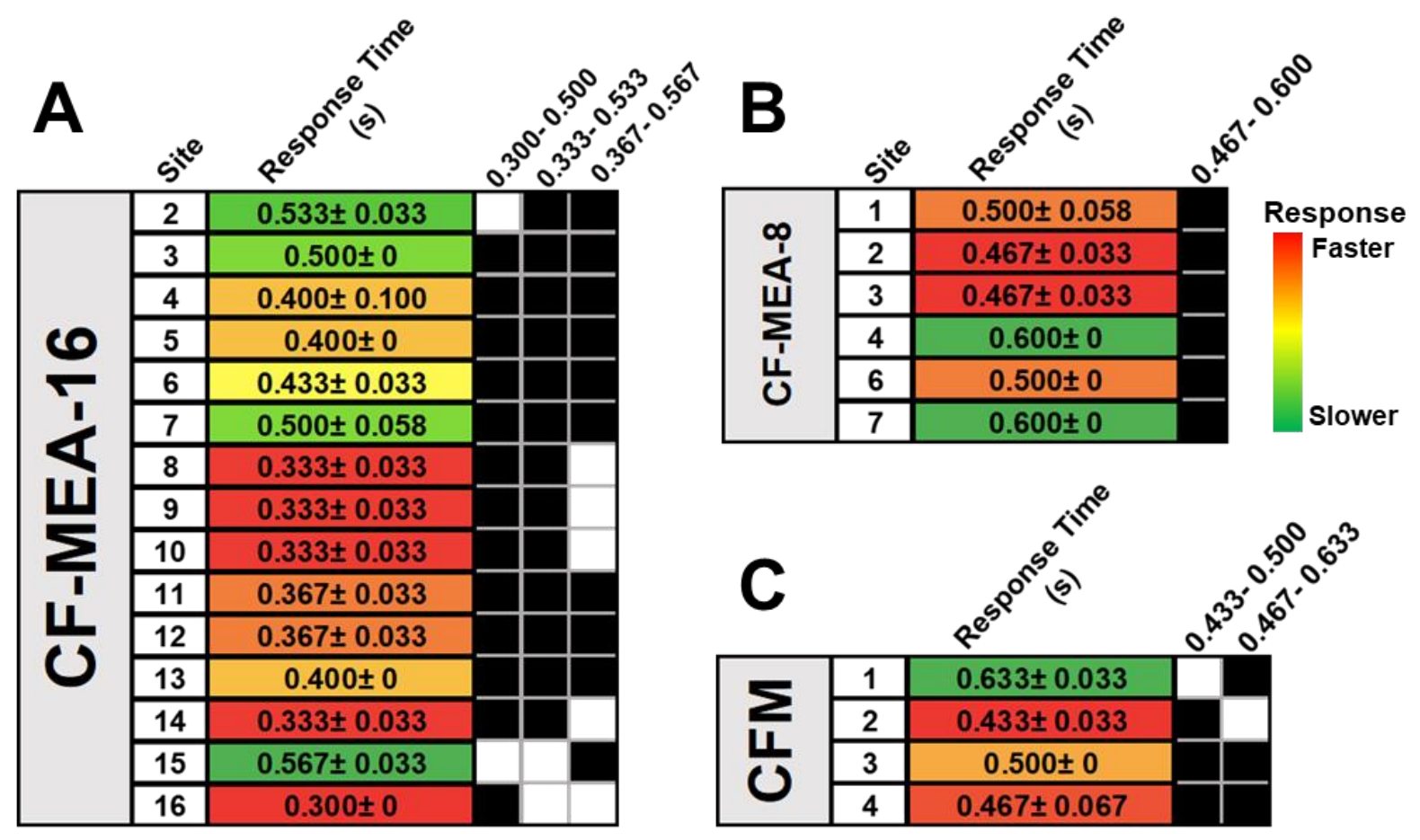

D

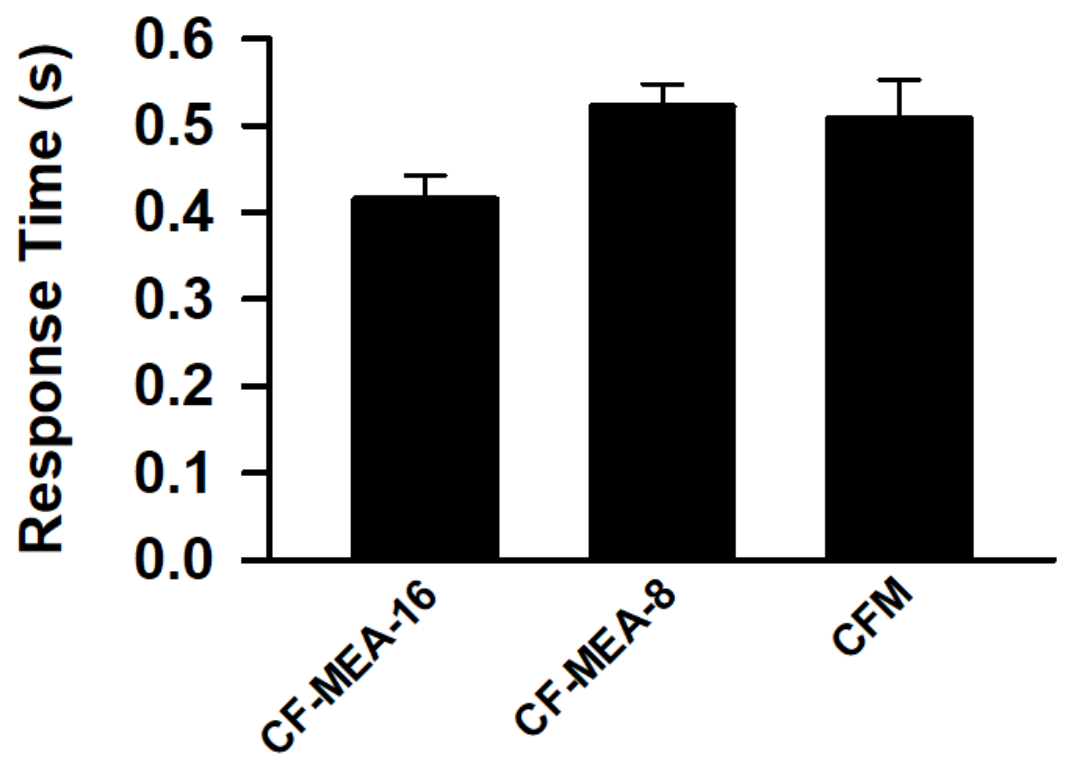


Figure 7: Statistical analysis of individually centered temporal responses. A nested one-way ANOVA was performed on individually centered response times for a CF-MEA-16 (A) and CFMEA-8 (B), and a one-way ANOVA was performed on individually centered response times for CFMs (C). Temporal response to a 5-s, 1- $\mu \mathrm{M}$ DA bolus was determined as time to $85 \%$ of maximal normalized current for CFMs and sites that were individually centered $(\mathrm{ML}=0 \mathrm{~mm}) 1-\mathrm{mm}$ above the tubing outlet. A total of three replicates were collected for each site or CFM; data are mean $\pm \mathrm{SEM}$ of these replicates. Color scale represents response time relative to each sensor type. Columns with solid black boxes are Tukey-Kramer groupings determined from post-hoc analysis. A solid black box within a grouping indicates that values are not significantly different from each other or conversely, that they are significantly different from values indicated by an open box. A one-way ANOVA was performed on response time across the CF-MEA-16, CF-MEA-8, and CFM (D). Data are mean \pm SEM of each site of the arrays or CFM after replicate averaging. No significant effect of sensor type on individually centered response time was found. 
Figure 8: Statistical analysis of group-centered response times

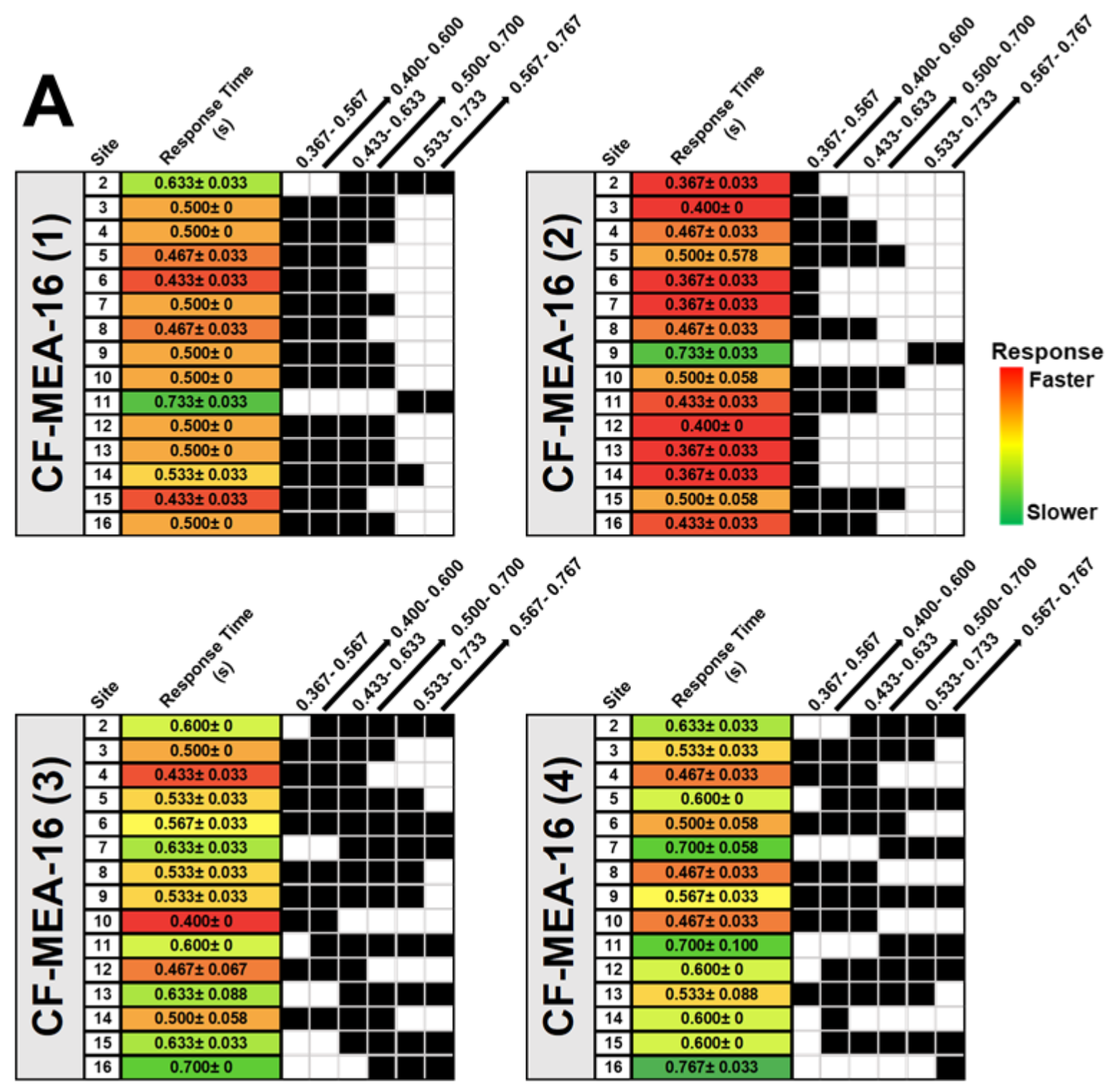




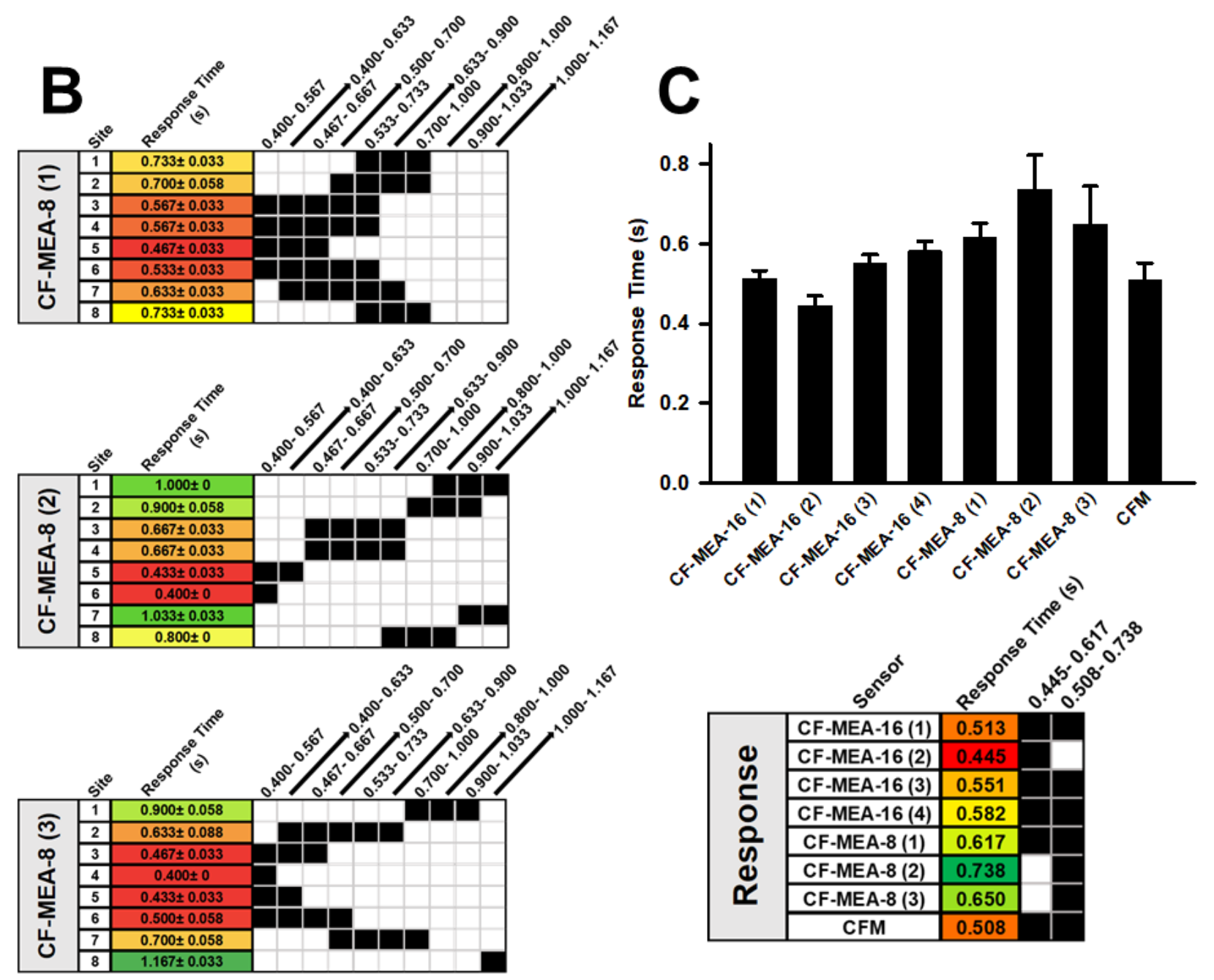

Figure 8: Statistical analysis of group-centered response times. A nested one-way ANOVA was performed on group-centered response times for CF-MEA-16s (A) and CF-MEA-8s (B). Response time was determined as in Figure 6, except that the position of the CF-MEA-16 was adjusted only twice, centering between sites 1 to 8 or 9 to 16 for two sets of 8 simultaneous recordings. The position of the CF-MEA-8 was not moved; thus, all 8 sites were simultaneously recorded. A total of three replicates were collected for each site; data are mean \pm SEM of these replicates. A one-way ANOVA was performed on response time across CF-MEA-16s, CF-MEA8s, and CFMs (C). Data are mean \pm SEM of each site of the arrays or CFM (from Figure 6D). Post- 
hoc Tukey-Kramer analysis is depicted by statistical groupings as in Figure 7. Color scale represents response time relative to each sensor type in $\mathbf{A}$ and $\mathbf{B}$ and relative to sensor in $\mathbf{C}$. 
Figure 9: Comparison of calibration curves and peak oxidative electrochemistry
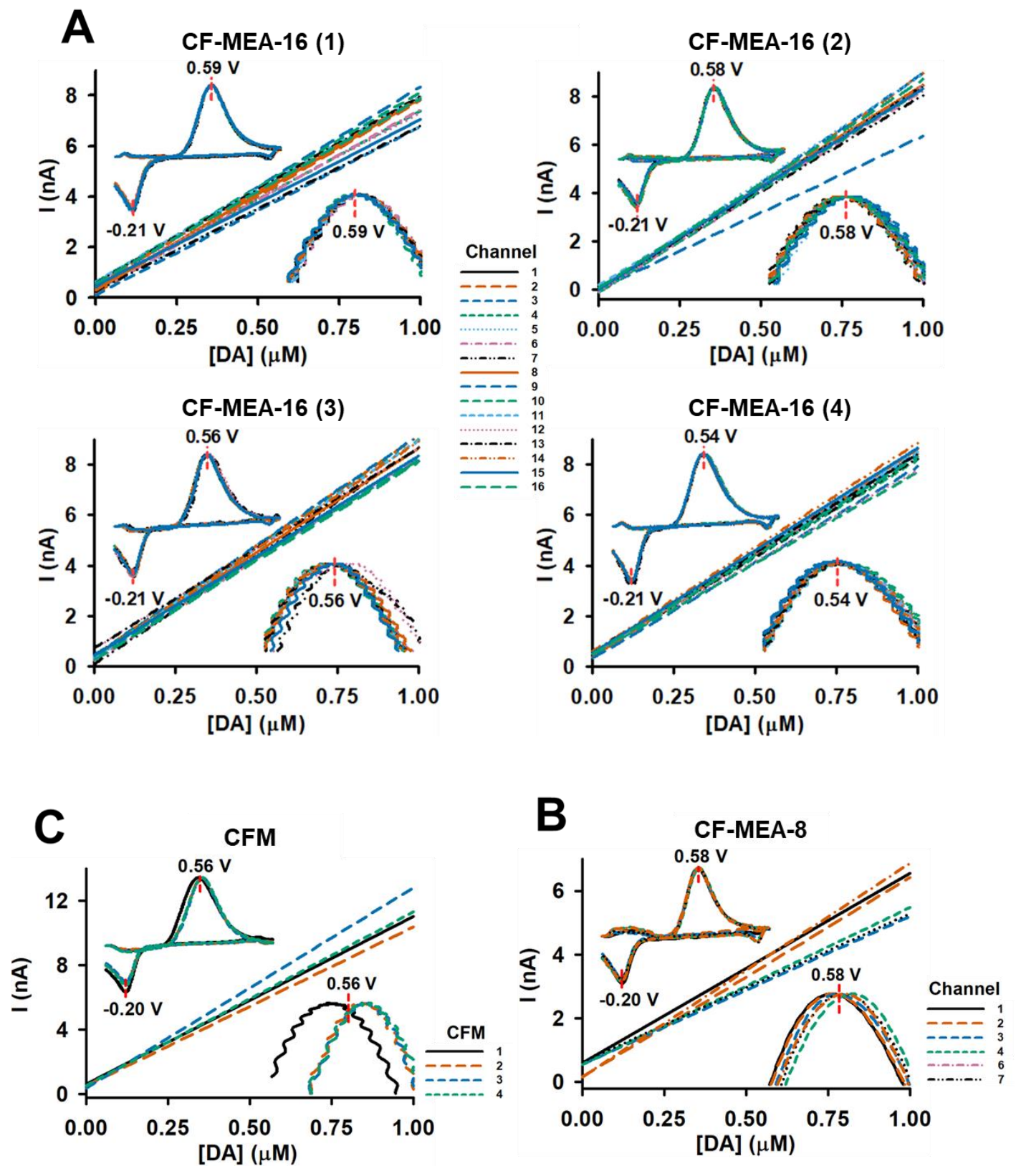
Figure 9: Comparison of calibration curves and peak oxidative electrochemistry. Calibration curves and background-subtracted cyclic voltammograms are shown for four CF-MEA-16s (A), a CF-MEA-8 (B), and four CFMs (C). Calibration curves are the best-fit line to maximal current obtained from five DA concentrations (0-, 0.25-, 0.5-, 0.75-, and 1- $\mu \mathrm{M}$; average of three replicates) delivered as a 5-s bolus. Sensors were centered and positioned $1 \mathrm{~mm}$ above the tubing outlet. Top INSETS show overlays of voltammograms collected from the $1-\mu \mathrm{M}$ DA bolus. Average peak oxidative and reductive potentials for DA are identified and depicted by dashed red vertical lines. Bottom INSETs expand the current canvassing peak oxidative potential. 
Figure 10: Statistical analysis of sensitivity
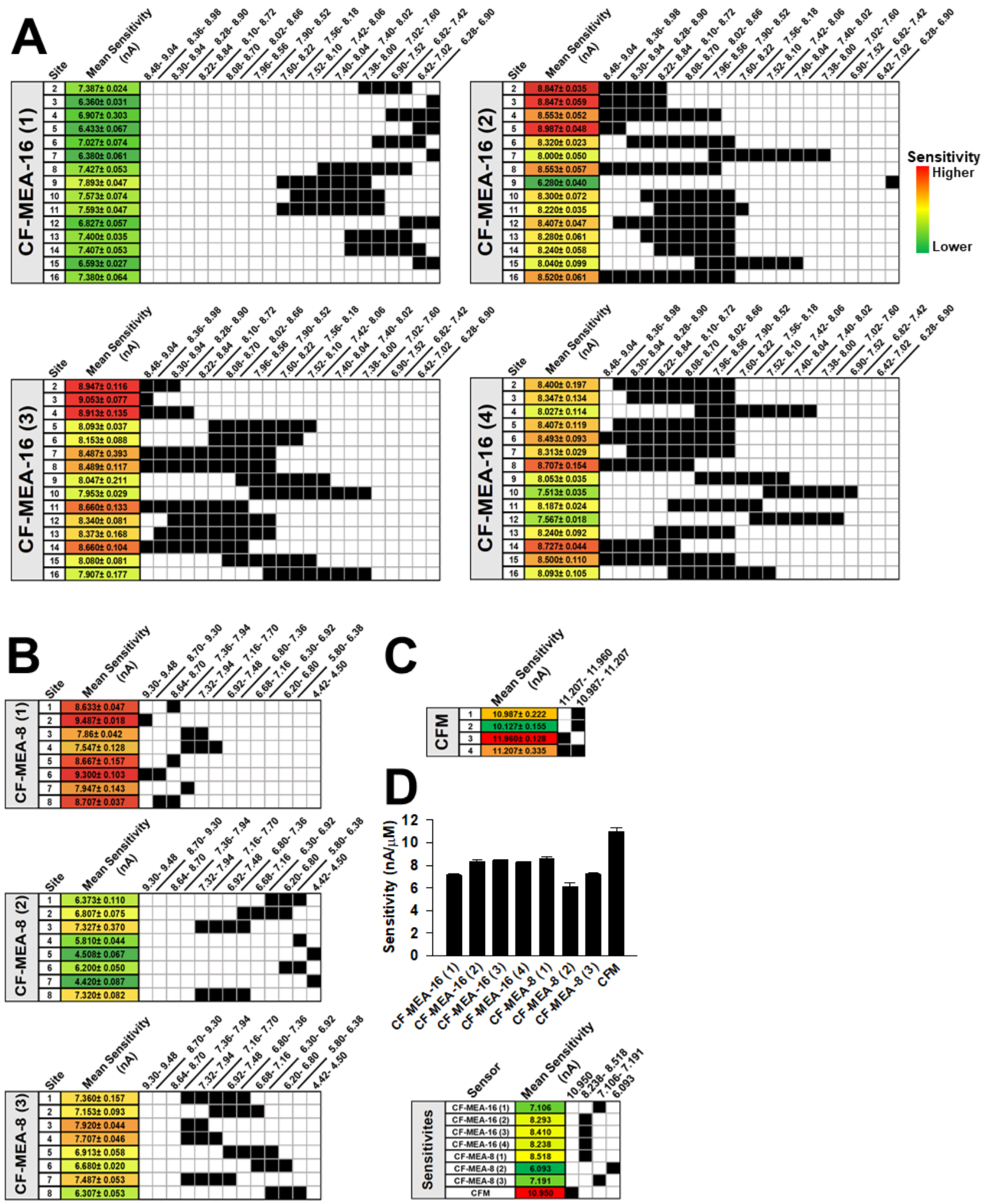
Figure 10: Statistical analysis of sensitivity. A nested one-way ANOVA was performed on sensitivity for CF-MEA-16s (A) and CF-MEA-8s (B), and a one-way ANOVA was performed on sensitivity for CFMs (C). A total of three replicates were analyzed for each site or CFM; data are mean \pm SEM of these replicates. A one-way ANOVA was performed on sensitivity across CFMEA-16s, CF-MEA-8s, and CFMs (D). Data are mean+SEM of each site of the arrays or CFM after replicate averaging. Post-hoc Tukey-Kramer analysis is depicted by statistical groupings as in Figure 7. Color scale represents response time relative to each sensor type in $\mathbf{A}, \mathbf{B}$, and $\mathbf{C}$ and relative to sensor in $\mathbf{D}$. 
Figure 11: Statistical analysis of peak oxidative potential for DA
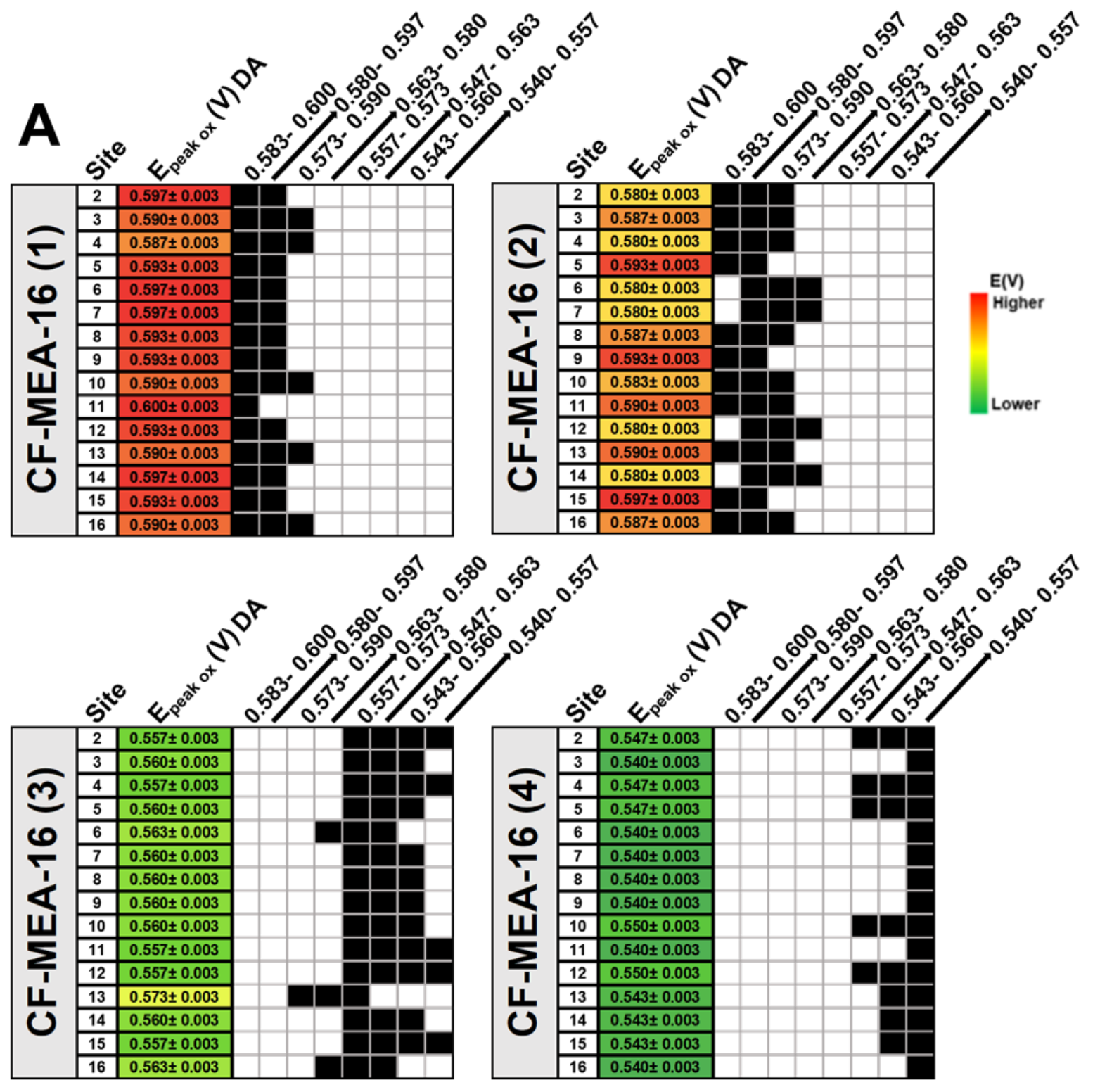


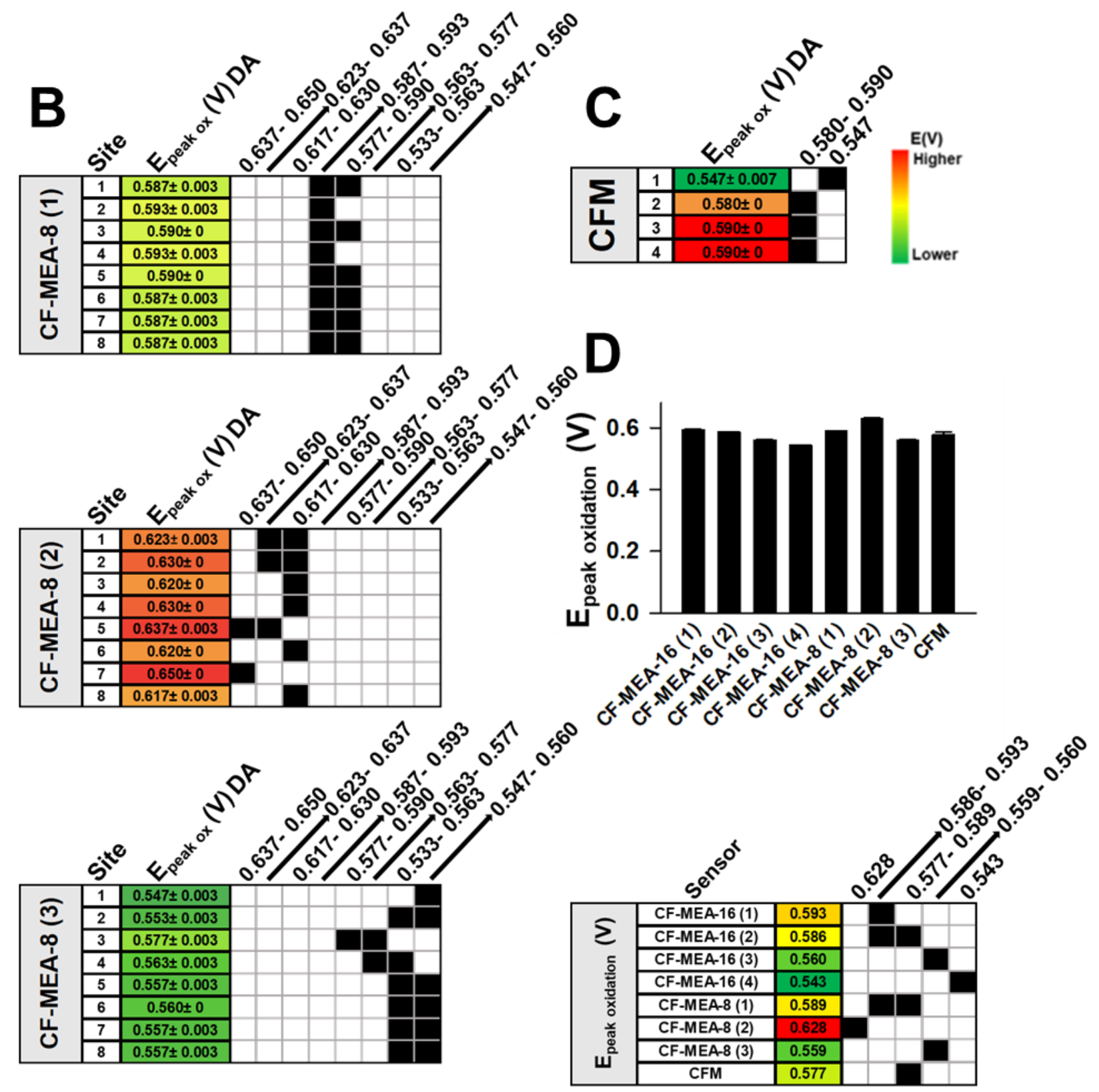

Figure 11. Statistical analysis of peak oxidative potential for DA. A nested one-way ANOVA was performed on peak oxidative potential for CF-MEA-16s (A) and CF-MEA-8s (B), and a oneway ANOVA was performed on peak oxidative potential for CFMs (C). A total of three replicates were analyzed for each site or CFM; data are mean \pm SEM of these replicates. A one-way ANOVA was performed on peak oxidative potential across CF-MEA-16s, CF-MEA-8s, and CFMs (D). 
Data are mean \pm SEM of each site of the arrays or CFM after replicate averaging. Post-hoc TukeyKramer analysis is depicted by statistical groupings as in Figure 7. Color scale represents response time relative to each sensor type in $\mathbf{A}, \mathbf{B}$, and $\mathbf{C}$ and relative to sensor in $\mathbf{D}$. 
Figure 12. Statistical analysis of RMS noise

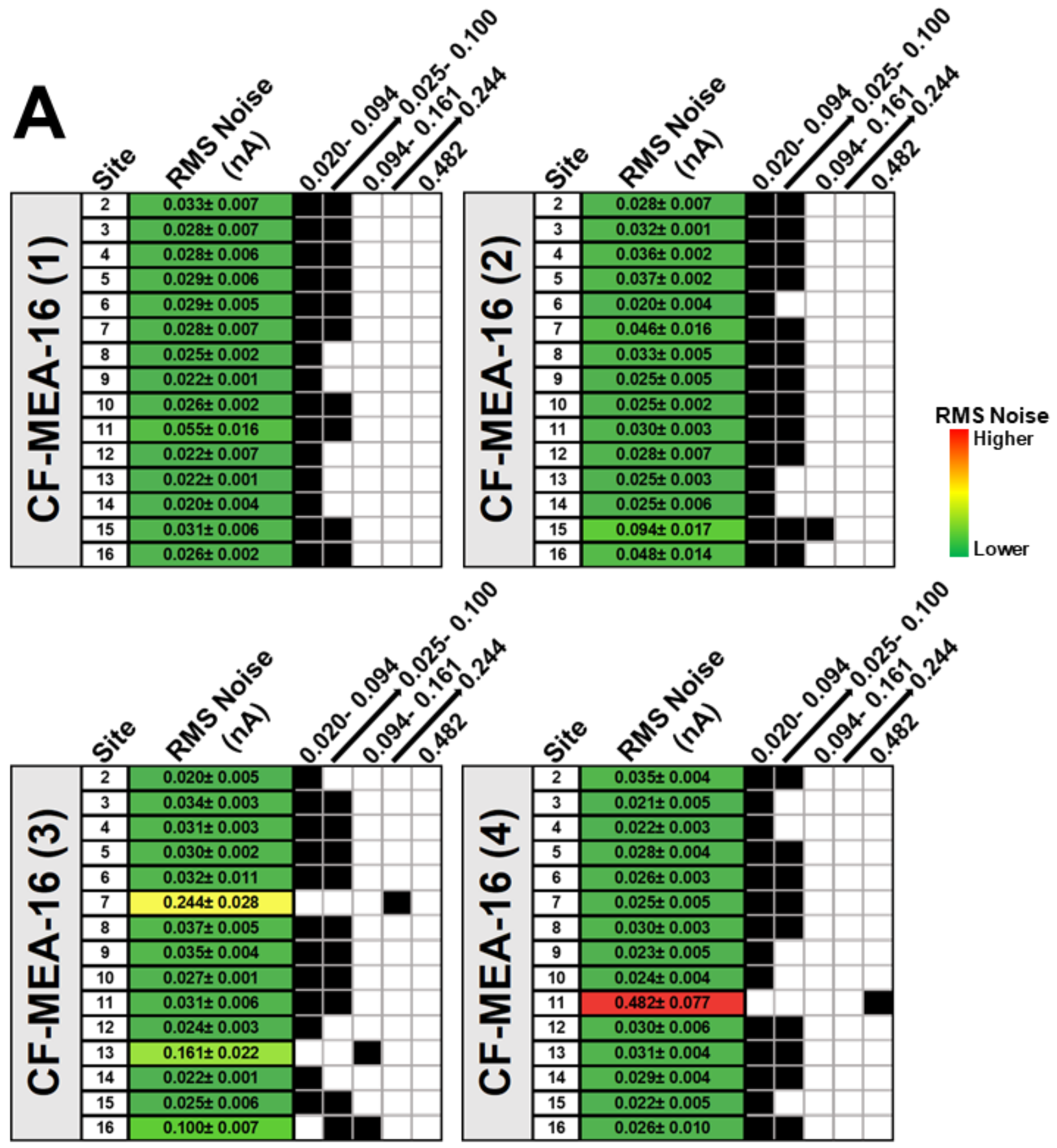




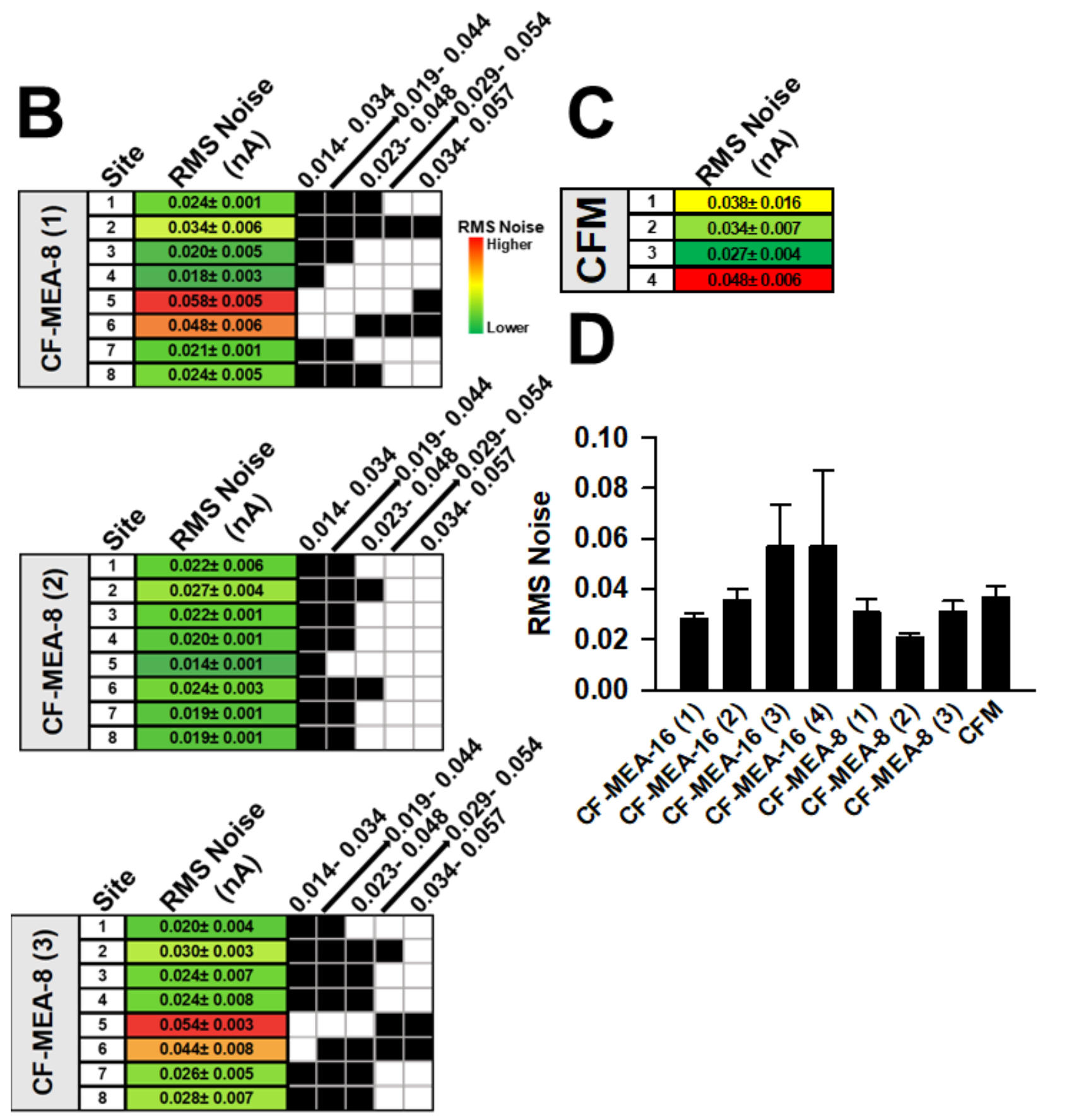

Figure 12. Statistical analysis of RMS noise. A nested one-way ANOVA was performed on RMS noise for CF-MEA-16s (A) and CF-MEA-8s (B), and a one-way ANOVA was performed on RMS noise for CFMs (C). A total of three replicates were analyzed for each site or CFM; data are mean \pm SEM of these replicates. No significant effect of CFM on RMS noise was found. Post- 
hoc Tukey-Kramer analysis is depicted by statistical groupings as in Figure 7. Color scale represents sensitivity relative to each sensor type. A one-way ANOVA was performed on RMS noise across CF-MEA-16s, CF-MEA-8s, and CFMs (D). Data are mean \pm SEM of each site of the arrays or CFM after replicate averaging. No significant effect of sensor on RMS noise was found. 
Figure 13: Statistical analysis of TBC
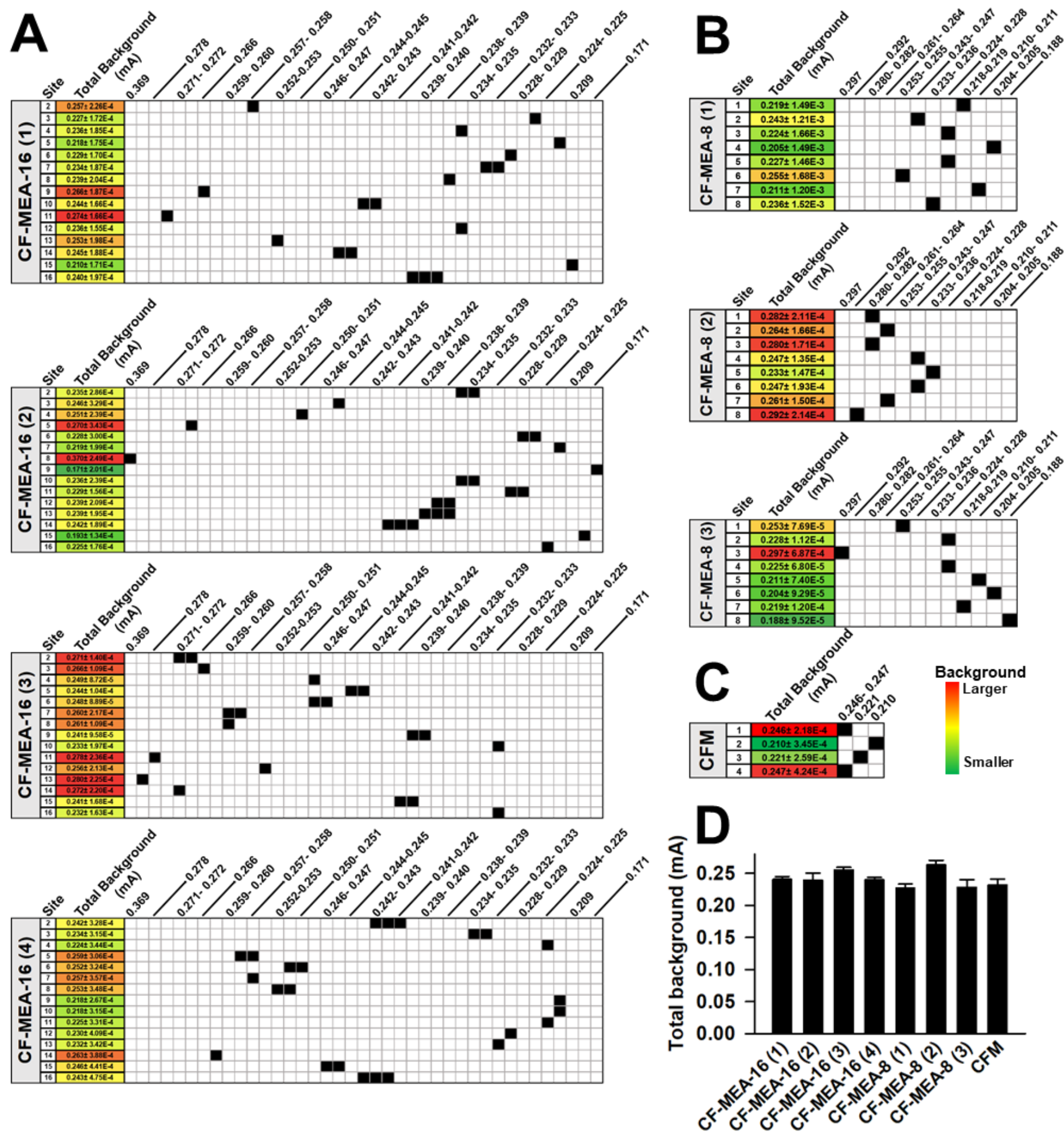
Figure 13: Statistical analysis of TBC. A nested one-way ANOVA was performed on RMS noise for CF-MEA-16s (A) and CF-MEA-8s (B), and a one-way ANOVA was performed on RMS noise for CFMs (C). A total of three replicates were analyzed for each site or CFM; data are mean \pm SEM of these replicates. Post-hoc Tukey-Kramer analysis is depicted by statistical groupings as in Figure 7. Color scale represents sensitivity relative to each sensor type. A one-way ANOVA was performed on TBC across CF-MEA-16s, CF-MEA-8s, and CFMs (D). Data are mean \pm SEM of each site of the arrays or CFM after replicate averaging. A significant effect of sensor on TBC was found, but post-hoc analysis failed to identify a significant pairwise difference. 
Figure 14: Statistical Analysis of SNR

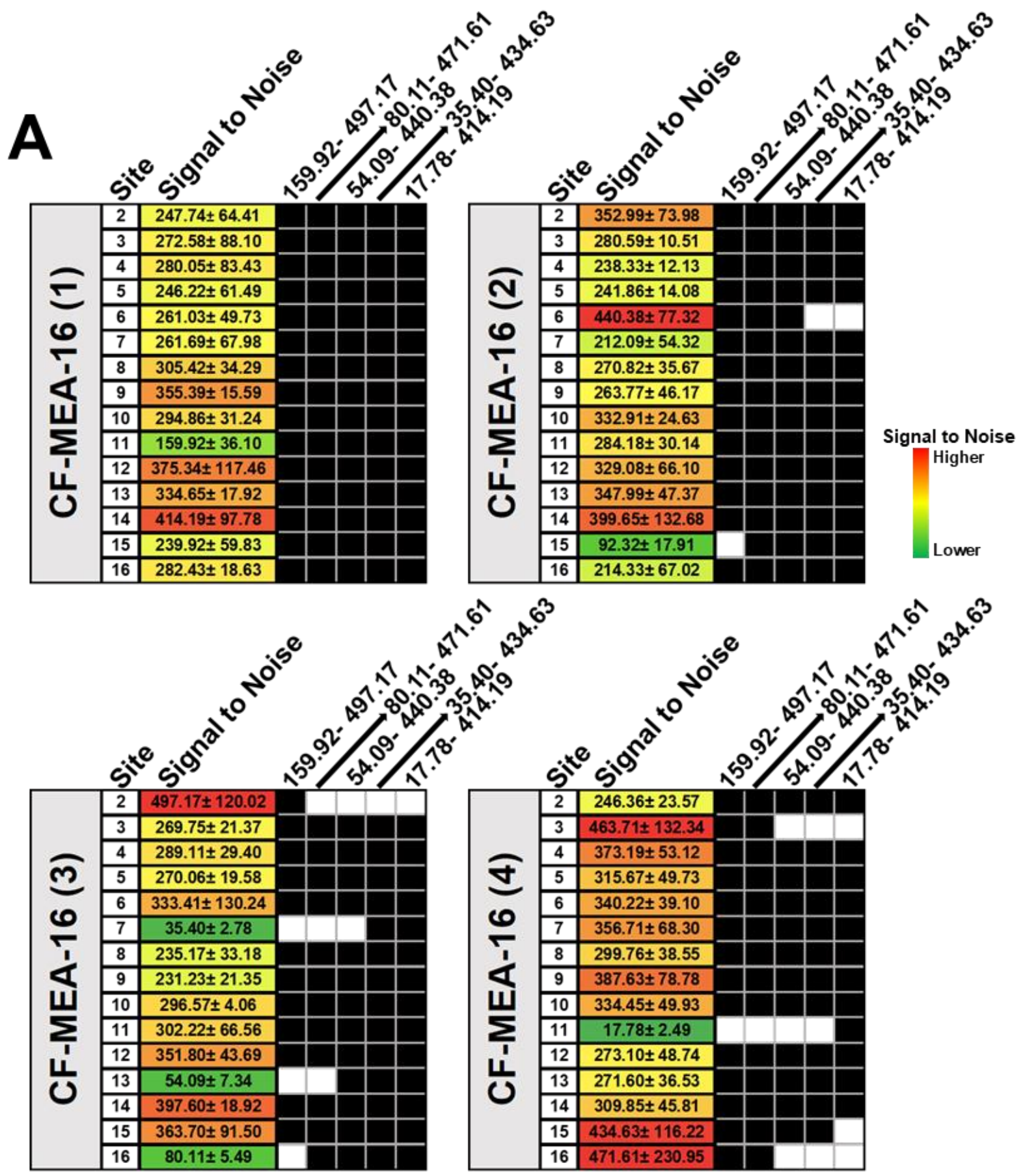



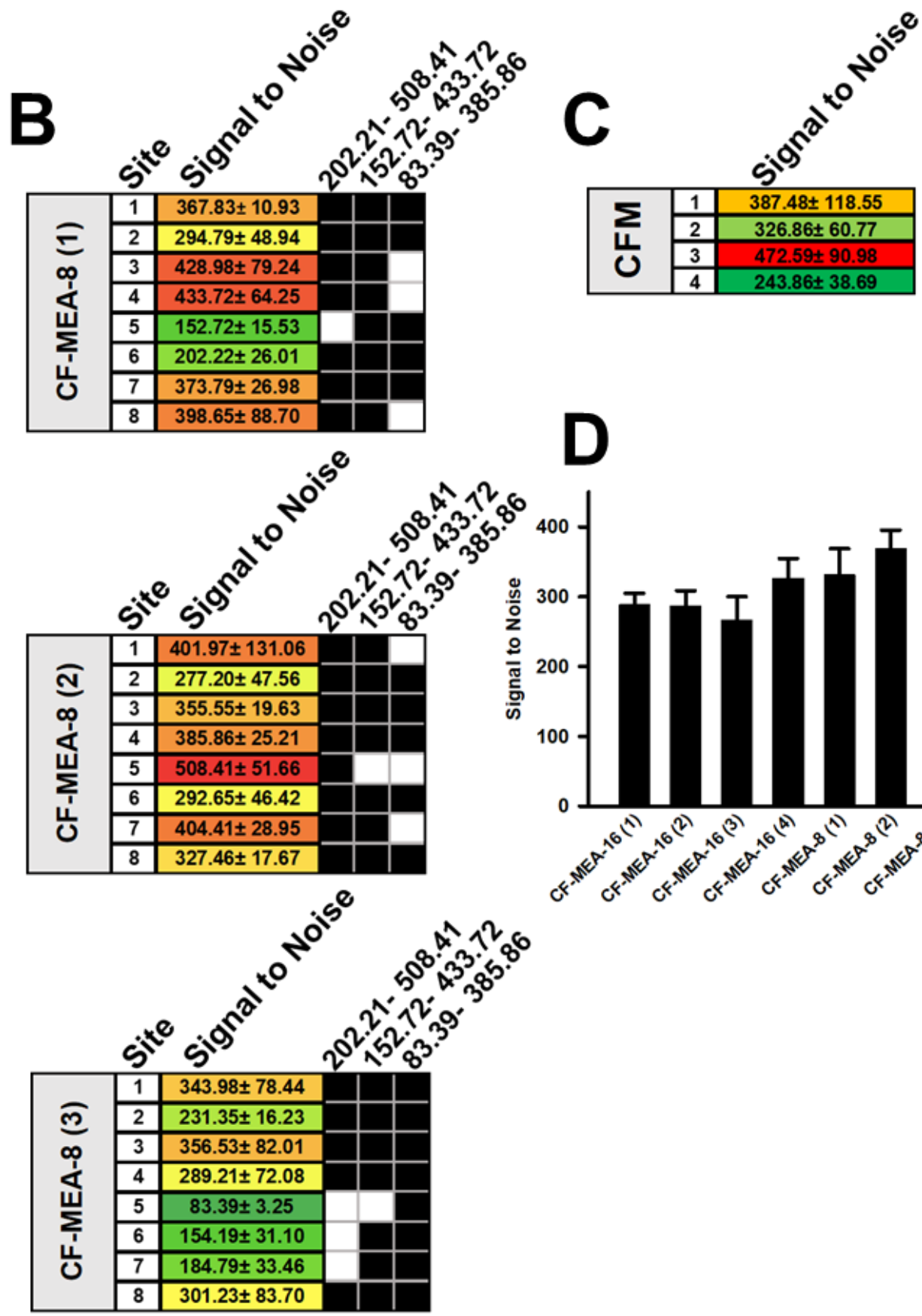

D

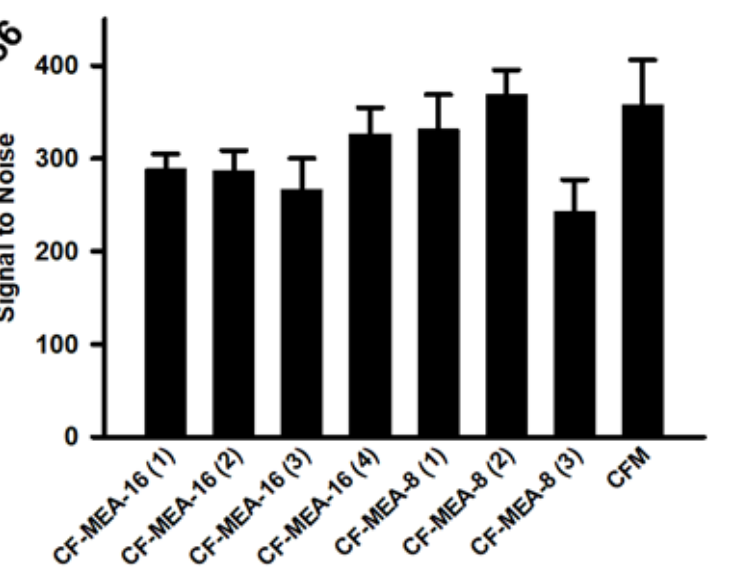

Figure 14: Statistical analysis of SNR. A nested one-way ANOVA was performed on RMS noise for CF-MEA-16s (A) and CF-MEA-8s (B), and a one-way ANOVA was performed on RMS noise for CFMs (C). A total of three replicates were analyzed for each site or CFM; data are mean \pm SEM of these replicates. No significant effect of CFM on RMS noise was found. Post-hoc Tukey- 
Kramer analysis is depicted by statistical groupings as in Figure 7. Color scale represents sensitivity relative to each sensor type. A one-way ANOVA was performed on SNR across CFMEA-16s, CF-MEA-8s, and CFMs (D). Data are mean \pm SEM of each site of the arrays or CFM after replicate averaging. No significant effect of sensor on SNR was found. 
Figure 15: PCR for resolving DA and $\triangle \mathrm{pH}$
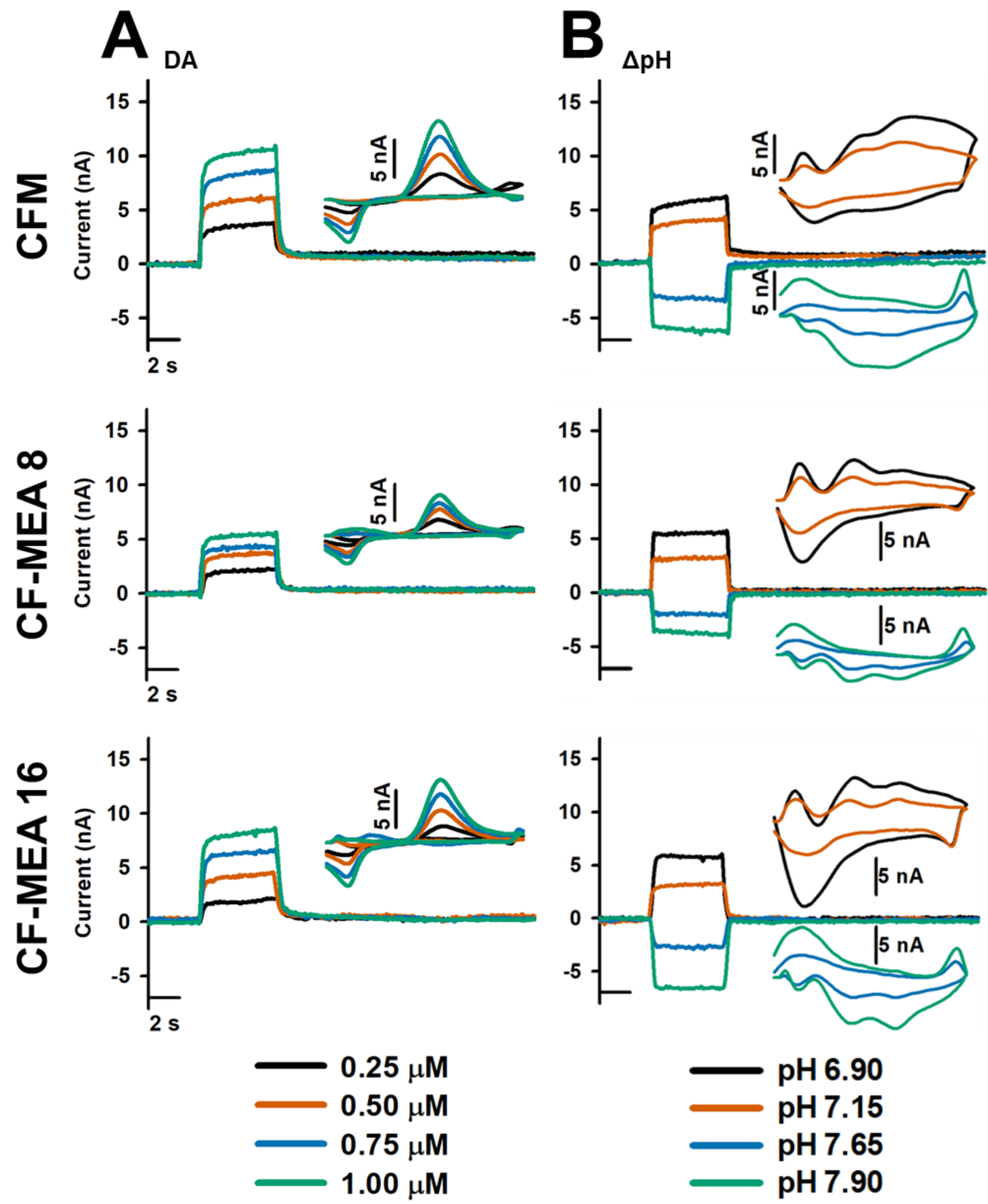

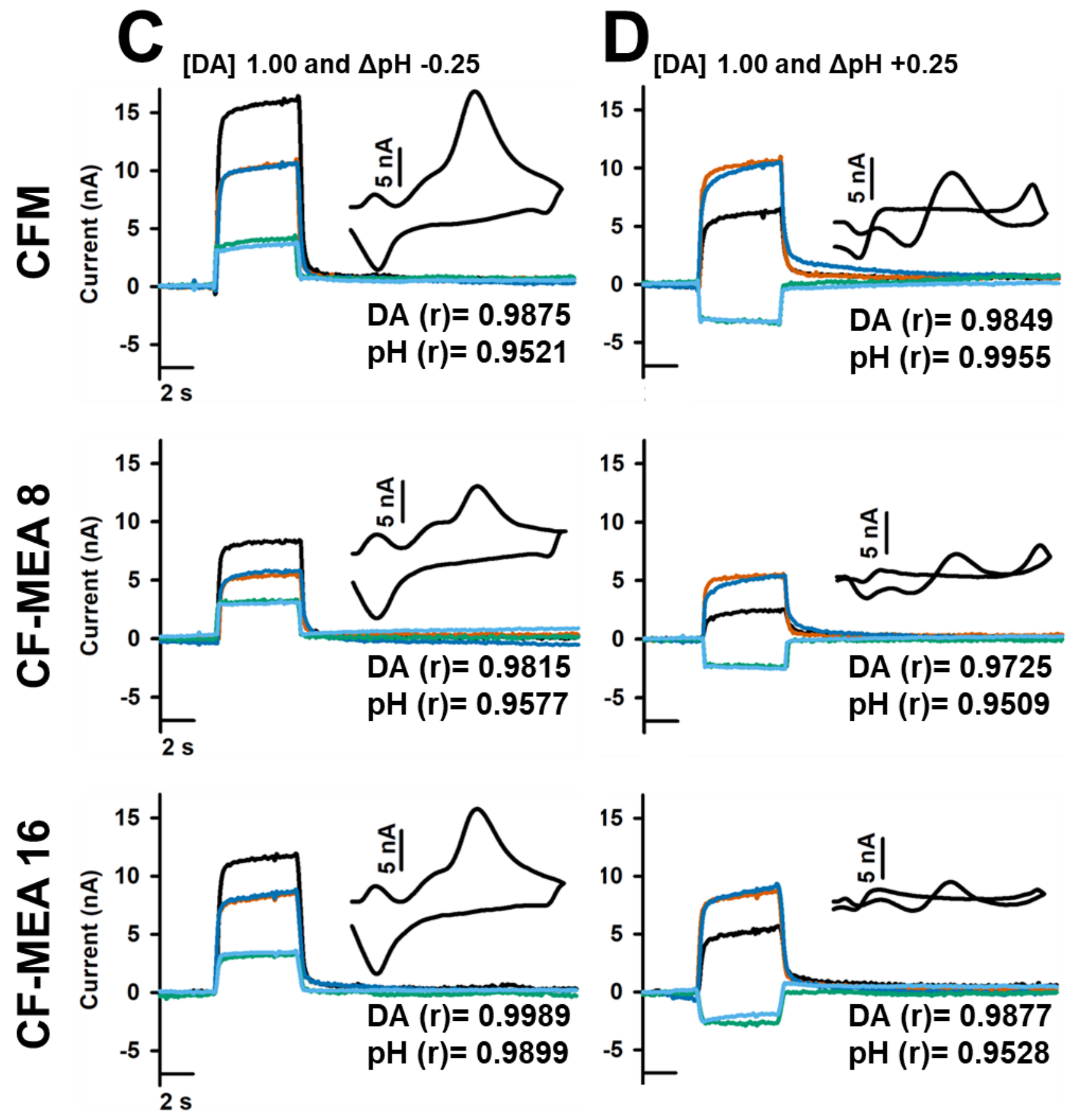

- Mixture [DA $1.00 \mu \mathrm{M}+\mathrm{pH} 7.15]$

Mixture [DA $1.00 \mu \mathrm{M}+\mathrm{pH} 7.65]$

DA [1.0 $\mu \mathrm{M}]$ Observed

PCR Resolved DA

$\mathrm{pH}$ [7.15] Observed

PCR Resolved pH

DA [1.0 $\mu \mathrm{M}]$ Observed

PCR Resolved DA

$\mathrm{pH}$ [7.65] Observed

PCR Resolved pH 
Figure 15: PCR for resolving DA and pH. Temporal responses for different concentrations of DA (A) and values for $\triangle \mathrm{pH}(\mathbf{B})$ and collected by FIA are shown for a CFM (top) and a one site of a CF-MEA-8 (middle) and CF-MEA-16 (bottom), respectively. Current for both DA and $\Delta \mathrm{pH}$ was measured at the peak oxidative potential for DA. INSETS are background-subtracted cyclic voltammograms. The DA concentrations are $0.25,0.50,0.75$, and $1.0 \mu \mathrm{M}$, and $\Delta \mathrm{pH}$ values are $0.50,-0.25,+0.25$, and +0.50 . The $\mathrm{pH}$ of the calibration buffer flowing across the sensor prior to analyte injection in FIA is 7.4. All DA solutions were also at a pH of 7.4. The voltammograms and calibrations create the training sets for PCR. C and $\mathbf{D}$ show PCR of two different mixtures of DA and $\Delta \mathrm{pH}, 1-\mu \mathrm{M}$ DA and a $\Delta \mathrm{pH}$ of -0.25 and $1-\mu \mathrm{m}$ DA and a $\Delta \mathrm{pH}$ of +0.25 , respectively, for the same CFM (top) and same single site of a CF-MEA-8 (middle) and CF-MEA-16 (bottom). The mixtures were selected because the acidic $\mathrm{pH}$ adds to the $\mathrm{DA}$ temporal response and the basic $\mathrm{pH}$ subtracts from the DA temporal response, thus providing a good test of the PCR analysis. Temporal responses and voltammograms (INSETS) of the mixtures are shown by black lines. The distortion of DA monitoring by $\Delta \mathrm{pH}$ is clearly evident in the temporal response and voltammogram. The corresponding DA and $\mathrm{pH}$ temporal responses from the training set ("observed") are shown by orange and green lines, respectively. Individual temporal responses for DA and $\Delta \mathrm{pH}$ resolved by the PCR ("predicted") are shown by blue and aqua lines. Pearson correlation coefficients (r) listed in each panel describe agreement between predicated and observed recordings during the 5-s analyte bolus. All coefficients were significant. 
Figure 16: Heterogeneity of 24-p electrically evoked DA signals in the striatum
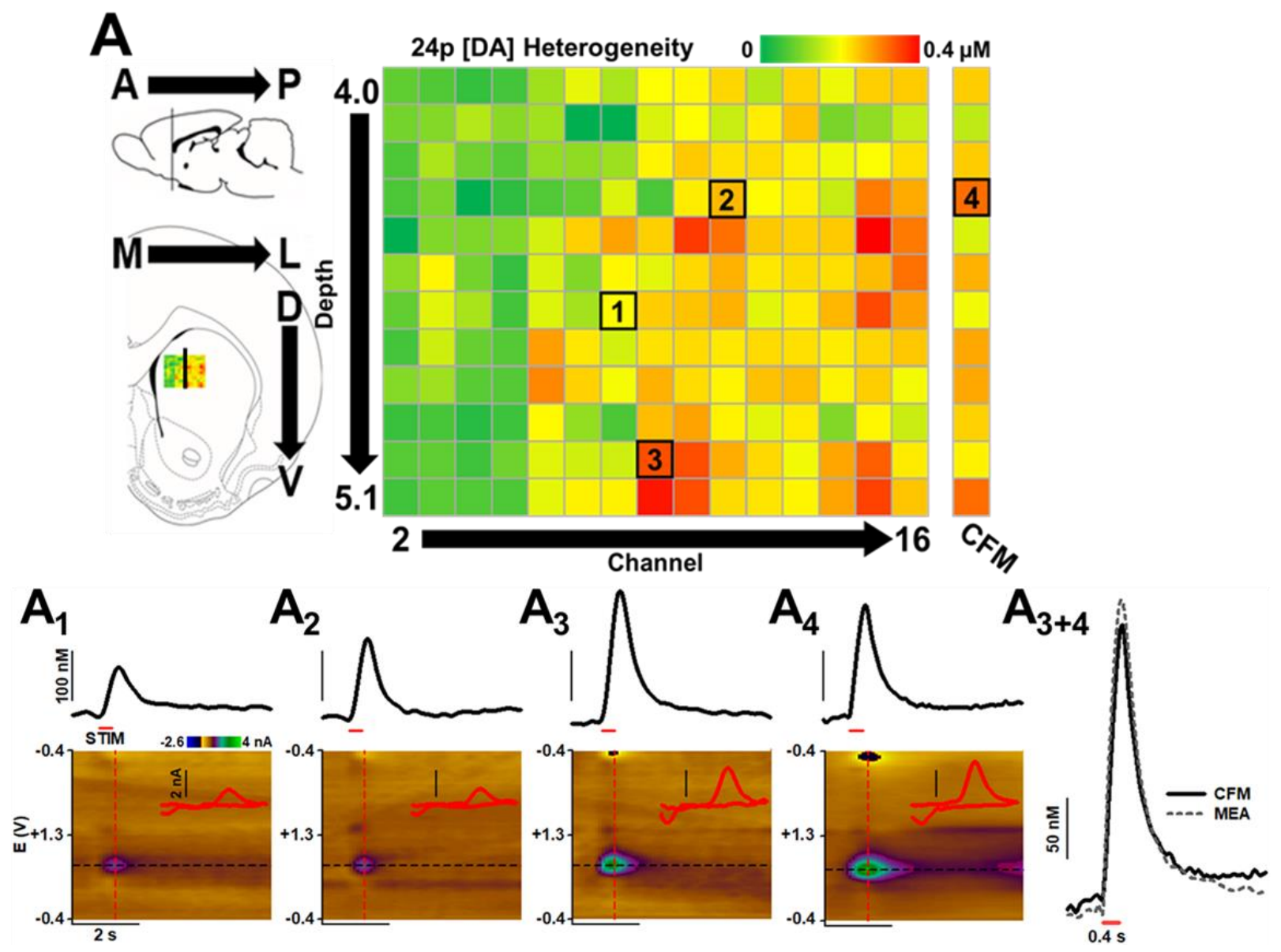

Figure 16. Heterogeneity of electrically evoked 24-p DA signals in the striatum. A. Transientlike DA signals electrically evoked by 0.4-s (24-pulse) trains were measured in the striatum of the urethane-anesthetized rat by a CFM or CF-MEA-16 lowered in $100-\mu \mathrm{m}$ increments. After collecting recordings, the CFM was removed and the CF-MEA-16 was implanted for recordings. The mediolateral location of the CFM was approximately in the middle of the array. The anteroposterior and dorsoventral locations of the CFM and CF-MEA-16 were also approximately similar. Heat map (top) delineates [DA $]_{\max }$ at recording sites and dorsoventral positions. Temporal responses and associated voltammograms are shown for select locations in $\mathbf{A} \mathbf{1}$ to $\mathbf{A} 4$, designated 
by the corresponding number in the heat maps. Pseudocolor plots and individual voltammograms are consistent with DA as the electrically evoked analyte. A3+4 overlays the recordings at location 3 of the CF-MEA-16 and location 4 of the CFM. The approximate anteroposterior, mediolateral, and dorsoventral location of the CF-MEA-16 is shown in top left (Paxinos and Watson, 1986). Hardware issues prevented recording at site 1 of the CF-MEA-16. Numbers and letter correspond to location in the heat map. Horizontal lines underneath temporal responses demarcate the applied stimulus train. 
Figure 17: Heterogeneity of electrically evoked 180-p DA signals in the striatum

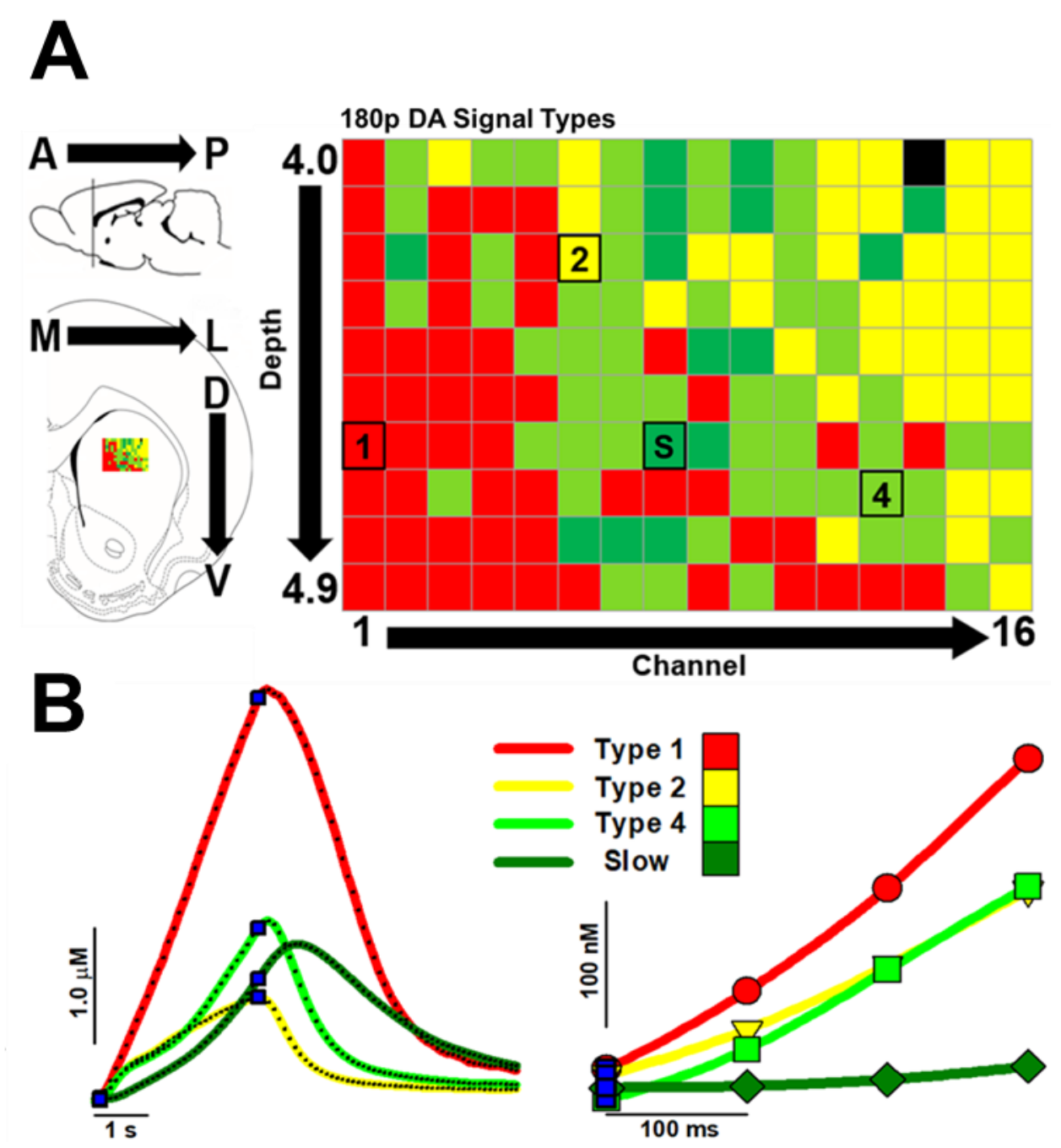

Figure 17: Heterogeneity of electrically evoked 180-p DA signals in the striatum. A. DA signals elicited by a 3-s (180-pulse) train were categorized into different types (Taylor et al., 2015). Type 1 signals increase linearly during the pulse train. Type 2 signals also increase but not linearly. No type 3 signals, for which the maximum is reached before the end of the stimulus train, were recorded. Type 4 signals are biphasic. Slow signals exhibit an initial delay or lag in the DA increase at the beginning of the pulse train. See Fig. 18B for $[D A]_{\max }$ elicited by the 3 -s pulse trains. The approximate anteroposterior, mediolateral, and dorsoventral location of the CF-MEA-16 is shown 
at left. B. Representative recordings of the fast and slow types of evoked DA signals (left) are shown with their initial increases expanded in time (right). Numbers and letter correspond to location in the heat map. Blue boxes in temporal responses indicate stimulation on at 0-s (left and right) and off at 3-s (left). 
Figure 18: Comparison of DA signals elicited by 0.4 and 3-s pulse trains

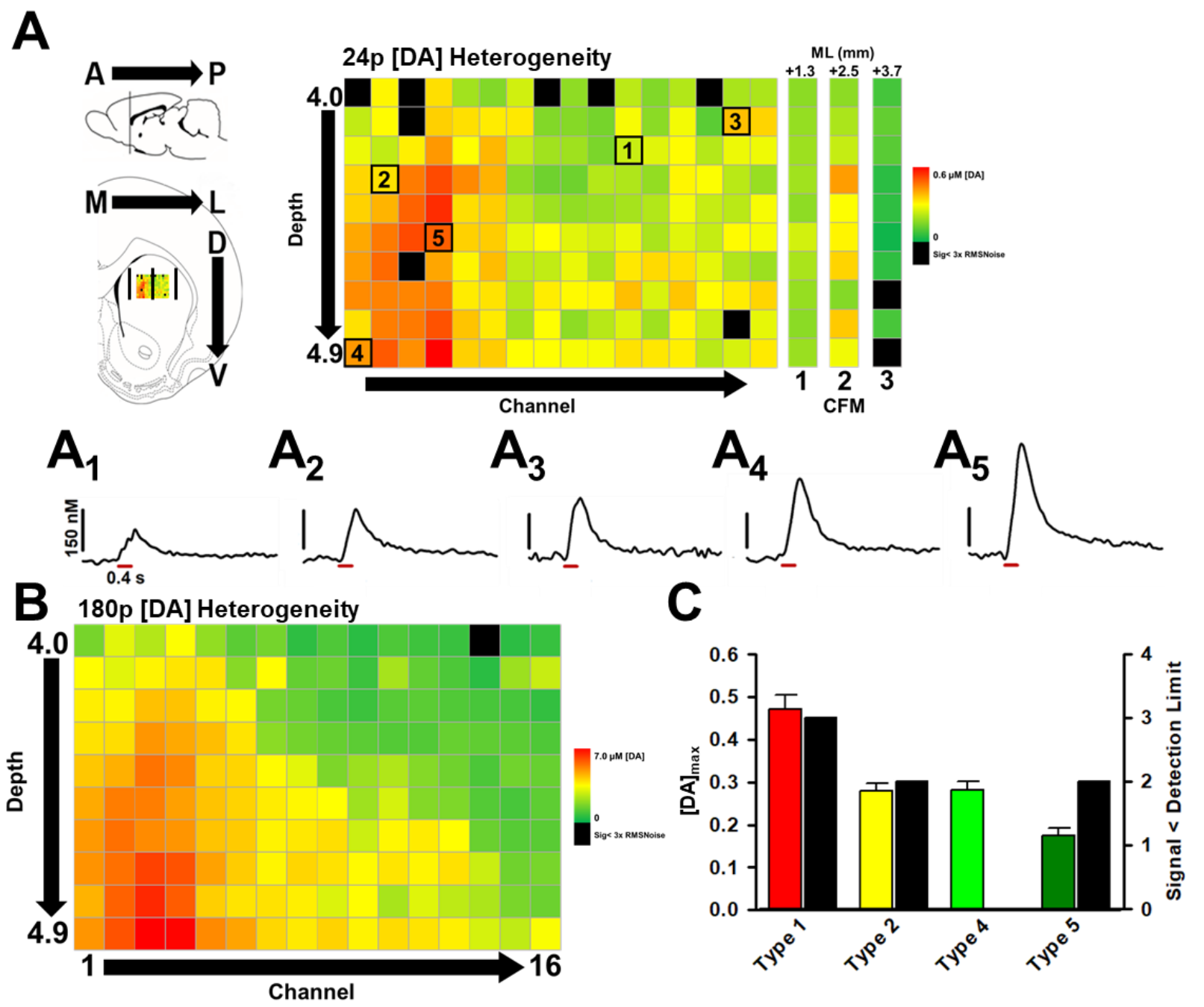

Figure 18. Comparison of DA signals elicited by 0.4- and 3-s pulse trains. A CF-MEA-16 was implanted in the lateral aspect of the dorsomedial striatum of the anesthetized rat to record DA signals electrically evoked by 0.4-s, 24-pulse and 3-s,180-pulse trains (A and B, respectively). Calculated $[\mathrm{DA}]_{\max }$ is plotted as heat maps. Underneath the heat map for DA signals elicited by 0.4-s trains (A) are representative temporal responses and associated voltammograms for select sites designated $\mathbf{A}_{\mathbf{1}}$ to $\mathbf{A} \mathbf{5}$, corresponding to numbered sites in the heat map. Horizontal red lines demarcate the stimulus train. Recordings analyzed for $\mathbf{B}$ were used to identify the type of DA 
signal described in Figure 17. $\mathbf{C}$ shows $[\mathrm{DA}]_{\max }$ (left y axis) and the number of sites without detectable DA when elicited by a 0.4-s train (right y axis) for each type of DA signal. [DA $]_{\max }$ is expressed as the mean \pm SEM. Signal type is color coded according to Figure 17. 
Figure 19: Sensor Stability and Dynamic Range

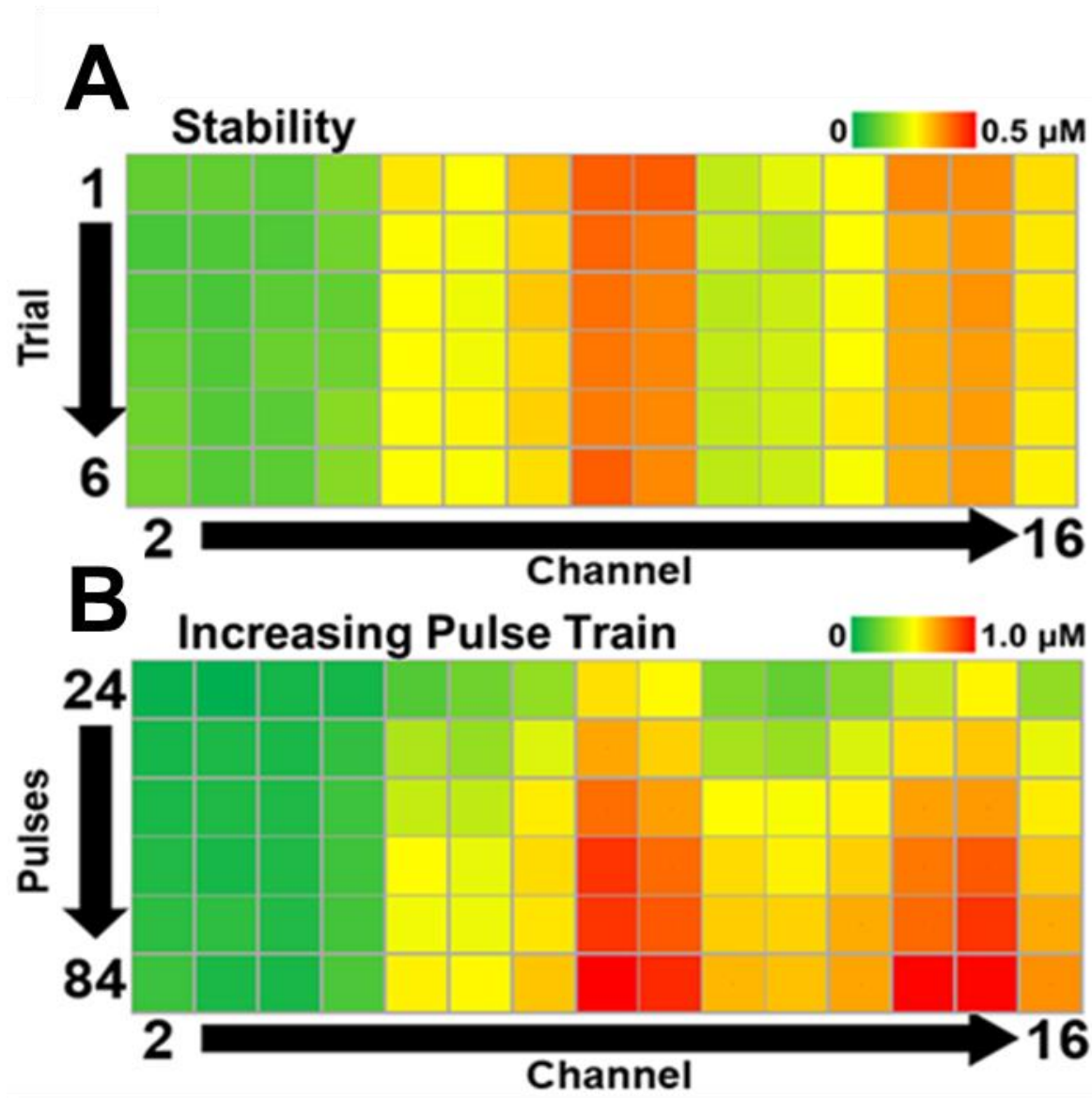

Figure 19: Sensor Stability and Dynamic Range. A. [DA $]_{\max }$ elicited by a $0.4-\mathrm{s}$ train and recorded by the CF-MEA-16 at a fixed location is stable over time. Trials were separated by 5 min. B. $[\mathrm{DA}]_{\max }$ increases with the number of stimulus pulses. Stimulus trains consisted of $24,36,48$, 60, 72, and 84 pulses, and measurements were collected at a fixed location of the CF-MEA-16. Hardware issues prevented recording at site 1 of the CF-MEA-16. 
Figure 20: Comparison of in vivo SNR for monitoring DA signals
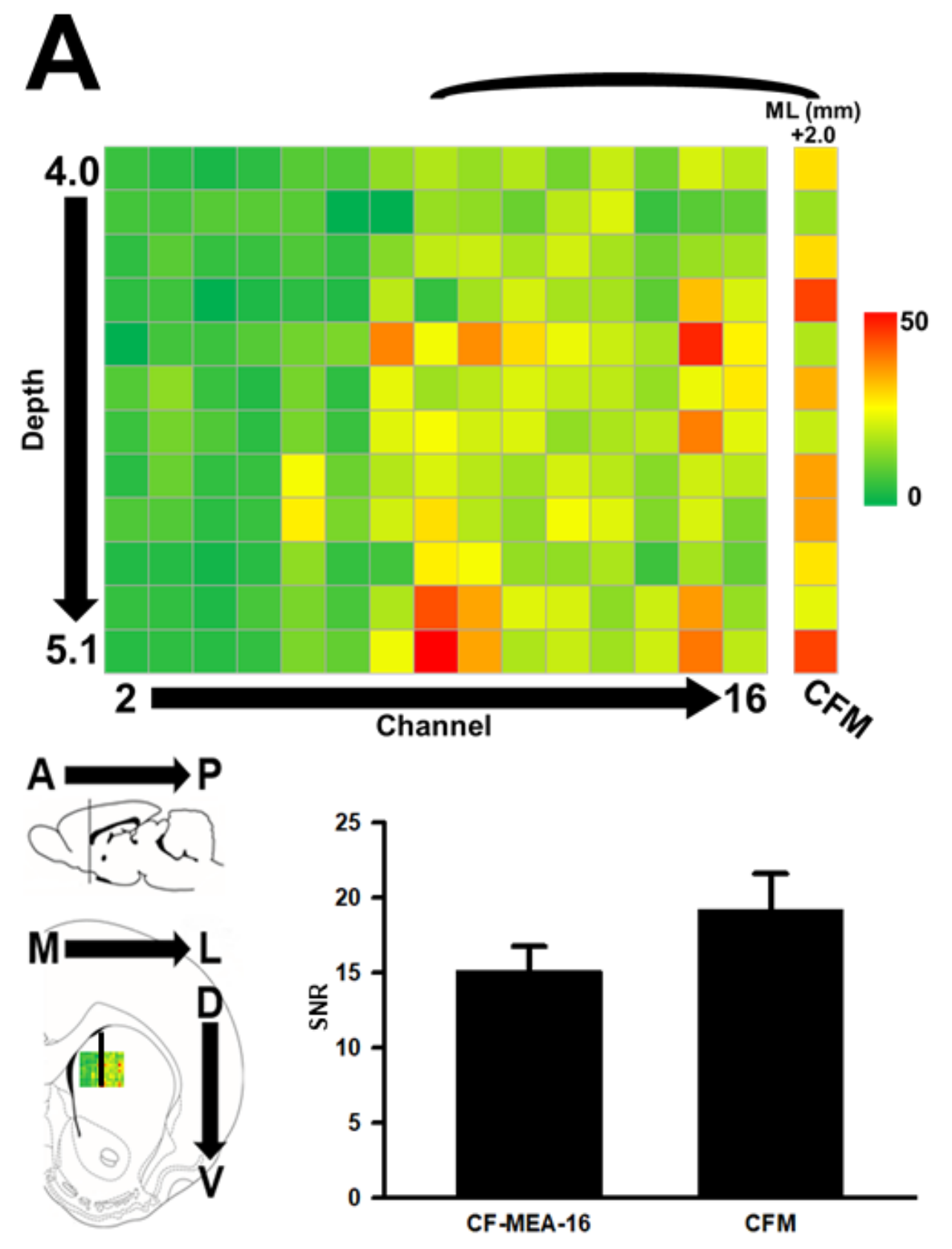


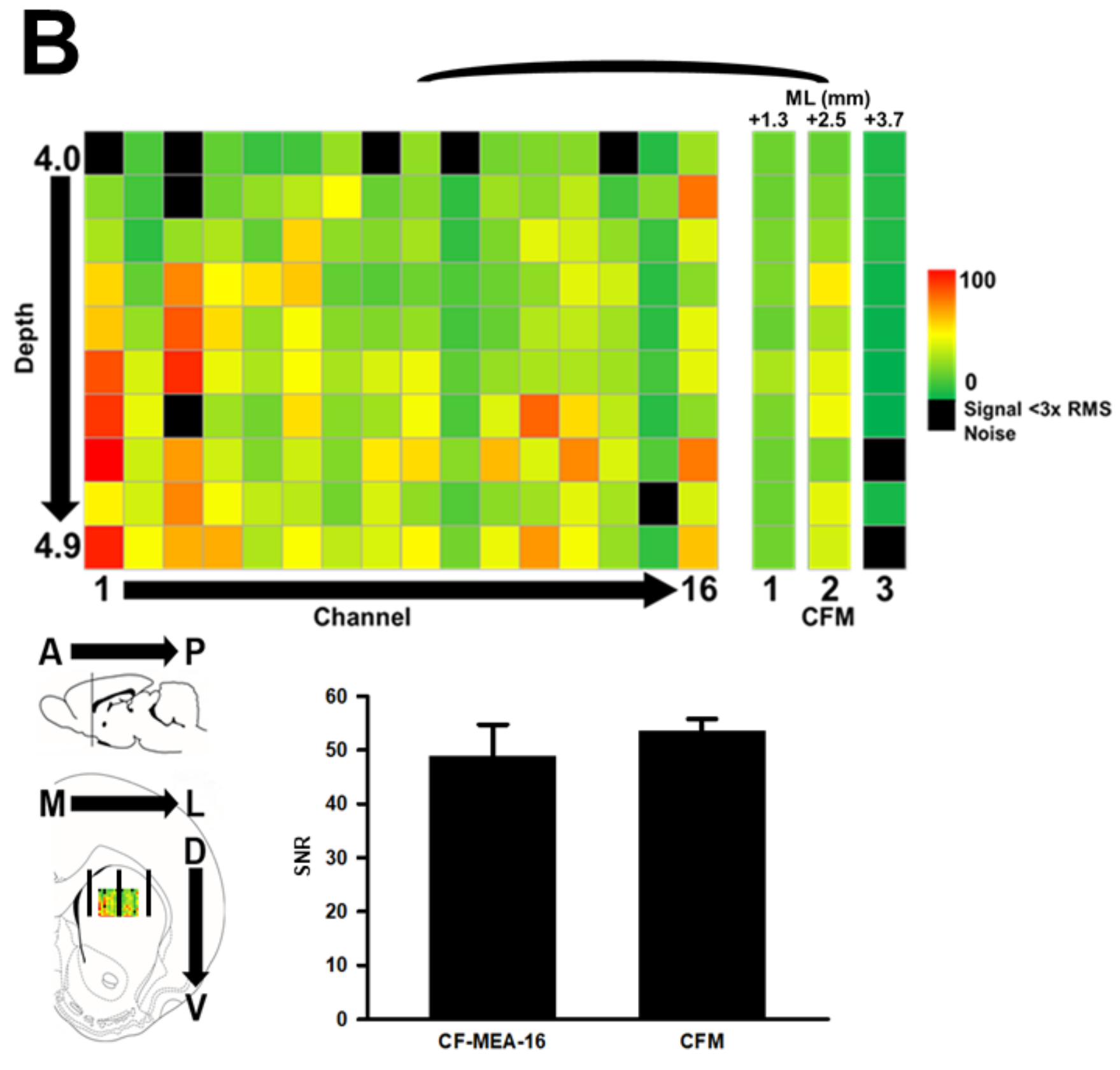

Figure 20: Comparison of in vivo SNR for monitoring DA signals. SNR was determined for the electrically evoked DA signals shown in Figures 16A and 18A and plotted as heat maps (A. and B. top, respectively). Hardware issues system prevented recording at site 1 of the CF-MEA16 in A. Sensors of different types were not implanted at the same time; thus, CFM and array 
recordings were not simultaneously recorded. In A, the CFM was implanted first, measured DA, and then withdrawn. The array was then implanted to approximately the same dorsoventral, mediolateral, and anteroposterior position of the CFM. Positioning was similar in B, except that three CFMs were simultaneously implanted. The approximate mediolateral location of the CFM in $\mathbf{A}$ is the middle of the CF-MEA-16 at sites 7 to 9 (top and bottom left). The approximate mediolateral location of the second CFM in $\mathbf{B}$ is the middle of the CF-MEA-16 at sites 9 to 10 (top and bottom left). The first and third CFMs were located $1.2 \mathrm{~mm}$ mediolaterally to either side of the second CFM and recorded DA signals in striatal subregions spatially beyond monitoring by the CF-MEA-16 (bottom left). Recordings at all three CFM were collected simultaneously. The bar graph at bottom right compares SNR for the CFM in A and middle CFM in $\mathbf{B}$ with the three middle sites of each respective CF-MEA-16. SNR was averaged across dorsoventral position for both CFM and CF-MEA-16. However, SNR was first averaged across the three middle sites of each array canvassing the approximate mediolateral location of the CFM. T-tests failed to find a significant difference in SNR between the array and CFM in $\mathbf{A}\left(\mathrm{t}_{22}=1.378 ; \mathrm{p}=0.18\right)$ and $\mathbf{B}\left(\mathrm{t}_{18}=\right.$ $0.7413 ; \mathrm{p}=0.47)$. 
Figure 21: AMPH modulated electrically evoked transient-like DA signals in the striatum

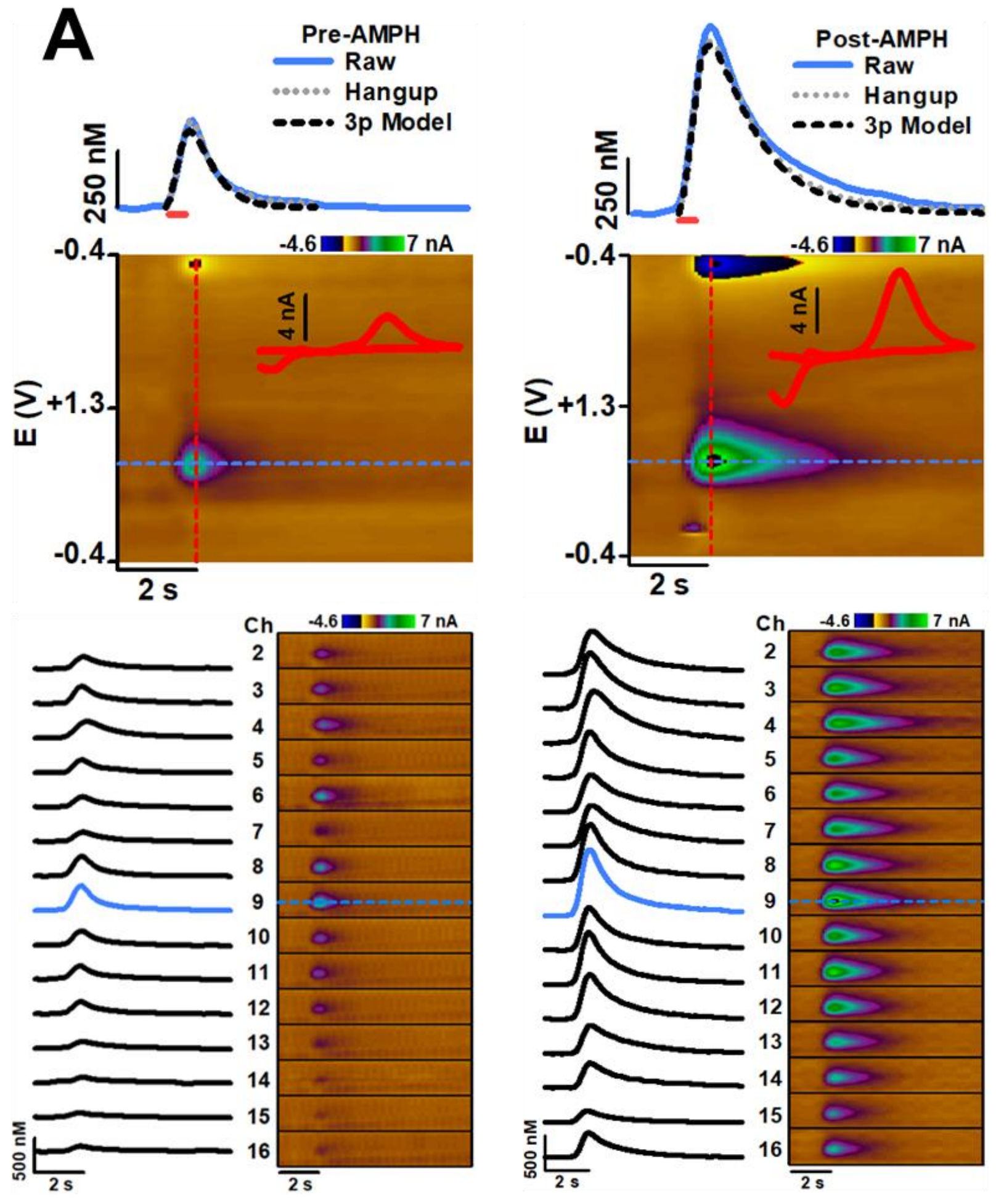




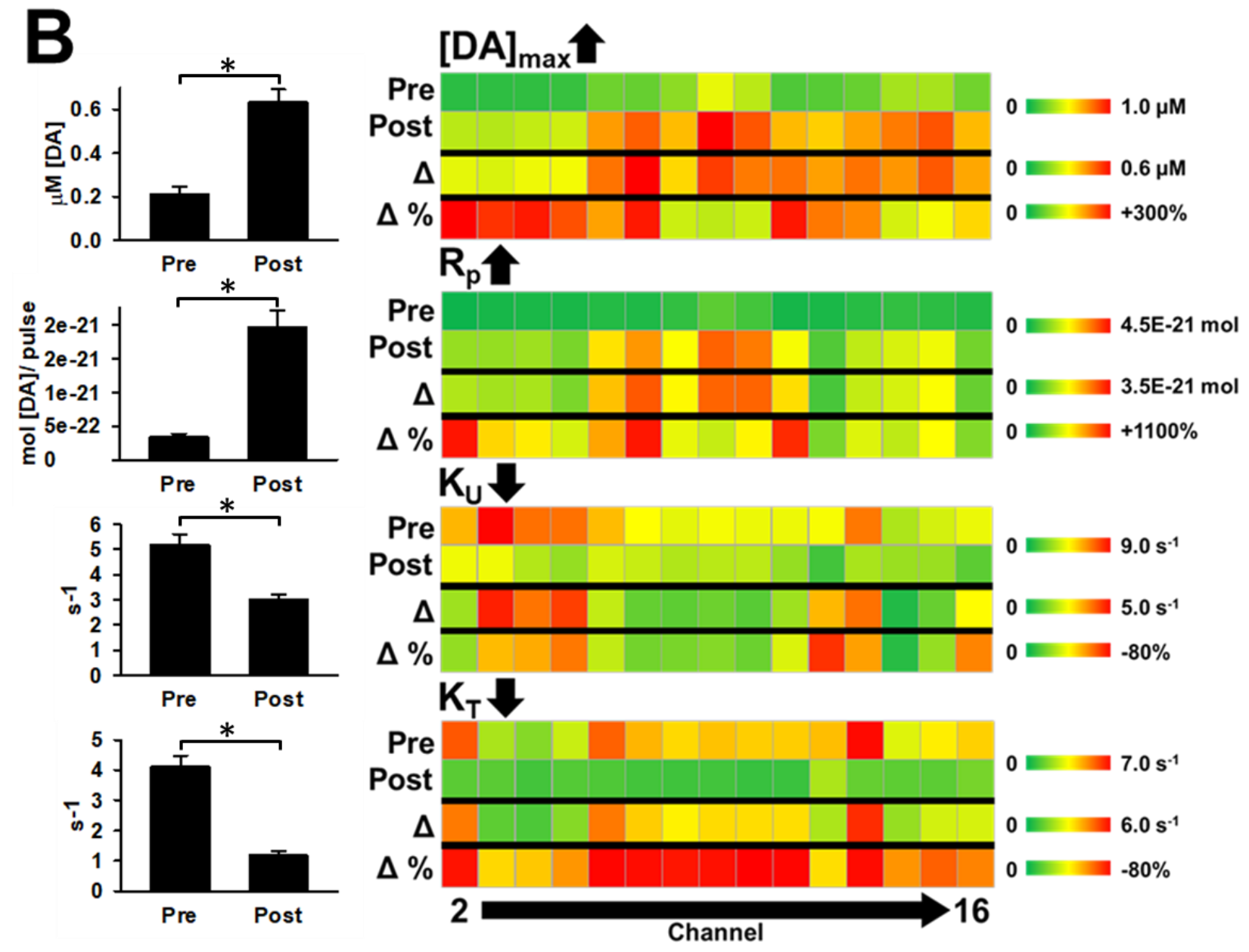

Figure 21. AMPH modulates electrically evoked transient-like DA signals in the striatum. A.

Transient-like DA signals electrically evoked by a 0.4-s train were measured in the striatum of the urethane-anesthetized rat by a CF-MEA-16. The location of these recordings is the lowest dorsoventral position in Figure 2A. Pre- and post-AMPH recordings are shown to left and right, respectively. Top temporal responses are the raw FSCV recording (solid blue line), raw FSCV recording with the hang-up removed (gray dotted line), and the simulated response calculated from parameters derived from model fitting of the recording with hang-up removed (dashed black line). Horizontal red lines underneath temporal responses demarcate the applied stimulus train. The raw FSCV recording is current measured at the peak oxidative potential for DA (horizontal blue line 
in pseudocolor plot underneath) and converted to concentration. See Figure 1 for details of the pseudocolor plot. The hang-up removed from the raw FSCV response is attributed to DA adsorption to the carbon fiber (Walters et al., 2015). Bottom recordings show simultaneously recorded temporal responses and the associated portion of the pseudocolor plot for the applied potential $(+0.28$ to $0.96 \mathrm{~V})$ canvasing DA oxidation for pre (left)- and post (right)-AMPH conditions. Dashed lines represent current measured at the peak oxidative potential for DA, which is then converted to concentration for temporal responses. All recordings for site 9 are in blue. Hardware issues prevented recording at site 1 of the CF-MEA-16. B. Heat maps show, from top to bottom, [DA $]_{\max }, \mathrm{R}_{\mathrm{p}}, \mathrm{k}_{\mathrm{U}}$, and $\mathrm{k}_{\mathrm{T}}$ for pre- and post-AMPH conditions. $\Delta$ and $\Delta \%$ are absolute and percentage change, respectively, from pre- to post-AMPH recordings. 
Figure 22: AMPH elicits tonic and phasic DA signals
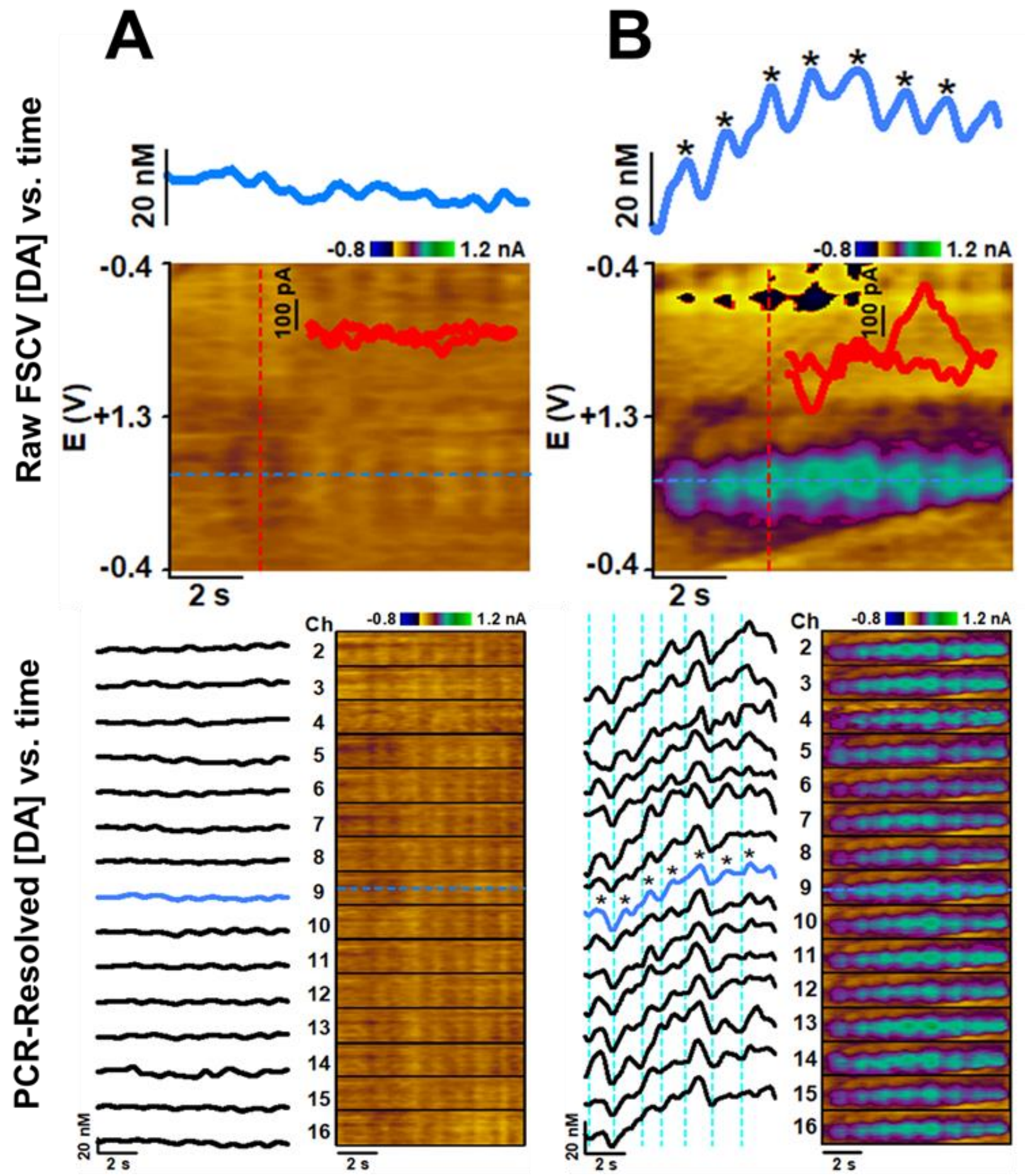

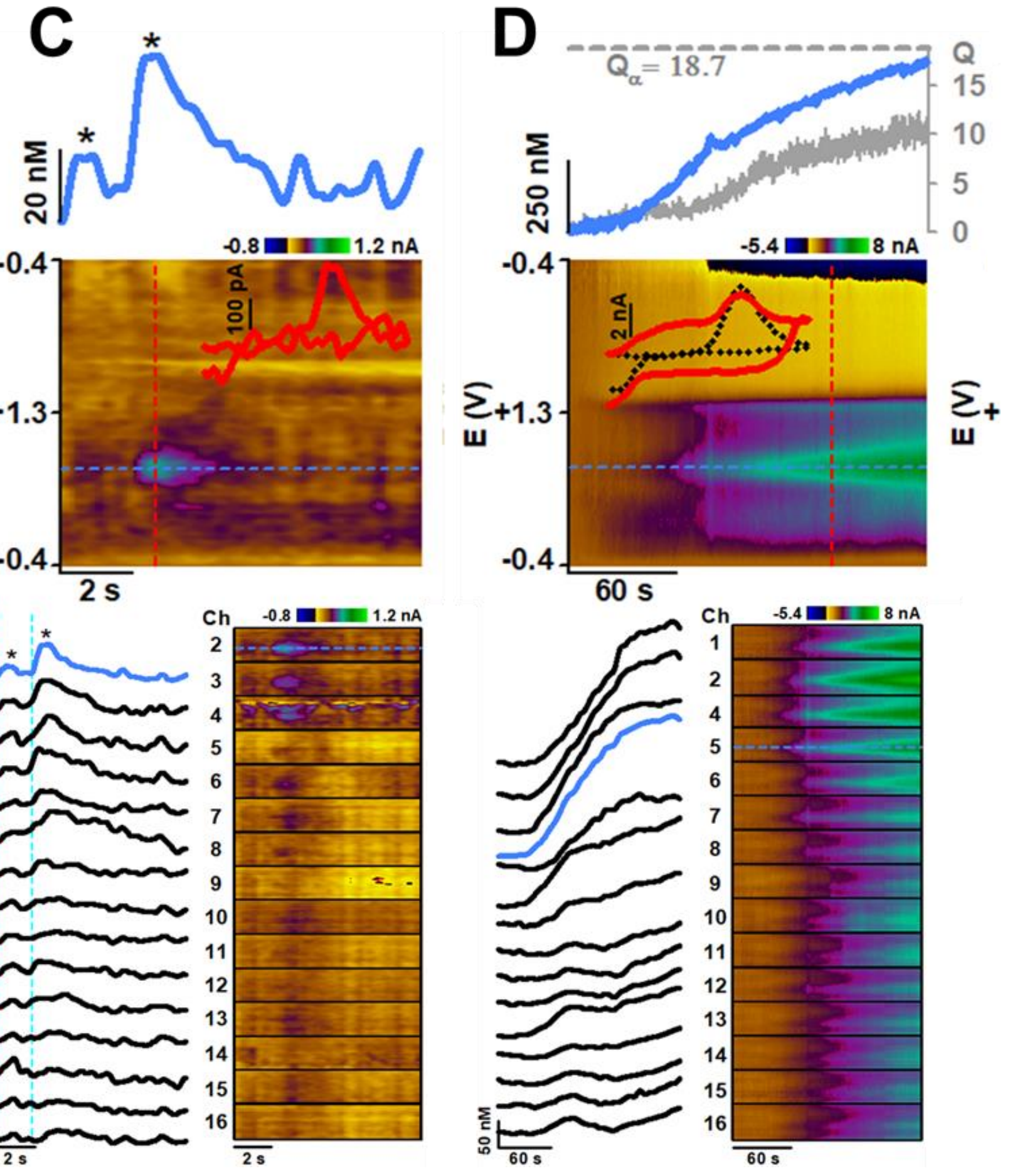


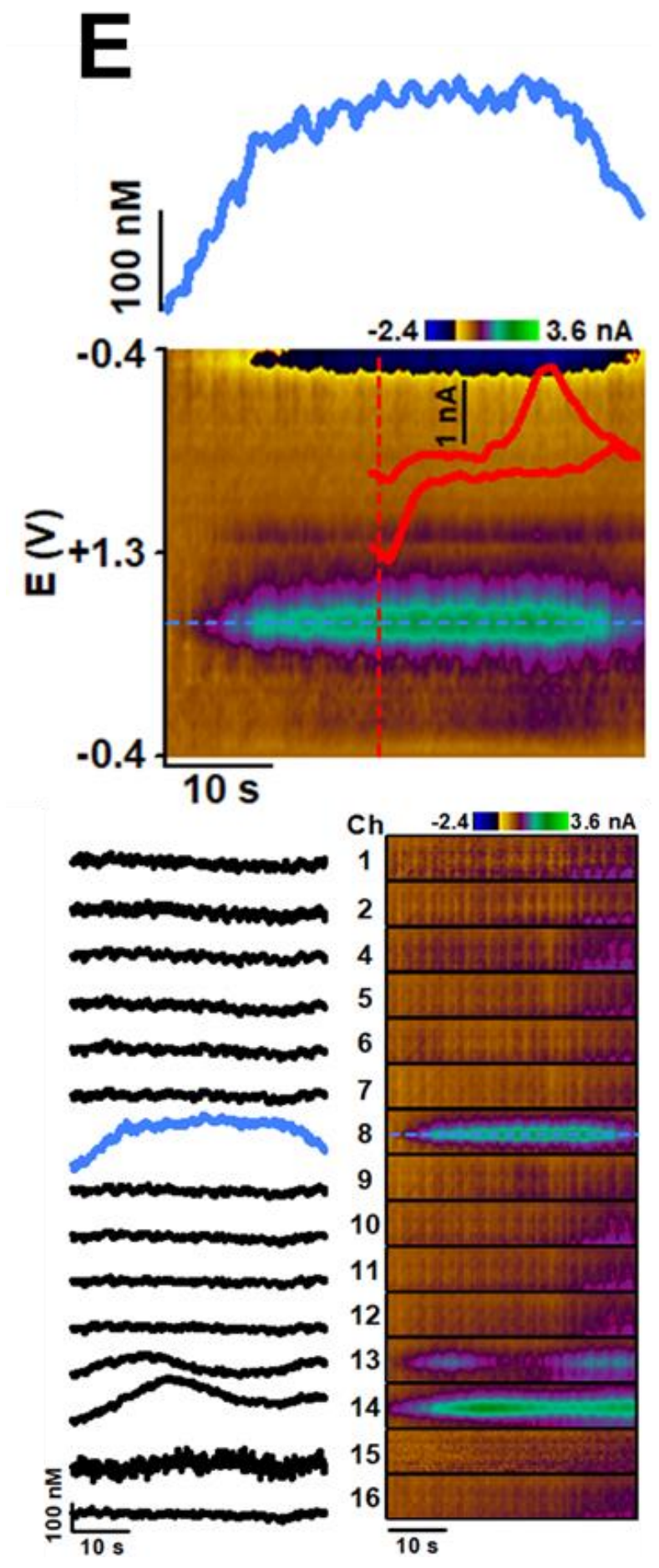

Figure 22. AMPH elicits tonic and phasic DA signals. Non-electrically evoked DA signals are shown for baseline (pre-drug) (A), post-AMPH (B and C), and post-AMPH and raclopride (D and E). Top shows raw FSCV recordings converted to DA concentration and the corresponding 
pseudocolor plots and individual background-subtracted cyclic voltammograms underneath. See Figure. 1 for details. Bottom shows simultaneously recorded, PCR-resolved temporal responses for DA from 15 sites of the CF-MEA-16 (left) with the associated portions of the pseudocolor plot for the applied potential $(+0.28$ to $0.96 \mathrm{~V})$ canvasing DA oxidation (right). Blue lines identify recording sites for temporal responses show above (top). Asterisks denote DA transients identified by analysis of PCR-resolved recordings with peak-finding software. A. There is no evidence of changes in DA during these pre-drug recordings collected five minutes before AMPH administration. B. Recordings were collected three minutes after AMPH (1.0-mg/kg i.v.) administration. All 15 sites of the CF-MEA-16 simultaneously recorded DA transients. C. Recordings were collected six minutes after AMPH (1.0-mg/kg i.v.) administration. Not all sites of the CF-MEA-16 recorded DA transients. Hardware issues prevented recording at site 1 of the CF-MEA-16 in A, B, and C. D. Recordings were collected two minutes after i.p. administration of AMPH (10 mg/kg) and raclopride (2 mg/kg). All sites show an increase in DA baseline, although to varying degrees. Top plot of temporal response additionally shows the corresponding Q-values (gray line) for the PCR analysis of the raw FSCV recording. All Q-values were below $\mathrm{Q}_{\alpha}$ (18.7; dashed gray line), indicating a successful PCR. Top pseudocolor plot shows an additional background-subtracted cyclic voltammogram collected at this CF-MEA-16 site from an electrically evoked signal (dashed black line). This voltammogram serves as a DA reference to highlight the DA component of the raw FSCV recording. While DA peaks are evident in the voltammogram, other features are as well. These later interferents are removed by PCR to reveal the DA component. E. Recordings were collected 50 minutes after i.p. administration of AMPH $(10 \mathrm{mg} / \mathrm{kg})$ and raclopride $(2 \mathrm{mg} / \mathrm{kg})$. Not all sites show an increase in DA baseline. 